\title{
A MATHEMATICAL MODEL FOR THE INTRACELLULAR KINETICS OF INFLUENZA A VIRUS REPLICATION IN VITRO
}

by

Diana Schwendener Forkel

B.Sc. Ryerson University, Toronto (ON), Canada, 2014

\author{
A thesis \\ presented to Ryerson University \\ in partial fulfillment of the \\ requirements for the degree of \\ Master of Science \\ in the Program of \\ Molecular Science
}

Toronto, Ontario, Canada, 2016

(C) Diana Schwendener Forkel 2016 


\section{Author's Declaration}

I hereby declare that I am the sole author of this thesis. This is a true copy of the thesis, including any required final revisions, as accepted by my examiners.

I authorize Ryerson University to lend this thesis to other institutions or individuals for the purpose of scholarly research.

I further authorize Ryerson University to reproduce this thesis by photocopying or by other means, in total or in part, at the request of other institutions or individuals for the purpose of scholarly research.

I understand that my thesis may be made electronically available to the public for the purpose of scholarly research only. 


\begin{abstract}
A mathematical model for the intracellular kinetics of influenza $A$ virus replication in vitro

Diana Schwendener Forkel

Master of Science, Molecular Science

Ryerson University, 2016
\end{abstract}

In the last twenty years, mathematical modelling (MM) has been notably used to capture the infection kinetics of many infectious diseases as it allows insights into the basic biology, infection kinetics, and the mechanisms and efficacy of treatment modalities. MMs of influenza A virus (IAV) infection usually represent the process of virus replication within a cell as a 'black box' term for viral production. The simplification is appropriate when we are not interested in the microscopic details of infection. Recently though, MMs have begun to account for the kinetics of intracellular IAV replication. Herein, we examine the MM by Heldt et al., which is able to capture kinetics of IAV infection. It however, does so by adjusting parameters of the MM to various events in the infection process. We developed a robust, yet concise, MM for the intracellular kinetics of influenza A virus infection in vitro with a consistent set of parameters. We use attachment, fusion and RNA data gathered from literature sources to validate our simplified MM and match known infection kinetics consistent throughout infection. 


\section{Table of Contents}

Table of Contents $\quad$ i

Authors Declaration $\quad$ ii

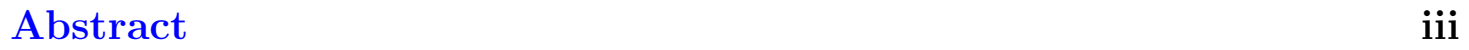

List of Tables $\quad$ vi

List of Figures $\quad$ vii

1 Introduction 1

1.1 Motivation . . . . . . . . . . . . . . . . . . 1

1.2 Goal . . . . . . . . . . . . . . . . . . . . . . . 5

1.3 Influenza A virus structure and replication process . . . . . . . . . 6 
2.1 Experimental data . . . . . . . . . . . . . . . . 21

2.2 Mathematical modelling . . . . . . . . . . . . . . 23

3 Results $\quad 35$

3.1 Kinetics of attachment of free virions . . . . . . . . . . . . 35

3.2 Kinetics of virion entry and fusion . . . . . . . . . . . . . . 44

3.3 Kinetics of replication from fusion to progeny release . . . . . . . . 48

3.4 Extracellular validation of the intracellular MM . . . . . . . . 57

3.5 Using the MM to ask key virological questions . . . . . . . . . . 62

3.5.1 Replication kinetics under super-infection . . . . . . . . . . 62

3.5.2 Efficacy of different antiviral targets . . . . . . . . . . . . 67

4 Discussion 


\section{List of Tables}

2.1 Summary of Heldt et al.'s variable naming and description. . . . . . 28

2.2 Summary of Heldt et al.'s parameter naming and description. . . . 29

2.3 Summary of Heldt et al.'s parameter naming and description (continuation) ...................... 30

2.4 Summary of our variable naming and description. . . . . . . . . . 33

2.5 Summary of our degradation rates. . . . . . . . . . . . . 33

2.6 Summary of our best fit parameters. . . . . . . . . . . . 34

3.1 Best fit parameters for attachment. . . . . . . . . . . . . 41 


\section{List of Figures}

1.1 Reported annual Canadian hospitalizations. . . . . . . . . . . . 2

1.2 Mathematical model for influenza viral infection in vitro . . . . . . 4

1.3 Influenza A virus. . . . . . . . . . . . . . . . . . . 7

1.4 vRNP structure. . . . . . . . . . . . . . . . . . 8

1.5 Differences in the mechanism of vRNP transcription and replication. 10

1.6 Flow of mRNA to protein feedback loop facilitates vRNA production. 12

1.7 IAV cellular replication schematic. . . . . . . . . . . . . . . . . 14

1.8 IAV vRNP budding. . . . . . . . . . . . . . . . . . 15

1.9 Global overview of the dynamics at play during influenza A gene re-assortment. . . . . . . . . . . . . . . 17

2.1 Diagram of intracellular IAV infection. . . . . . . . . . . . . 31

3.1 Scheme of the influenza virus attachment. . . . . . . . . . . . 36

3.2 Comparison of fusion equilibrium for 1- and 2-mode attachment. . . 39

3.3 MM fits to bound and free virions vs total virions for multiple number of attachment sites. . . . . . . . . . . . . . . . . . . 42

3.4 Comparison of Heldt et al.'s MM and our MM fit to free and attached virions. . . . . . . . . . . . . . . . 43 
3.5 Comparison of virion entry of Heldt et al.'s and our MM . . . . . . 45

3.6 The percentage of virions fused out of total number of cell-associated virions. . . . . . . . . . . . . . . . . 46

3.7 Rate of endocytosis and nuclear import. . . . . . . . . . . . . . 47

3.8 Protein expression based on Heldt et al.'s MM. . . . . . . . . . . . 53

3.9 Comparing our MM and fitted parameters to experimental mRNA, vRNA and cRNA data. . . . . . . . . . . . . . . . . . 57

3.10 Single vs multiple cycle experiments. . . . . . . . . . . . . . 58

3.11 Comparing the two MMs to extracellular viral kinetics. . . . . . . . 60

3.12 Bound and free virions estimated with Heldt et al.'s MM. . . . . . . 61

3.13 Predicting viral titres for increasing input MOIs . . . . . . . . . 63

3.14 Effect of superinfection timing on production. . . . . . . . . . 64

3.15 Effect of early superinfection timing on production. . . . . . . . 65

3.16 Effect of superinfection dose on production. . . . . . . . . . 66

3.17 Antiviral mode of action. . . . . . . . . . . . . . . . 68

3.18 Effect of antiviral treatment at various efficacies. . . . . . . . . . . 70 


\section{Chapter 1}

\section{Introduction}

\subsection{Motivation}

The influenza A virus (IAV) causes a highly contagious infection of the upper respiratory tract. IAV has the potential to be responsible for large-scale pandemics, such as that by the H1N1 influenza A subtype in 1918, where 40 million people died worldwide [1]. The influenza A virus is a segmented negative sense RNA virus that belongs to the family of Orthomyxoviridae. It causes annual epidemics as it is easily transmissible. Worldwide it is estimated that there are 3 to 5 million cases of severe illness resulting in about 250000 to 500000 deaths per year. Those over the age of 65 , and children younger than 5 , but primarily younger than 2 years old are at highest risks of complications due to the respiratory infection. It is primarily spread by droplets from sneezing, and common symptoms include fever, nasal congestion, cough, runny nose, sneezing, sore throat, fatigue, and body aches $[2,3]$.

In Canada, for the 2015 - 2016 influenza season, there have been 5267 total influenza-associated hospitalizations and 251 deaths as reported by the Public Health Agency of Canada, as seen in Figure 1.1. Since influenza is easily transmissible, having infected patients in hospitals can put at risk vulnerable patients 
such as the young or immunocompromised hospitalized individuals [4].

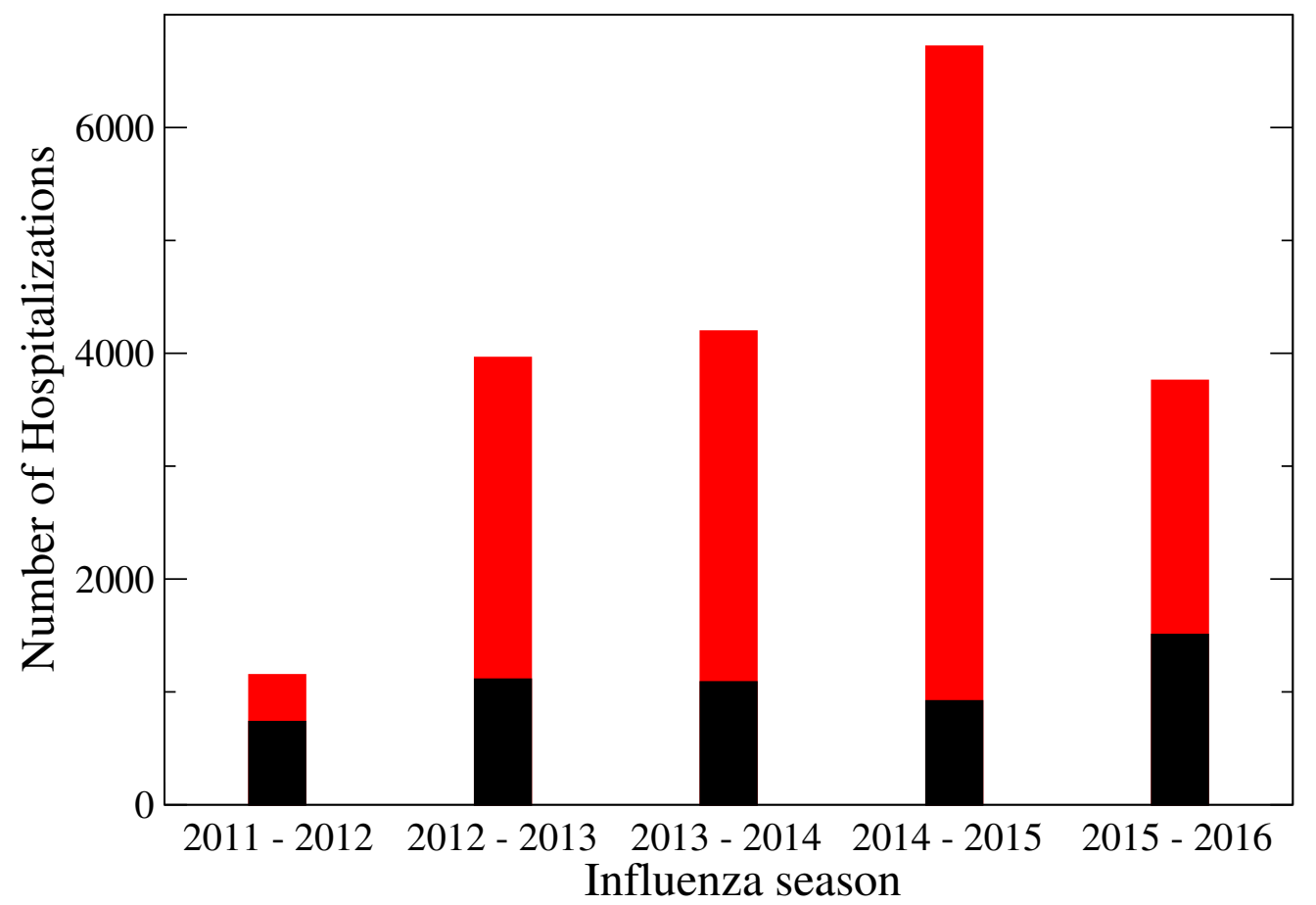

Figure 1.1: Reported annual Canadian hospitalizations. The number of paediatric hospitalizations (black bar, $\leq 19$ years of age) versus adult hospitalizations (red bar, 20+ years of age), deaths not reported in this figure. Reported values listed represent a portion of all influenza-associated hospitalizations of participating provinces and territories, which varies annually [4].

Resistance to common antivirals limits treatment strategies and places additional stress on the Canadian public health care system due to hospitalizations. FluWatch, a national surveillance network, was established in 2010 to facilitate sharing of knowledge between health professionals, as partly illustrated in Figure 1.1. Specifically, the aim of the network is to communicate data about prevalent circulating strains and any known sensitivities or resistances to antiviral treatments [4].

During virus infections there are complex interactions that exist between viral dynamics and the host cell defences. The use of MMs is beneficial in analysing infection. IAV has a high level of mutation, making it a moving target for an- 
tiviral treatment. As such, we must adopt faster ways of assessing the potential development and establishment of antiviral resistance in circulating strains. This is why MMs are so important: they can allow us to take given data and examine parameters not easily quantifiable through experimental means alone. With MMs, we want to accurately capture kinetics of IAV infection that incorporate current knowledge of influenza A with the long term goal to study the effectiveness of antivirals.

In the past, MMs have been used to determine the mode of action of antiviral compounds, to quantify their efficacy, but also to predict the outcome of combination therapy, where antivirals are combined as drug cocktails to reduce the chance of resistance emergence [5]. MMs have been used to study and describe infection with the human immunodeficiency virus (HIV-1), and have contributed immensely to the development of all aspects of understanding infectious diseases; from basic biology and kinetics of infection, to improved treatment strategies. In 1996, Perelson et al. modelled the infection of five HIV-1 patients before and after treatment with a HIV-1 protease inhibitor. Their findings estimated that HIV1 production was greater than previously thought, and that its generation time (time from leaving one cell to infecting another) was also longer than expected. These results illustrate the importance of treating early, as increased production leads to viral diversity, and can lead to reduced efficacy of therapy [6]. MMs can be adjusted quickly to examine fast evolving strains, and provide prediction on infection kinetics.

As a starting point, we will consider the simple delayed virus production MM presented in Figure 1.2 which has been used extensively before to model in vitro viral infections with influenza $\mathrm{A}$, hepatitis $\mathrm{C}$ virus, and HIV. The invading virus $(V)$ infects susceptible epithelial target cells $(T)$ at a rate of infection $\beta$. Those cells then go through an eclipse phase $(E)$, for a given time denoted as $\tau_{E}$, during which viral replication occurs but no progeny virus is being released. After the eclipse phase, once new virus is ready to be released, cells enter the infectious $(I)$ phase. Infectious cells are presumed to be able to produce new virus, $V$ at a rate denoted as $p$. The initial infecting virus and newly produced virus, are subject to a viral clearance rate $(c)$ due to loss of infectivity. In contrast to in vivo 


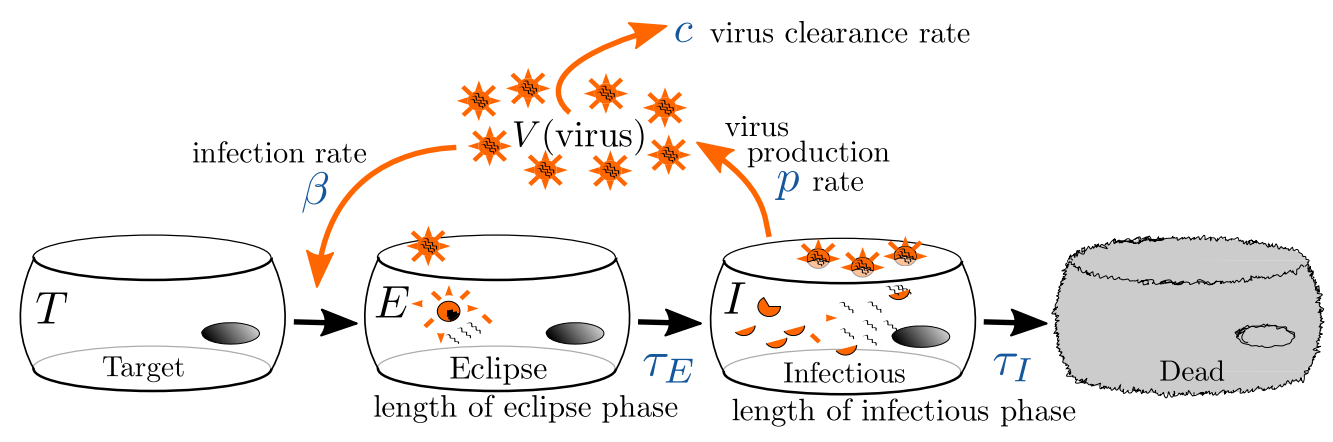

Figure 1.2: Mathematical model for influenza viral infection in vitro. An illustration of the standard MM of influenza A virus infection from infection to release [Courtesy of Ben Holder] [7].

experiments which consider clearance by the host immune system, in vitro loss of infectivity is primarily viral-dependant. The factors for reduced infectivity range from degradation of the physical virion to degradation through cellular pathways. The time after the cell becomes infectious until cell death $(D)$ occurs, is denoted as $\tau_{I}[5,8]$. The MM in Figure 1.2 is an illustration of the explicit MM represented by the following equations:.

$$
\begin{aligned}
\frac{\mathrm{d} T}{\mathrm{~d} t} & =-\beta T V \\
\frac{\mathrm{d} E}{\mathrm{~d} t} & =\beta T V-\frac{E}{\tau_{E}} \\
\frac{\mathrm{d} I}{\mathrm{~d} t} & =\frac{E}{\tau_{E}}-\frac{I}{\tau_{I}} \\
\frac{\mathrm{d} V}{\mathrm{~d} t} & =p I-c V
\end{aligned}
$$

The MM does not explicitly consider intracellular replication as here, only the cell-to-cell component is considered. In this multicellular MM the IAV infection is tracked through multiple cells. The initial conditions of the experiment, whether infecting only a portion or all cells in a culture, plays a large role in how the infection will progress. These MMs neglect the complexity of intracellular replication e.g., production of proteins and progeny virus segments, trafficking of viral pro- 
teins, and assembly of the virus and the virus production rate are often simplified to the length of the eclipse phase.

For IAV replication there currently exists other MMs. Later I discuss the work of Sidorenko et al. and Heldt et al., published in 2004 and 2012 respectively, which both show good replication of data, but still have points of possible improvement, i.e. over-complication or over-simplification of some key concepts $[9,10]$.

\subsection{Goal}

MMs have been shown to serve as valuable tools with which to study IAV. The immediate goal addressed by the present project is the development of a robust and concise MM of intracellular IAV replication within a single cell which agrees with the most current knowledge of biological events during infection with IAV.

Antivirals are prescribed to patients during an IAV infection and have the ability to reduce the severity of symptoms. They have the greatest effect the earlier they are administered after onset of infection. However, IAV is constantly mutating and recombining, and can develop or acquire mutations making it resistant to antivirals, making treatment less effective. The MM must be detailed enough so that each process which can be the target of antiviral therapy is explicitly represented, yet it must remain simple enough that it can be integrated into millions or more cells to simulate the infection of a whole cell culture. The existing intracellular MM by Heldt et al. is too large and complex to be realistically used to simulate and study infection of a cell culture, representing or tacking upwards of $10^{6}$ cells per experiment.

Secondary goals of this thesis were to begin to answer critical questions regarding intracellular IAV replication which, are difficult or impossible to address or probe experimentally. We seek to answer questions related to cells infected with multiple virions i.e. superinfection. We examine whether a superinfected cell will be able to produce progeny virions faster, or if will it produce more virions in total but in the same time as if infected by a single virus. We evaluate the effect of 
multiple secondary inoculum to effect infection kinetics, by varying the timing and dose of the secondary inocula. This is primarily important as virus recombination during co-infection, and the production of defective interfering and non-interfering particles as a results of errors and/or assortments, is dependant upon dose and timing.

This MM will consider a single infection cycle and neglect virus spreading through virions to other cell, as this MM considers only intracellular infection within a single infected cell. Experimental infection data acquired from literature are preformed under varying conditions, and thus will likely have variations not solely due to IAV. It is important to note that the although in vitro studies have the ability to provide insight of the response of a whole organism, any conclusions made will require extensive testing to determine real world relevance.

Since the ultimate goal is to develop a better MM to describe the process of intracellular IAV replication within a single cell, construction of MM required an understanding of the steps involved in the viral replication cycle of influenza A virus. Specifically, it required detailed knowledge of (1) attachment, (2) viral entry, (3) synthesis of viral RNA, (4) synthesis of viral proteins, (5) packaging of RNA and assembly of virus, and (6) virus budding and release. This was accomplished through diligent literature review and comparison of in vitro data for infection in cells. We refined the accuracy of parameters through analysis and validation of results. Additionally, we interpreted our results by comparing and contrasting them to known values. This thesis will combine the following key aspects: a literature survey, a theoretical assessment, a coding/modelling project, and extensive testing with IAV experimental results.

\subsection{Influenza A virus structure and replication process}

The influenza virion is composed of 8 single stranded negative sense ribonucleic acid (RNA) segments, coated in viral proteins, and enclosed in a lipid bilayer, and 
encode for up to 11 proteins. The 8 genome segments, listed here from longest to shortest, are: polymerase B2 (PB2), polymerase B1 (PB1), polymerase A (PA), hemagglutinin (HA), nucleoprotein (NP), neuraminidase (NA), matrix (M) which encodes for matrix protein 2 (M2), and lastly non-structural (NS) segment. The last segment encodes for two proteins; the non-structural protein 1 (NS1) and the nuclear export protein (NEP), formally referred to as the non-structural protein 2 or NS2 protein. Imbedded in the lipid bilayer, which coats the viral nucleoclapsid, are the two glycoproteins: hemagglutinin, and neuraminidase, and the transmembrane proton channel, the matrix protein 2, as illustrated in Figure 1.3 [11].

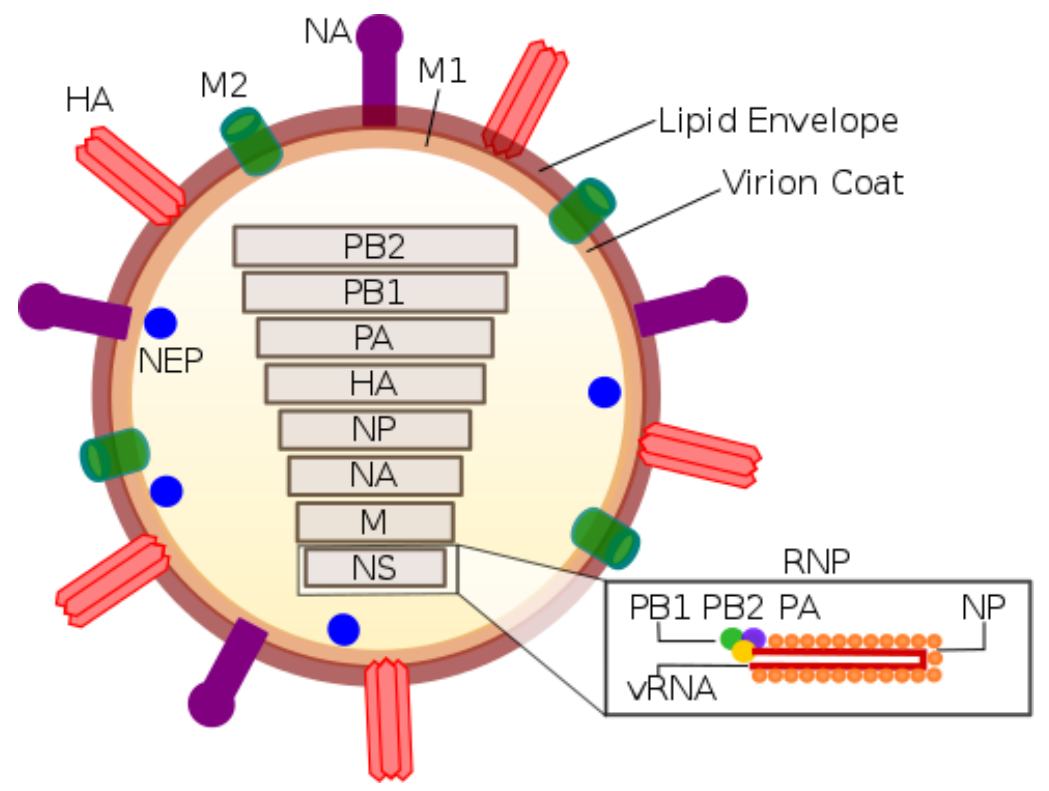

Figure 1.3: Influenza A virus. The proteins encoded by PA, PB2, and PB1 are internal proteins responsible for viral replication, for mitochondrial targeting and apoptosis. HA is a surface glycoprotein responsible for the attachment of the virus to host cells, while NA is responsible for the release from host cells through cleavage of cell surface receptors. NP is required for RNA coating and stabilization and in particular, PB1 is responsible for RNA transcription. Matrix proteins M1 and M2 aid in membrane stability, and viral uncoating, respectively, while non-structural proteins, NS1, is an internal protein. NS2 functions to regulate the virus life cycle, specifically mRNA transcription and localization of viral ribonucleic proteins. Each segment is coated with NP proteins and a vRNPs $[11,12]$ 
Recent advances in high-resolution live microscopy have enabled the development of new approaches towards a better understanding of the cellular machinery in IAV infections. The segments present inside the virus are negative sense RNA and cannot be immediately translated into proteins. First, the vRNA is transcribed into a complementary positive sense mRNA strand by RNA-dependent RNA viral polymerase. Later, the primary vRNA will also serve as a template for complementary RNA (cRNA) and the creation of progeny viral RNA (pvRNA) $[11,13]$.

Each genome segment consists of a negative RNA segment coated with NP proteins topped by a viral polymerase complex referred to as viral riobonucleoproteins (vRNP), which adopts a corkscrew shape, as seen in Figure 1.4. The PB1, PB2, and PA are the subunits which make up the viral RNA polymerase. Each part of the RNA polymerase complex must be fully functional for replication to proceed correctly. The polymerase present in influenza can both transcribe positive sense messenger RNA (mRNA), and replicate positive cRNA, which results in pvRNA, and finally as progeny viral RNP (pvRNP), as explained in Figure 1.5 [11].

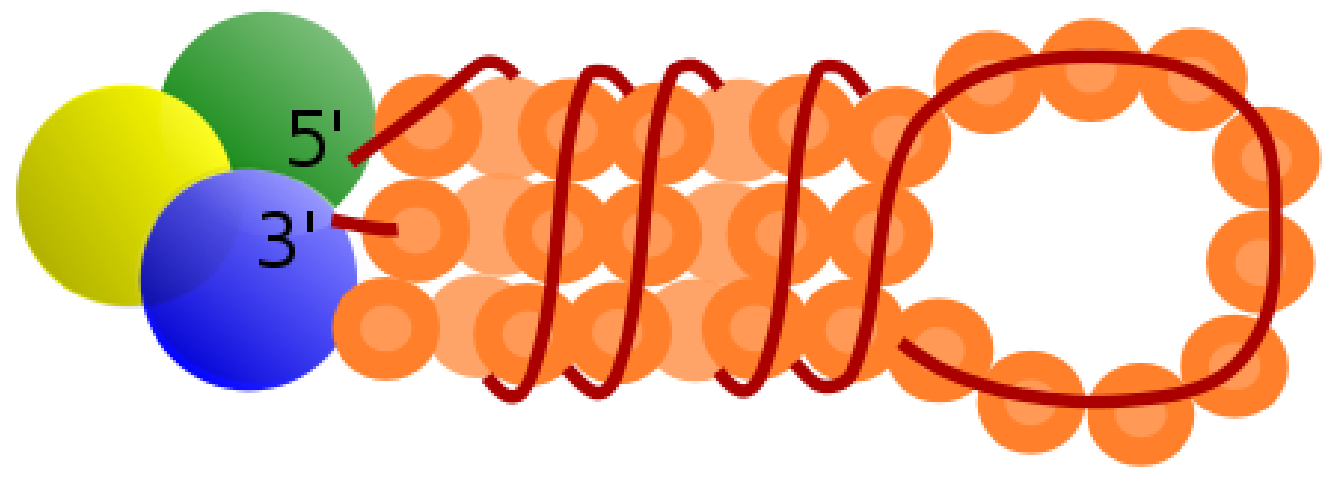

Figure 1.4: vRNP structure. Inside the virus, the genome segments are packed in the form of vRNPs. These consist of negative sense RNA (red line), where for every 24 nucleotides, there is one NP protein (orange spheres) to keep the RNA from interacting non-specifically and degrading. The viral polymerase complex, referred to as a viral ribonucleoprotein, vRNP, is made of PB1, PB2, and PA (yellow, green and blue spheres) [Adapted from Infante et al. 2011] [12-14].

The cork screw viral ribonucleoproteins (vRNP) structure, shown in Figure 1.4, is illustrated with the polymerase and the negative sense vRNA [13]. In order for 
the polymerase to start transcription of vRNA to mRNA it requires a primer $(\approx$ 15-20 amino acids in length) which it steals from the host cell via a process called cap snatching. The cap helps viral mRNA avoid degradation. The polymerase attaches the cap to the start of the positive sense mRNA segment and begins transcription, until it gets tangled at the end loop structure and stutters on the U codon of the negative sense. Consequently the polymerase reads the same codon over and over which produces a poly A tail, i.e. a long segment of A codons get added at the 5' end of the mRNA segment. As such, the positive mRNA produced is shorter than the original negative sense vRNA. The vRNA can coil back up and the process can repeat many times to produce many positive mRNA that will go on to produce proteins $[11,13]$. The rate limiting step in protein production is the rate of ribosome attachment to the mRNA. 


\section{Transcription}
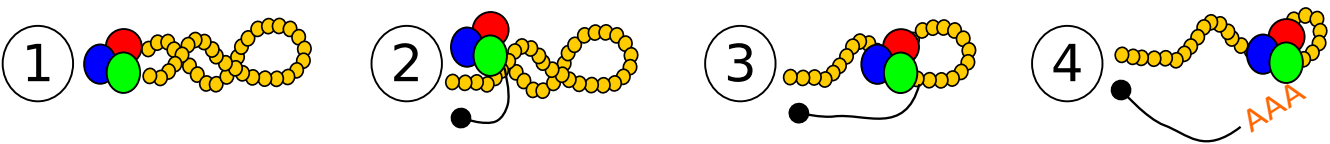

Translation

(1)

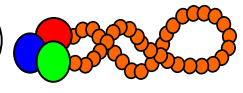

(4)

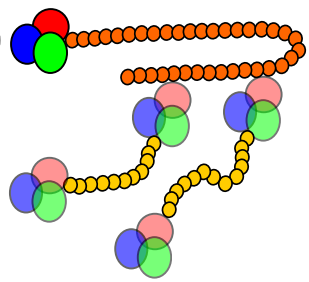

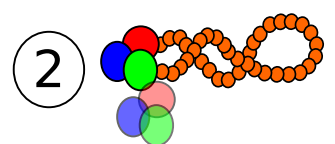
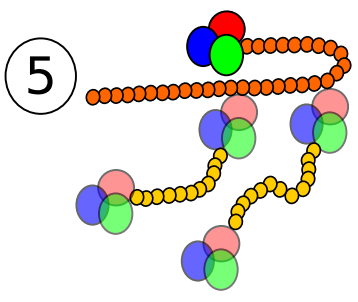

(3)

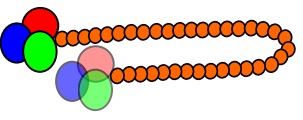

(6)

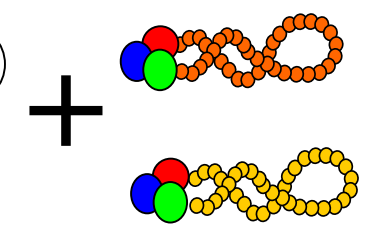

viral polymerase

- mRNA primer

- mRNA

progeny polymerase - 0000 VRNA template (-) $\infty 00000$
AAA amino acids cRNA template $(+)$

Figure 1.5: Differences in the mechanism of vRNP transcription and replication. The switch between transcription and replication is dependant upon the stabilization of cRNA (red) which is needed in order to produce pvRNPs (yellow). A notable difference is also that transcription requires a 5' cap and cannot replicate itself completely though, while for cRNA a complete copy is made, although the details regarding this step are not yet fully understood [Adapted from Jorba et al. 2009] [13,15]. 
When vRNA is used to produce pvRNPs, the process differs from transcription of mRNA in the fact that cap snatching does not occur, and that newly synthesized polymerase, rather than the segments own polymerase, are capable of reading though to the end of the template. Although the whole process is not fully understood, it is known that a progeny polymerase attaches to the 3' end and detangles the cRNA coil. The polymerase then begins to read along the cRNA, producing new pvRNA, and a second progeny polymerase attaches to the 5' end of the pvRNA. Several new polymerases can attach to the vRNA at one time [13].

There exist two theories currently on how the virus controls switching from transcription of mRNA to translation of cRNA. In the classical theory, it is believed that the virus produces only mRNA for a certain time, and then switches to producing cRNAs. More recently, it has been shown that cRNA can be detected at early time points, not consistent with the switching hypothesis. Using primer extension analysis, Vreede et al. showed when transfected with viral RNA prior to infection, the cell expresses detectable levels of cRNA at the same time as mRNA [16]. These findings suggest that cRNA is possibly produced as early as, and potentially even competes with, transcription of mRNA. The low, and previously undetectable levels of cRNA compared to mRNA, would result from the fact that cRNA lacks certain proteins, only imported into the nucleus at later time points. Thus cRNA would degrade rapidly early in the infection since it requires these proteins for stabilization [13]. This hypothesis is illustrated in Figure 1.6. 


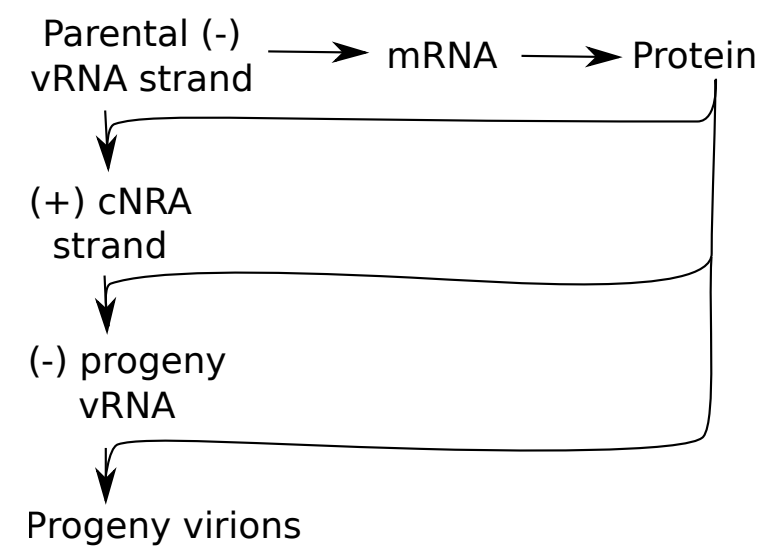

Figure 1.6: Flow of mRNA to protein feedback loop facilitates vRNA production. The switch between transcription/translation is mediated by newly synthesized proteins entering the nucleus to stabilize cRNA. The feedback loop of viral proteins into the nucleus allows for vRNA production [2].

The steps involved in IAV replication, illustrated in Figure 1.7, are as follows:

Attachment : IAV infects a host through a binding interaction of the viral glycoprotein hemagglutinin with sialic acid on the surface of host cells. The virus is then pinocytosed into an endosome via clathrin receptor mediated endocytosis [11]

Viral entry: After the virus is endocytosed the endosome is acidified. The acidic environment is caused by (1) Hemagglutinin fusing the endocytotic vesicle and the virus coat creating a pore and (2) the influx of protons pumped into the viral envelope via the M2 ion channel. The acidification event results in the fusing of the endosome plasma membrane to the viral envelope which results in the uncoating and release of the eight viral RNP segments and core proteins to the cytoplasm. Those with defective M2 ion channels are degraded by the lysosome. Upon their release they are transported to the nucleus due to the exposure of the vRNP nuclear localization signals [11,17].

Synthesis of Viral RNA for Viral Proteins: Transcription After the viral RNA and viral replicase enter the nucleus the viral genome is converted by a viral replicase into a positive sense mRNA. The positive sense mRNA strand takes a mRNA 
cap from cell to add to the newly synthesized mRNA - a process known as cap snatching. The capped viral mRNA strands now mimic host mRNA and is trafficked through the endoplasmic reticulum where it is translated into proteins by host machinery. Transcription of DNA to mRNA results in immature mRNA. The introns are spliced out and the exons are used, resulting in mature mRNA (not shown). Translation of mRNA requires association of the small and large ribosomal subunits to the 5 prime end of the RNA. The Ribosome scans the RNA till a start codon is found. It translates it in 3 amino acids at a time into protein by recruitment of tRNA till it reaches a stop codon (UAG) tRNA's enter at the A site, move to the $\mathrm{P}$ site where the incoming peptide works a bond with the growing peptide chain $[13,18]$. Post-translational processing exposes a short leader peptide on the viral proteins, which depending upon the signal sequence the viral protein, can be directed to the cell surface or back to the nucleus. Those coding for membrane proteins, neuraminidase, hemagglutinin and M1, are trafficked to the host cell membrane where they accumulate. Several of the newly synthesized viral proteins, M1, are trafficked back to the nucleus where they accumulate (increase in concentration) and cause the viral protein replication process to end and allow for the creation of viral progeny RNA $[11,13]$.

Synthesis of Viral RNA for Progeny: Translation Inside the nucleus the initial vRNA is a template for a positive sense complementary RNA (cRNA) which serves as a template for progeny vRNA. Newly synthesized proteins such as NP, M1 and NS1 require trafficking into the nucleus, where they bind to newly synthesized negative strand vRNA. The proteins NP, M1 and NS1 bind to newly synthesized negative strand viral RNA, creating viral progeny nucleocapsids that induce export to the cytoplasm via NEP where they converge with the membrane proteins $[11,14]$. 


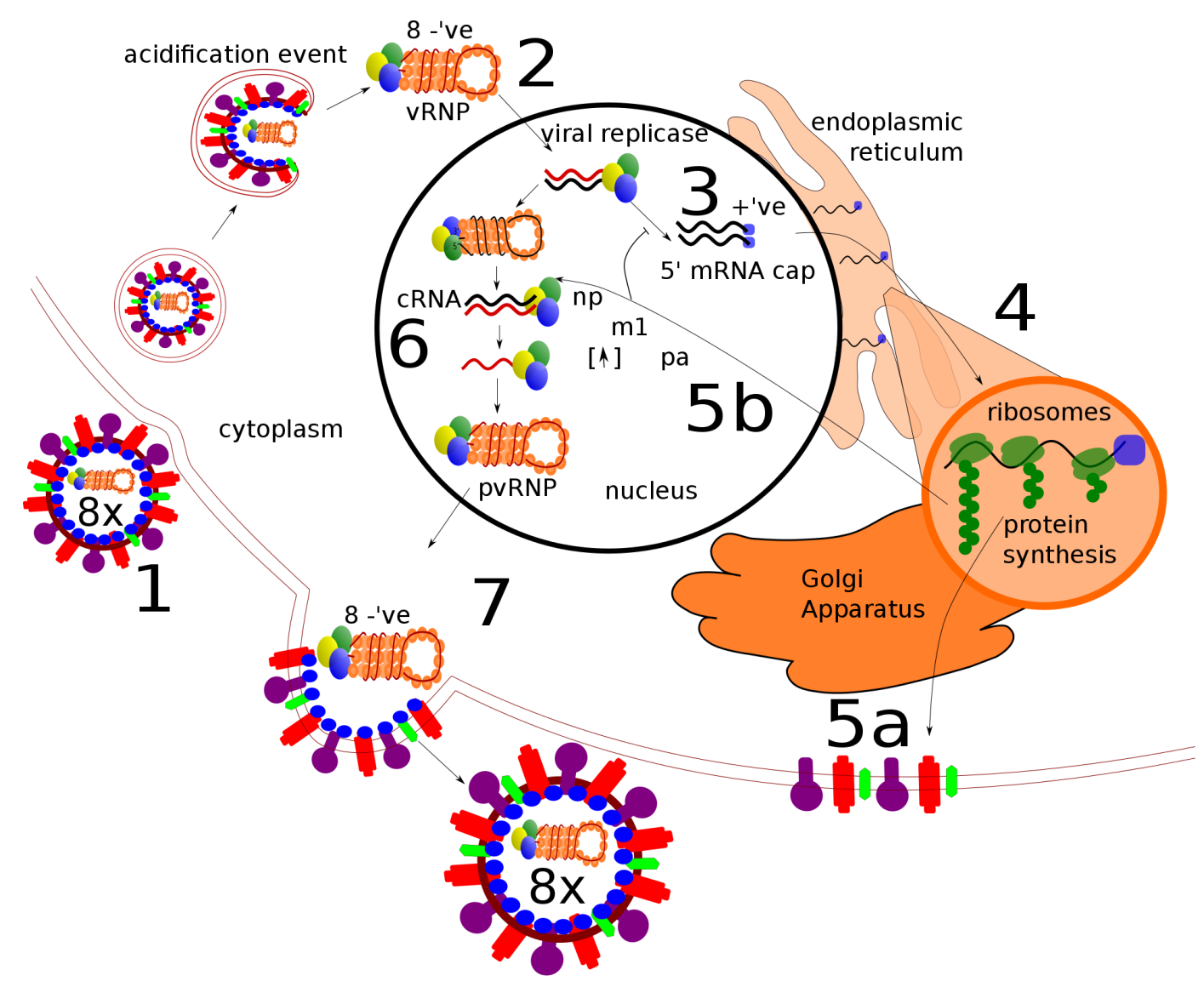

Figure 1.7: IAV cellular replication schematic. Simplified order of events: 1) Free virus attached to receptors on the cell surface. The virus, along with the cell membrane, is endocytosed via receptor-mediated endocytosis which then releases 8 negative vRNPs into the cytoplasm. 2) The 8 negative sense vRNPs strands are imported into the nucleus along with viral replicase (RNA-dependent RNA polymerase). 3) Negative strand is transcribed into positive sense strand and takes a mRNA cap from cell to add to the newly synthesized positive sense strand - a process known as cap snatching. 4) The viral mRNA strands now mimic host mRNA and are trafficked through the endoplasmic reticulum where they are translated into viral proteins by host machinery. 5a) Membrane viral proteins, HA, NA, and M2 are trafficked to the cell surface where they are integrated to the cell membrane. 5b) Viral proteins, such as PB1, PB2, PA, and NP, are trafficked into the nucleus. Viral protein accumulation in the nucleus is a requirement for viral translation to occur as these proteins stabilize progeny cRNA and vRNA. 6) NP, M1 and NS1 bind to newly synthesized negative strand viral RNA, creating viral progeny nucleocapsids. Together NP, M1 and NS1 induce nuclear export to the cytoplasm via NEP. 7) The progeny nucleocapsids converge where HA, NA and M2 have accumulated on the cell membrane. Budding occurs followed by NA cutting cellular receptors allowing the bud to be released from the host cell $[11,14,17,19]$. 
Packaging of RNA and assembly of virus: The progeny nucleocapsids acquire an envelope and converge where HA, NA and M2 have accumulated on the cell membrane, shown simplistically in Figure 1.8. Unique vRNPs are selectively incorporated into progeny viral particles due to their segment-specific non-encoding regions, protein coding regions, and promoter region, conserved between the $\mathrm{vR}$ NAs. When packaged, the vRNPs acquire a $7+1$ configuration prior to budding reliably, as explained in further detail in references [19-21]. In addition to the production of standard virus (fully functional), IAV also produces defective virions which, may be infectious or uninfectious. Some infectious defective virions are unable to complete replication, i.e. production of progeny virions in a new cell would be impaired. In this scenario, defective virions have incomplete or mutated vRNP segments resulting in missing or misfolded proteins required for replication. If two genetically different viruses infect the same cell at the same time both replicate simultaneously causing extensive mixing $[11,19,22]$.

Virus Budding and Release: Budding begins and release occurs due to neuraminidase enzymatically cleaving sialic acid from viral glycoproteins, thereby allowing it to bud.

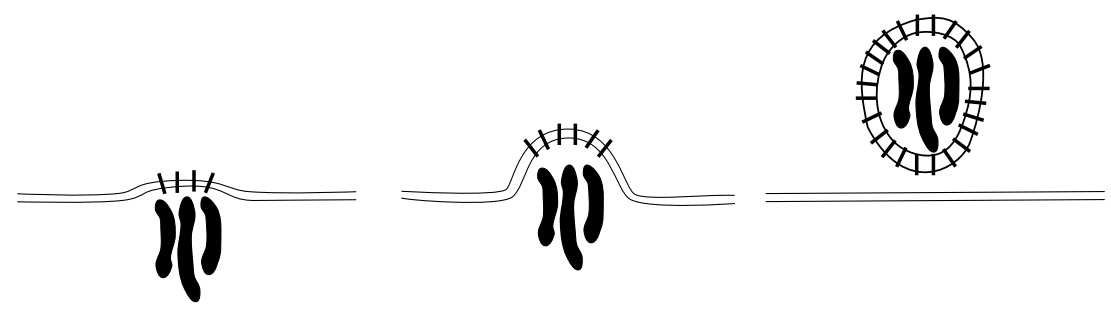

Figure 1.8: IAV vRNP budding. After nuclear export, the 8 segments of vRNP accumulate at the plasma membrane where the viral transmembrane proteins have been integrated. The vRNPs accumulate in a $7+1$ conformation as the virus begins to bud, as illustrated by Zheng et al. 2013 [19] [Adapted from Zheng et al. 2013].

IAV has a history of mutations of its surface glycoproteins leading to subtypes, the first one isolated as H1N1, and the second in 1958 with H2N2, named so due to the subtype of hemagglutinin and neuraminidase present on the surface. Currently there are 18 HA subtypes and 11 NA subtypes known for IAV [1,23]. Each subtype 
has preferences for different terminal glycans on the cell surface. These specificities change from year to year due to genetic drift, resulting in different strains within a subtype [1].

Cell type can affect infection kinetics as different cell lines express surface glycans at different ratios. The activation of CD69 was greater for Vero cell derived glycovariants than MDCK cells as determined by flow cytometry. The variants produced in Vero cells resulted in fewer glycan structures with lower molecular masses, dominated by terminal $\beta$-galactose, while the MDCK variants had terminal $\alpha$ and $\beta$-galactose, no high-mannose glycan structures and tri- and tetraantennary structures. Cells can, however, be transfected to express levels of $\alpha-2,6$ indicative of human epithelial airways [24]. Testing in animals, such as mice and ferrets, also presents certain shortcomings due to species differences (such as cell glycosylation), but they can provide a great tool in studying the transmission capabilities of a IAV strains.

Animal reservoirs, such as pigs or birds, are distinct and often humans lack innate immunity towards them, as illustrated in Figure 1.9. These mixing events are of major concern, especially in pigs, as they provide a means of combining avian with human IAV [3]. There are regions of HA known to facilitate the species receptor binding specificity switch and increase transmissivity. For example, Gln 266 is responsible for forming hydrogen bonds with the receptor, sialic acid. A Leu ${ }^{266}$ mutation, which does not hydrogen bond with sialic acid, changes the Gal-2 binding pocket of HA, resulting in increased affinity to human $\alpha-2,6$ sialic acid [26].

After IAV infection antivirals can be used to treat patients. When taken within the first 2 days of infection, antivirals can lessen the symptoms associated with the infection, and for those with previously compromised health, can be the difference between a mild illness or a hospitalization. Antivirals require extensive testing to effectively target viral processes and minimizing toxic side effects as there is an intricate regulation of viral and host proteins during IAV infection. Examining the effect of antivirals on the kinetics of infection can provide us with unique perspective on the mechanism behind the infection and allow us to evaluate if our MM can correctly reproduce viral infection kinetics [23]. 


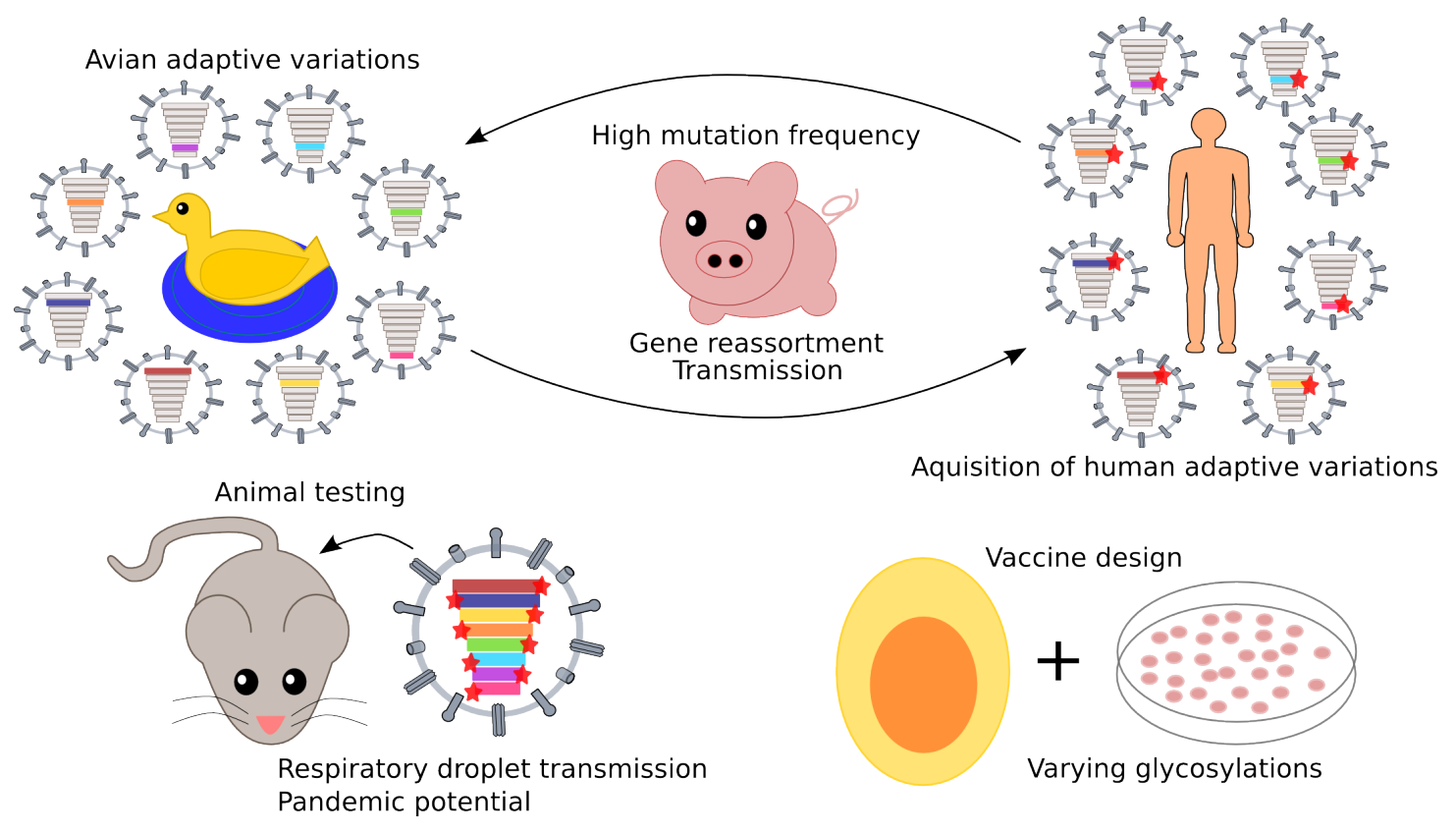

Figure 1.9: Global overview of the dynamics at play during influenza A gene re-assortment. Avian vs human preference is mediated by HA binding to SA, which can be altered by levels of HA glycosylation. Humans are naïve in the glycan required by avian strains to infect, but as influenza evolves it increases the possibility of obtaining certain mutations that can change its species preference, mode of transmission and virulence. This illustrates briefly the species level of complexity, including testing in animals and vaccine development, and some key features of a pandemic potential strain [1,25].

Adamantanes, such as amantadine and rimatadine are uncoating inhibitors which block the M2 protein channels required for release of viral RNA into the cytoplasm. The acidification in the endosome due to proton pumps causes HA to undergo a conformational change which exposes it to host cell proteases. HA proteolytic cleavage is required by host cell proteases for release of the eight viral segments: if HA is not cleaved, infection will not progress. Approximately $51 \%$ of endocytosed virions get degraded before reaching the nucleus and starting replication $[11,27]$. Proton activity is mediated by a single transmembrane domain. Mutations in the transmembrane region of the M2 protein can lead to resistance to amantadines. Known sites of mutations are Leu26, Val27, Ala30, Ser31 and Gly34 [28, 29]. 
Amantadine causes neurological side effects in humans due to its dopaminergic and adrenergic activity, and to its activity as an anticholinergic. Treatment of IAV with fusion inhibitors can cause cardiac arrest, acute respiratory failure, muscle spasms, personality changes and seizures. However, such adverse affects are uncommon and are often present only in the elderly or those with pre-existing medical condition [30]. In recent years, adamantanes have ceased to be prescribed due to conferred resistance of the prominent yearly strain. During the 2003-2004 season, IAV resistance to adamantanes increased 30-fold.

Often, but not always, the mutation which allowed the IAV strain to be resistant to an antiviral is paired to negatively affected fitness. This resistance can be conferred from one strain to another, especially during re-assortment, and can result in a strain possessing immunity and remaining fit. Monto et al. showed that on tissue culture grown in the presence of amantadine, a single round of passaging of IAV infected cells was sufficient to select for amantadine resistance. These kinds of studies provide some insight into how the virus will evolve, especially when grown in the presence of an inhibitor such as amantadine [31]. Some mutations are beneficial to the virus as they might confer resistance, lead to an increased virulence result in a mutation there was loss in fitness.

The most prominent neuraminidase inhibitors that target the release of progeny virions currently are zanamivir (Relenza ${ }^{\mathrm{TM}}$ ) an aerosol, and oseltamivir (Tamiflu ${ }^{\mathrm{TM}}$ ) an oral pro-drug. Neuraminidase normally cleaves the terminal sialic acid from transmembrane glycoproteins on both host and viral envelopes. The inhibitors are sialic acid analogues that block the active site of neuraminidase resulting in an aggregation of uncleaved sialic acids which prevent the virus from budding off $[23,32]$. Through modification and substitution of the sialic acid backbone, small molecules functioning as inhibitors are produced which are capable of fitting into the binding pocket of neuraminidase. Zanamivir, is one such example, which posses a substitution of a guanidine group for the 4-hydroxyl group in sialic acid. This resulted in increased binding affinity due to guanidine group interactions between carbonyls Asp151 and Trp178 in the main chain, acidic residues Asp151 and Glu227 in the side chains, and hydrophobic stacking with Glu119. Zanamivir has a higher binding affinity than oseltamivir. For oseltamivir, the glycerol is substituted by 
hydrophobic l-ethylpropoxy group and the guanidine group with an amine. These substitutions resulted in reduced polarity and increased lipophilicity. Zanamivir and sialic acid both have O-H...O type interactions with Glu276, and hydrogen bond to the glycerol with Glu276, while oseltamivir's ethylpropoxy group forms hydrophobic stacking with Glu276. In 2009, there were reported resistances to oseltamivir, which luckily had a limited widespread impact. These values vary from year to year depending upon the characteristics of the dominant IAV circulating strain in that season [23]. Oseltamivir resistance has developed through H274Y and R292K substitution, the latter causes a low level resistance to zanamivir also [29].

A common broad spectrum antiviral is nucleoside inhibitor ribavirin (Virazole ${ }^{\mathrm{TM}}$ ) which, acts as a nucleoside analogue for both RNA and DNA synthesis. As a pro-drug, ribavirin requires activation through cellular metabolism, specifically phosphorylation by cellular nucleoside and nucleotide kinases. After ribavirin is metabolized in the body, its structure resembles purine nucleotides: adenosine or guanosine. Activated ribavirin get competitively misincorporated into vRNA and mRNA by viral-dependent RNA polymerases. Depending of the rotation of the carboxamide moiety of this guanosine analogue, it can pair with both cytosine and uracil equally. For mRNA synthesis the PB2 subunit (residues 318-482) bind the 5'cap of host pre-mRNA, cleaves the phosphodiester bond 10-13 nucleotides downstream of the cap and initiates transcription of viral mRNAs. Misincorporations which lead to amino acid changes can result in misfolded and non-functional proteins $[28,29]$.

A new antiviral, favipiravir (T-705), approved in Japan for treatment of IAV, has shown to be effective against IAV strains resistant to current antivirals. Favipiravir has been shown effective on abroad range of other RNA viruses. Activated favipiravir, favipiravir-RTP, is mistakenly recognized by viral RNA-dependant RNA polymerase as a purine nucleotide and halts replication. Treatment of mice infected with IAV H3N2 with favipiravir and favipiravir-oseltamivir treatment therapies resulted in increased survival in mice treated with the combination therapy $[33,34]$. 
Our MM must reproduce IAV replication data, namely the production of virions and RNA. A constraint on our MM is that it be sufficiently detailed to capture the modes of action of all the different antivirals outlined in the scope of this thesis. 


\section{Chapter 2}

\section{Methods}

\section{$2.1 \quad$ Experimental data}

It is important to note that different IAV strains used in different studies and can have an effect on viral load and other parameters. In some circumstances, we only compare kinetic timing and not specific values. When studying IAV infection of cells, the cell line used can have an impact on the infection kinetics. The studies we examine were all done with Madin-Darby Canine Kidney (MDCK) epithelial cells, a cell line commonly used for modelling human epithelial airway conditions

The following data from literature was used in the present work for parameter extraction or comparison of kinetics:

Nunes-Corria et al. 1999 [35] This experiment was used for its data for attachment and detachment rates of IAV. MDCK cells were pre-incubated for 30 minutes with antimycin A to inhibit endocytosis, thus only measuring attachment/detachment rates. Various inocula of IAV A/PR8/34 were added to MDCK cells in culture. At 20 minutes post infection, cells were centrifuged for 8 minutes at $180 \mathrm{~g}$ at $4^{\circ} \mathrm{C}$. Fluorescence of the supernatant (free virions) and of the pellet (attached virions) following detergent lysis with Triton X-100 were measured at $37^{\circ} \mathrm{C}$. 
Stegmann et al. 1995 [27] From this experiment, it was seen that 51\% of virions are fusion competent, and our MM should reflect this. In brief, R-18 labelled influenza virus (strain NIB26) was added to MDCK cells $0^{\circ} \mathrm{C}$ for $2 \mathrm{~h}$, to allow for virus adsorption, and washed so that unattached R-18 was removed. Warm buffer $\left(37^{\circ} \mathrm{C}\right)$ was added, then viewed under florescence microscopy with continuous stirring. The percentage of fused virions out of total cell-associated virions was measured.

Kawakami et al. 2011 [36] These experiments resulted in the acquisition of RNA data up to 12 hours post infection at 100-100,000-fold higher than untagged RNAs. MDCK cells were infected with influenza virus A/WSN/33 (H1N1) strain at an MOI of 10 in triplicate. Average numbers of RNA copies in an infected cell were determined by strand-specific real-time RT-PCR.

Holder et al. 2011 [37] MDCK cells were modified to increase sensitivity to neuraminidase inhibitors and to express $\alpha-2,6$ sialic acid receptors at levels indicative of human epithelial tissue. These ST6Gal I-MDCK cells were grown to confluence and infected with A/Brisbane/59/2007 (H1N1) at an MOI of 1 and adsorbed for $1 \mathrm{~h}$ at $37^{\circ} \mathrm{C}$. Cells were washed three times then supernatant replaced, thereafter plaque forming units per $\mathrm{mL}$ were counted. Two replicates of two independent experiments were preformed.

Paradis et al. 2015 [38] MDCK cells were modified to express $\alpha-2,6$ sialic acid receptors (MDCK $\alpha 2,6)$ consistent with human epithelial airways. MDCK $\alpha 2,6$ were infected in triplicate with 2009 pandemic H1N1pdm09 WT-I223V strain A/Québec/144147/09 at an MOI of 4. Virus was allowed to adsorb for $1 \mathrm{~h}$ at $37^{\circ} \mathrm{C}$, washed three times, and medium replaced. Supernatant was collected up to 18 hours post infection. 


\subsection{Mathematical modelling}

Data used to test our MM were taken from literature sources dating from 1995 to 2015. Data was digitized using Engauge Digitizer 5.2., a digitizing software which converts an image file of a graph into numbers which were saved as .dat files. The data was used to calibrate and validate our MM, coded in Octave [39]. The experimental conditions from literature previously described are separated into those which affected the MM directly, or were used to evaluate MM predictions for the extracellular viral titers.

Parameters were determined using the Nelder-Mead minimization iterative algorithm (nelder mead min) in Octave. The algorithm iteratively finds the minimum of a non-linear function, then restarts until no improvement is found. The determination of a best fit is through the computation and minimization of sum of squared residuals (SSR), which are deviations from the experimental data and the calculated MM at matching time points [39].

In Heldt et al.'s MM, free virions $V^{\mathrm{Ex}}$ attach to free binding sites of low $\mathrm{B}_{\mathrm{lo}}^{\text {tot }}$ at a rate of $\mathrm{k}_{\mathrm{low}}^{\text {Att }}$ and high affinity $\mathrm{B}_{\mathrm{lo}}^{\text {tot }}$ at a rate of $\mathrm{k}_{\mathrm{hi}}^{\mathrm{Att}}$. For $\mathrm{V}_{\mathrm{lo}}^{\mathrm{Att}}$ and $V_{\mathrm{hi}}^{\mathrm{Att}}$ once attached, free virions can either dissociate from the cell or enter into the cell through endocytosis. They dissociate with the rates $\frac{\mathrm{k}_{\mathrm{lo}}^{\mathrm{Att}}}{\mathrm{k}_{\mathrm{lo}}^{\mathrm{Eq}}}$ and $\frac{\mathrm{k}_{\mathrm{hi}}^{\mathrm{At}}}{k_{h i}^{E q}}$, with $k_{\mathrm{hi}}^{\mathrm{Eq}}$ and $k_{\mathrm{lo}}^{\mathrm{Eq}}$ being the equilibrium constant. For entry, the virions enter the cell with the endocytosis rate, $\mathrm{k}^{\mathrm{En}}$, and is assumed to be the same for both binding sites.

After endocytosis a fraction of the virions which are fusion competent, $\mathrm{F}_{\text {Fus }}$, either fuse with the endosomes releasing 8 segments into the cytoplasm, $\mathrm{Vp}^{\text {cyt }}$, at a rate of $\mathrm{k}^{\text {Fus }}$, or degrade at a rate of $\left(1-\mathrm{F}_{\text {Fus }} / \mathrm{F}_{\text {Fus }}\right) \mathrm{k}^{\text {Fus }}$. Cytoplasmic vRNP

segments are then imported into the nucleus, $\mathrm{Vp}^{\text {nuc }}$ at a rate of $\mathrm{k}^{\mathrm{Imp}}$, due to dissociation of M1 protein. 


$$
\begin{aligned}
& \overbrace{\frac{\mathrm{d} V^{E x}}{\mathrm{~d} t}}^{\text {MOI }}=\overbrace{\left(k_{h i}^{A t t} / k_{h i}^{E q}\right) V_{h i}^{A t t}}^{\begin{array}{c}
\text { disociation from } \\
\text { high affinity sites }
\end{array}}+\overbrace{\left(k_{l o}^{A t t} / k_{l o}^{e q}\right) V_{l o}^{A t t}}^{\begin{array}{c}
\text { disociation from } \\
\text { low affinity sites }
\end{array}} \\
& -\overbrace{\left(k_{h i}^{A t t}\left(B_{h i}^{t o t}-V_{h i}^{A t t}\right)\right.}^{\begin{array}{c}
\text { rate attachment to free } \\
\text { high affinity sites }
\end{array}}+\overbrace{\left.k_{l o}^{A t t}\left(B_{l o}^{t o t}-V_{l o}^{A t t}\right)\right)}^{\begin{array}{c}
\text { rate attachment to free } \\
\text { low affinity sites }
\end{array}} V^{E x}
\end{aligned}
$$

virions attached to low affinity binding sites

$$
\frac{\overbrace{\mathrm{d} V_{l o}^{A t t}}}{\mathrm{~d} t}
$$

virions attached to high affinity binding sites

$$
\frac{\overbrace{\mathrm{d} V_{h i}^{A t t}}^{\mathrm{d} t}}{\text { virions in }}
$$
early endosomes

$$
\frac{\overbrace{\mathrm{d} V^{E n}}^{\mathrm{d} t}}{\mathrm{~d}}
$$$$
\text { vRNPs into }
$$
the cytoplasm $\frac{\overbrace{\mathrm{d} V p^{c y t}}^{\mathrm{d} t}}{\frac{\mathrm{d}}{\mathrm{d}}}=$

$$
=\overbrace{k_{l o}^{A t t}\left(B_{l o}^{t o t}-V_{l o}^{A t t}\right) V^{E x}}^{\begin{array}{c}
\text { attachment to the } \\
\text { free low affinity sites }
\end{array}}
$$

dissociation or endocytosis* low (*same for high \& low)

$$
=\overbrace{k_{h i}^{A t t}\left(B_{h i}^{t o t}-V_{h i}^{A t t}\right) V^{E x}}^{\begin{array}{c}
\text { attachment to the } \\
\text { free high affinity sites }
\end{array}}-\overbrace{\left(k_{h i}^{A t t} / k_{h i}^{e q}+k^{E n}\right) V_{h i}^{A t t}}^{\begin{array}{c}
\text { dissociation or endocytosis* high } \\
\text { (*same for high \& low) }
\end{array}}
$$

$$
=\overbrace{k^{E n}\left(V_{h i}^{A t t}+V_{l o}^{A t t}\right)}^{\begin{array}{c}
\text { rate of } \\
\text { endocytosis }
\end{array}}-\overbrace{\left(k^{F u s}+\frac{1-F_{F u s}}{F_{F u s}} k^{F u s}\right) V^{E n}}^{\begin{array}{c}
\text { fusion of virions in endosomes \& } \\
\text { degradation of virions in lysosomes }
\end{array}}
$$

set of 8 vRNPs released rate entering in into the cytoplasm the nucleus

$$
\overbrace{8 k^{F u s} V^{E n}}
$$$$
-\overbrace{k^{I m p} V p^{c y t}}
$$

$\mathrm{Vp}^{\text {nuc }}$ produces cRNA, $R^{C}$, at a synthesis rate of $k_{C}^{S y n}$, and degraded at a rate of $k_{R}^{D e g}$. Complexes of the viral polymerase, $P_{R d R p}$, and cRNA, $R_{R d R p}^{C}$, associate at the binding rate of $k_{R d R p}^{B i n d}$, where cRNA degrades at a rate of $k_{R}^{D e g} \cdot \mathrm{cRNP}, C p$, is made at the binding rate, $k_{N P}^{B i n d}$, of NP, $P_{N P}$, to $R_{R d R p}^{C}$, which degrades at a rate of $k_{R n p}^{D e g}$ (process of protein production of $P_{N P}$ described later). vRNA $R^{V}$ is synthesized by $C p$ at a rate of $k_{V}^{S y n}$ and degraded at a rate of $k_{R}^{D e g}$. Complexes of the viral polymerase and vRNA are represented by $R_{R d R p}^{V}$, and degraded at a rate of $k_{R R d R p}^{D e g}$ which is lower than that of nascent RNA. Progeny $V p^{n u c}$ is produced by $P_{N P}$ binding to $R_{R d R p}^{V}$. 


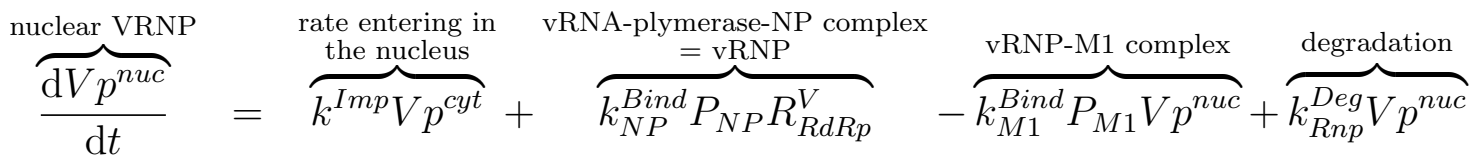

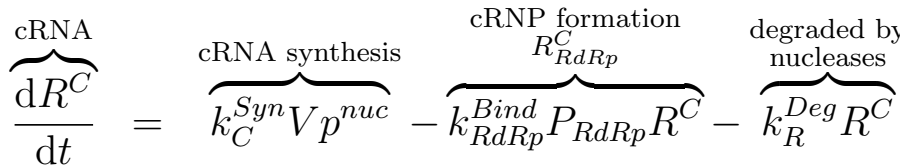

viral polymerase \& cRNA complexes

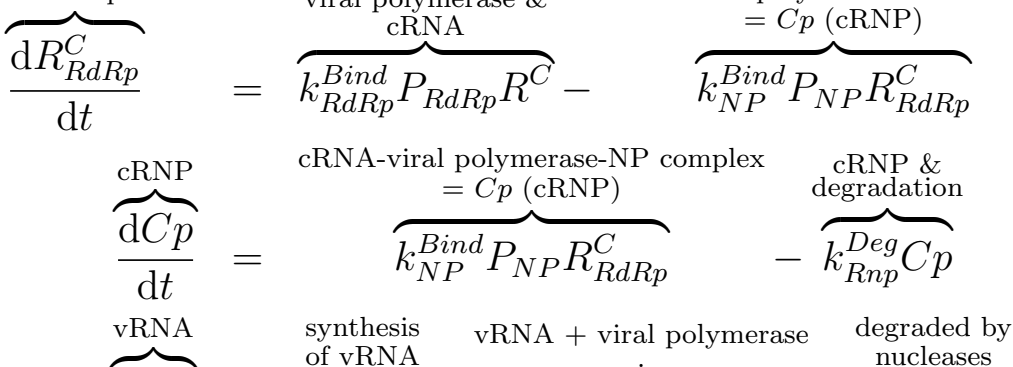

$$
\begin{aligned}
& \overbrace{\frac{\mathrm{d} R^{V}}{\mathrm{~d} t}}=\overbrace{k_{V}^{S y n} C p}^{\text {of vRNA }}-\overbrace{k_{R d R p}^{\text {Bind }} P_{R d R p} R^{V}}^{\text {vin }}-\overbrace{k_{R}^{\text {Deg }} R^{V}}^{\text {nucleases }}
\end{aligned}
$$

viral polymerase \& vRNA complex

$$
\begin{aligned}
& \overbrace{}^{\mathrm{vRNA}}+\text { viral polymerase } \quad \underset{\text { vRNA-viral }}{\text { polymerase-NP complex }} \begin{array}{c}
\text { Rvrr degradation } \\
=\mathrm{vRNP}
\end{array} \\
& \overbrace{\frac{\mathrm{d} R_{R d R p}^{V}}{\mathrm{~d} t}}^{V}=\overbrace{k_{R d R p}^{\text {Bind } P_{R d R p} R^{V}}}^{\overbrace{k_{N P}^{B i n d} P_{N P} R_{R d R p}^{V}}}-\overbrace{k_{R R d R p}^{D e g} R_{R d R p}^{V}}^{\text {Rvrr degradation }}
\end{aligned}
$$

vRNP-M1 complex

$$
\begin{aligned}
& \text { in nucleus } \quad \text { vRNP-M1 } \quad \text { vRNP-NP-M1-NEP complex being }
\end{aligned}
$$

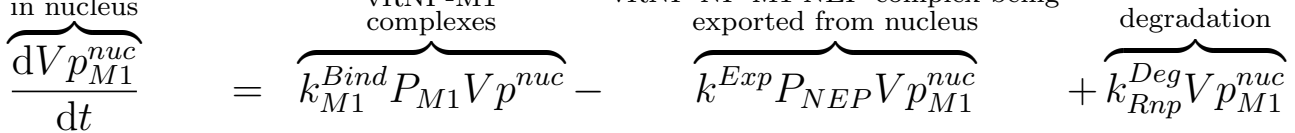

Cytoplasmic

NEP-M1-vRNP complexes

$$
\overbrace{\frac{\mathrm{d} V p_{M 1}^{c y t}}{\mathrm{~d} t}}^{=}
$$

vRNP-NP-M1-NEP complex being exported from nucleus

virus release decay

$=\overbrace{k^{E x p} P_{N E P} V p_{M 1}^{n u c}}-\overbrace{8 r_{R e l}^{R e l}}-\overbrace{k_{R n p}^{D e g} V p_{M 1}^{c y t}}^{\text {in }}$

$\mathrm{Vp}^{\text {nuc }}$ produces mRNA and later protein, where generally $L_{i}$ is the length of an mRNA segment resulting in mRNA, $R_{i}^{M}$, where $i$ is a specific segment. mRNA is degraded at a rate of $k_{M}^{D e g}$ and produced at a rate of $k_{M}^{S y n}$. mRNA is used as a template for protein synthesis, at a rate of $k_{P}^{S y n}$, adjusted by the number of ribosomes which can fit on each segment length i.e. the nucleotide distance between two adjacent ribosomes on an mRNA, of $\mathrm{D}_{\text {Rib }}$. 


$$
\begin{aligned}
& \frac{\mathrm{d} R_{i}^{M}}{\mathrm{~d} t}=\frac{k_{M}^{S y n}}{L_{i}} \frac{V p^{n u c}}{8}-k_{M}^{D e g} R_{i}^{M}, i=1, \ldots, 8 \\
& \frac{\mathrm{d} P_{P B 1}}{\mathrm{~d} t}=\frac{k_{P}^{S y n}}{D_{R i b}} R_{2}^{M}-k^{R d R p} P_{p B 1} P_{p B 2} P_{p A} \\
& \frac{\mathrm{d} P_{P B 2}}{\mathrm{~d} t}=\frac{k_{P}^{S y n}}{D_{R i b}} R_{1}^{M}-k^{R d R p} P_{p B 1} P_{p B 2} P_{p A} \\
& \frac{\mathrm{d} P_{P A}}{\mathrm{~d} t}=\frac{k_{P}^{S y n}}{D_{R i b}} R_{3}^{M}-k^{R d R p} P_{p B 1} P_{p B 2} P_{p A} \\
& \frac{\mathrm{d} P_{R d R p}}{\mathrm{~d} t}=k^{R d R p} P_{p B 1} P_{p B 2} P_{p A}-k_{R d R p}^{B i n d} P_{R d R p}\left(R^{V}+R^{C}\right)-\left(N_{P_{R d R p}}-8\right) r^{R e l} \\
& \frac{\mathrm{d} P_{N P}}{\mathrm{~d} t}=\frac{k_{P}^{S y n}}{D_{R i b}} R_{5}^{M}-\frac{L_{V}}{N_{N P}^{N u c}} k_{N P}^{B i n d} P_{N P}\left(R_{R d R p}^{V}+R_{R d R p}^{C}\right)-\left(N_{P_{N P}}-8 \frac{L_{V}}{N_{N P}^{N u c}}\right) r^{R e l} \\
& \frac{\mathrm{d} P_{M 1}}{\mathrm{~d} t}=\frac{k_{P}^{S y n}}{D_{R i b}}\left(1-F_{S p 17}\right) R_{7}^{M}-\frac{L_{V}}{N_{N P}^{N u c}} k_{M 1}^{\text {Bind }} P_{M 1} V p^{N u c}-\left(N_{P_{M 1}}-8 \frac{L_{V}}{N_{M 1}^{N u c}}\right) r^{R e l} \\
& \frac{\mathrm{d} P_{N E P}}{\mathrm{~d} t}=\frac{k_{P}^{S y n}}{D_{R i b}} F_{S p 18} R_{8}^{M}-\frac{L_{V}}{N_{N E P}^{N u c}} k^{E x p} P_{N E P} V p_{M 1}^{n u c}-\left(N_{P_{N E P}}-8 \frac{L_{V}}{N_{N E P}^{N u c}}\right) r^{R e l} \\
& \frac{\mathrm{d} P_{H A}}{\mathrm{~d} t}=\frac{k_{P}^{S y n}}{D_{R i b}} R_{4}^{M}-N_{P_{H A}} r^{R e l} \\
& \frac{\mathrm{d} P_{N A}}{\mathrm{~d} t}=\frac{k_{P}^{S y n}}{D_{R i b}} R_{6}^{M}-N_{P_{N A}} r^{R e l} \\
& \frac{\mathrm{d} P_{M 2}}{\mathrm{~d} t}=\frac{k_{P}^{S y n}}{D_{R i b}} F_{S p 17} R_{7}^{M}-N_{P_{M 2}} r^{R e l} \\
& \frac{\mathrm{d} V^{R e l}}{\mathrm{~d} t}=r^{R e l}=k^{R e l} V p_{M 1}^{c y t} \prod_{j} \frac{P_{j}}{P_{j}+K_{V_{\text {Rel }} N_{P_{j}}}}
\end{aligned}
$$

where the formation of a complex containing genome segments and sufficient protein is:

$$
\begin{aligned}
= & \prod \frac{P_{R d R p}}{P_{R d R p}+K_{V^{R e l}} N_{P_{R d R p}}} \frac{P_{H A}}{P_{H A}+K_{V \text { Rel }} N_{P_{H A}}} \frac{P_{N P}}{P_{N P}+K_{V R e l} N_{P_{N P}}} \\
& \frac{P_{N A}}{P_{N A}+K_{V^{R e l}} N_{P_{N A}}} \frac{P_{M 1}+K_{V^{R e l}} N_{P_{M 1}}}{P_{M 2}+K_{V^{R e l}} N_{P_{M 2}}} \frac{P_{N E P}}{P_{N E P}+K_{V^{R e l}} N_{P_{N E P}}}
\end{aligned}
$$


The viral polymerase, $P_{R d R p}$, is comprised of three subunits PB1, PB2 and PA ( $P_{P B 1}, P_{P B 2}$ and $P_{P A}$ respectively), which are assumed to be rapidly formed. $P_{R d R p}$ is used in the production of cRNA, $R_{R d R p}^{C}$, and vRNA, $R_{R d R p}^{V}$, at rates of $k_{R d R p}^{B i n d}$, which are both degraded at a rate of $k_{R R d R p}^{D e g} . \quad P_{N P}$ is required for stabilization by both cRNP and vRNP at amounts of, $N_{N P}^{N u c}$, equalling $1 \mathrm{NP}$ molecule bound per 24 nucleotides. Late in infection $V p^{n u c}\left(\right.$ i.e. $\left.R_{R d R p}^{V}+P_{P B 2}\right)$ is bound by $P_{M 1}$ at a rate of $k_{M 1}^{B i n d}$, represented by $V p_{M 1}^{n u c}$. Nuclear export is represented by $k^{E x p}$ and is the combined rate of NEP binding $\left(P_{N E P}\right)$ to M1-vRNP complexes and subsequent transport out of the nucleus of $V p_{M 1}^{c y t}$. $F_{S p 17}$ represents the fraction of spliced M2 mRNAs compared to total mRNAs of segment 7 , and $F_{S p 18}$ is the fraction of spliced NEP mRNAs compared to total mRNAs of segment 8, see Table 2.1.

The remainder of the proteins $\left(P_{H A}, P_{N A}\right.$ and $\left.P_{M 2}\right)$ are used at a rate of $k^{R e l}$, the release rate of progeny virions from the cell which, is proportional to the amount of $V p_{M 1}^{c y t}$. The release of virions, including all steps of transport to the cell membrane and budding, is accounted for in $V^{R e l}$. Sufficient proteins are required for the formation of a virion. $K_{V^{R e l}}$ is the amount of proteins and RNA for this number of complete virions must be present in the cell to reach half the maximum release rate, which is dependant upon the abundance of each protein $P_{j}$ required for one virion $N_{P_{j}}$, of type $j$. 
Table 2.1: Summary of Heldt et al.'s variable naming and description.

\begin{tabular}{ll}
\hline Heldt et al.'s parameters & descrption \\
\hline$V^{E x}$ & The number of virions in the extracellular medium, MOI \\
$V_{l o}^{A t t}$ & The number of virions attached to the binding sites of low affinity \\
$V_{h i}^{A t t}$ & The number of virions attached to the binding sites of high affinity \\
$V^{E n}$ & The number of virions in the early endosome \\
$V p^{c y t}$ & vRNPs (viral ribonucleoproteins) 8 released into the cytoplasm). \\
$V p^{n u c}$ & Nuclear vRNPs which then synthesize mRNA and cRNA (Rc). \\
$R^{C}$ & cRNA synthesized by VPn at a rate ksc. \\
$R^{V}$ & vRNA (viral ribonucleoproteins)made from CP's at rate ksv. \\
$R_{R d R p}^{C}$ & The complex formed by prr and Rc \\
$R_{R d R p}^{V}$ & The complex formed by prr and Rv \\
$C_{p}$ & A synthesized replicative intermediate, second positive-strand full \\
& length RNA (cRNP's) \\
$V_{M 1}^{n u c}$ & Combination of VPn+m1, occurs during the late phase of infec- \\
& tion. Forms at rate kbm1. \\
$V p_{M 1}^{c y t}$ & Combination of VPn+Pm1+pnep, nuclear export rate part pf ke. \\
$R_{1}^{M}$ & The numbers of PB2 mRNA segments \\
$R_{2}^{M}$ & The numbers of PB1 mRNA segments \\
$R_{3}^{M}$ & The numbers of PA mRNA segments \\
$R_{4}^{M}$ & The numbers of HA mRNA segments \\
$R_{5}^{M}$ & The numbers of NP mRNA segments \\
$R_{6}^{M}$ & The numbers of NA mRNA segments \\
$R_{7}^{M}$ & The numbers of M1 and M2 mRNA segments \\
$R_{8}^{M}$ & The numbers of NEP mRNA segments \\
$P_{H A}$ & The number of PA Proteins \\
$P_{M 1}$ & The number of M1 protein \\
$P_{M 2}$ & The number of M2 Proteins \\
$P_{N A}$ & The number of NA Proteins \\
$P_{N E P}$ & The number of NEP Proteins, facilitate the nuclear export. \\
$P_{N P}$ & The number of NP Proteins. NP leads to cRNP and vRNP. \\
$P_{p B 1}$ & The number of PB1 Proteins \\
$P_{p B 2}$ & The number of PA Proteins \\
$P_{p A}$ & \\
$P_{R d R p}$ & \\
& The number of new viral polymerase. Both CP and Rv bind to it.
\end{tabular}


Table 2.2: Summary of Heldt et al.'s parameter naming and description.

\begin{tabular}{|c|c|c|}
\hline $\begin{array}{l}\text { Heldt et al.'s } \\
\text { best fit }\end{array}$ & value & description \\
\hline$B_{h i}$ & 150 sites & Free binding sites of high affinity \\
\hline$B_{l o}$ & 1000 sites & Free binding sites of low affinity \\
\hline$D_{R i b}$ & $160 \mathrm{nt}$ & Distance between two adjacent ribosomes \\
\hline$k_{h i}^{A t t}$ & 0.0809 sites $^{-1} \cdot \mathrm{h}^{-1}$ & $\begin{array}{l}\text { The rate at which virions attach to free high } \\
\text { affinity binding sites }\end{array}$ \\
\hline$k_{l o}^{A t t}$ & $4.55 \cdot 10^{-4}$ & $\begin{array}{l}\text { The rate at which virions attach to free low } \\
\text { affinty binding sites }\end{array}$ \\
\hline$k_{M 1}^{B i n d}$ & $1.39 \cdot 10^{-6}$ molecules $^{-1} \cdot \mathrm{h}^{-1}$ & The rate at which VPn binds to Pm1 \\
\hline$k_{N P}^{B \text { Bind }}$ & $3.01 \cdot 10^{-4}$ molecules $^{-1} \cdot \mathrm{h}^{-1}$ & Binding of NP to RdRp-RNA complexes \\
\hline$k_{R d R p}^{\text {Bind }}$ & 1 molecule $^{-1} \cdot \mathrm{h}^{-1}$ & Binding of RdRp complexes to RNA \\
\hline$k_{M}^{D e g}$ & $0.33 \mathrm{~h}^{-1}$ & Degradation of mRNA \\
\hline$k_{R}^{D e g}$ & $36.36 \mathrm{~h}^{-1}$ & $\begin{array}{l}\text { The rate of degradation of both } \mathrm{CP} \text { and } \\
\text { vRNA }\end{array}$ \\
\hline$k_{R n p}^{D e g}$ & $0.09 \mathrm{~h}^{-1}$ & $\begin{array}{l}\text { The rate of degradation of } c R N P(C P) \text { and } \\
\text { vRNP (Vpn). }\end{array}$ \\
\hline$k_{R R d R p}^{D e g}$ & $4.25 \mathrm{~h}^{-1}$ & $\begin{array}{l}\text { The rate of degradation of both Rcrr and } \\
\text { Rvrr }\end{array}$ \\
\hline$k^{E x p}$ & $1 \cdot 10^{-6}$ molecules $\cdot \mathrm{h}^{-1}$ & NEP binding and nuclear export \\
\hline$K^{E n}$ & $4.8 \mathrm{~h}^{-1}$ & $\begin{array}{l}\text { Rate of virion entering the cell through en- } \\
\text { docytosis }\end{array}$ \\
\hline$k_{h i}^{E q}$ & 0.0113 site $^{-1}$ & Equilibrium constant of high-affinity sites \\
\hline$k_{l o}^{E q}$ & $8.33 \cdot 10^{-5} \mathrm{site}^{-1}$ & $\begin{array}{l}\text { Equilibrium constant of low-affinity binding } \\
\text { sites }\end{array}$ \\
\hline$k^{F u s}$ & $3.21 \mathrm{~h}^{-1}$ & $\begin{array}{l}\text { The rate at which fusion of virions in endo- } \\
\text { somes occurs }\end{array}$ \\
\hline$k^{\operatorname{Imp} p}$ & $6 \mathrm{~h}^{-1}$ & $\begin{array}{l}\text { Rate that VPc's enter into nucleus, where } \\
\text { they becomes VPn }\end{array}$ \\
\hline$k^{r e l}$ & $\begin{array}{l}3.7 \cdot 10^{-3} \\
\text { virions } \cdot \text { molecules }^{-1} \cdot \mathrm{h}^{-1}\end{array}$ & Virus release rate \\
\hline$k^{R d R p}$ & 1 molecule $^{-2} \cdot \mathrm{h}^{-1}$ & Formation of RdRp complexes \\
\hline$k_{C}^{S y n}$ & $1.38 \mathrm{~h}^{-1}$ & cRNA synthesis \\
\hline$k_{M}^{\text {Syn }}$ & $2.5 \cdot 10^{-5} \cdot-1$ & mRNA synthesis \\
\hline$k_{P}^{\text {Syn }}$ & $64,800 \mathrm{nt} \cdot \mathrm{h}^{-1}$ & Protein synthesis \\
\hline$k_{V}^{S y n}$ & $13.86 \mathrm{~h}^{-1}$ & The rate of $R v$ production. \\
\hline$K_{V} R e l$ & 10 & $\begin{array}{l}\text { Influence of proteins on virus release (ad- } \\
\text { justed) }\end{array}$ \\
\hline$F_{F u s}$ & 0.51 & Fraction of fusion competent virions \\
\hline$F_{S p 17}$ & 0.02 & Fraction of M2-encoding mRNAs \\
\hline$F_{S p 18}$ & 0.125 & Fraction of NEP-encoding mRNAs \\
\hline
\end{tabular}


Table 2.3: Summary of Heldt et al.'s parameter naming and description (continuation)

\begin{tabular}{|c|c|c|}
\hline $\begin{array}{l}\text { Heldt et al.'s best } \\
\text { fit parameter }\end{array}$ & value & description \\
\hline$L_{i}$ & $\mathrm{i}$ & Length of mRNA segment \\
\hline$L_{i}$ & $1757 \mathrm{nt}$ & Length of segment HA mRNA \\
\hline$L_{i}$ & $1005 \mathrm{nt}$ & Length of segment M1 \& M2 mRNA \\
\hline$L_{i}$ & $1392 \mathrm{nt}$ & Length of segment NA mRNA \\
\hline$L_{i}$ & $868 \mathrm{nt}$ & Length of segment NEP mRNA \\
\hline$L_{i}$ & $1540 \mathrm{nt}$ & Length of segment NP mRNA \\
\hline$L_{i}$ & $2211 \mathrm{nt}$ & Length of segment PA mRNA \\
\hline$L_{i}$ & $2320 \mathrm{nt}$ & Length of segment PB1 mRNA \\
\hline$L_{i}$ & $2320 \mathrm{nt}$ & Length of segment PB2 mRNA \\
\hline$L_{V}$ & $1700 \mathrm{nt}$ & Average length of a vRNA \\
\hline$N_{M 1}^{N u c}$ & $200 \mathrm{nt}$ & Nucleotides bound by one M1 molecule \\
\hline$N_{N E P}^{N u c}$ & $1700 \mathrm{nt}$ & Nucleotides bound by one NEP molecule \\
\hline$N_{N P}^{N u c}$ & $24 \mathrm{nt}$ & Nucleotides bound by one NP molecule \\
\hline$N_{P_{H A}}$ & 500 molecules $\cdot$ virion $^{-1}$ & Number of HA molecules in a virion \\
\hline$N_{P_{M 1}}$ & 3000 molecules $\cdot$ virion $^{-1}$ & Number of M1 molecules in a virion \\
\hline$N_{P_{M 2}}$ & 40 molecules $\cdot$ virion $^{-1}$ & Number of M2 molecules in a virion \\
\hline$N_{P_{N A}}$ & 100 molecules $\cdot$ virion $^{-1}$ & Number of NA molecules in a virion \\
\hline$N_{P_{N E P}}$ & 165 molecules $\cdot$ virion $^{-1}$ & Number of NEP molecules in a virion \\
\hline$N_{P_{N P}}$ & 1000 molecules $\cdot$ virion $^{-1}$ & Number of NP molecules in a virion \\
\hline$N_{P_{R d R p}}$ & 45 molecules $\cdot$ virion $^{-1}$ & Number of RdRp complexes in a virion \\
\hline$r^{\text {Rel }}$ & - & $\begin{array}{l}\text { The overall rate of virus release. Propor- } \\
\text { tional the amount of cytoplasmic vRNPs } \\
\text { and to } \mathrm{kr}\end{array}$ \\
\hline
\end{tabular}

Heldt et al.'s MM has 32 variables and 48 parameters. The values from Heldt et al.'s MM, Table 2.2 and 2.3, are used at starting guesses for fitting to experimental data. Our MM, described hereafter is visually depicted in Figure 2.1. 


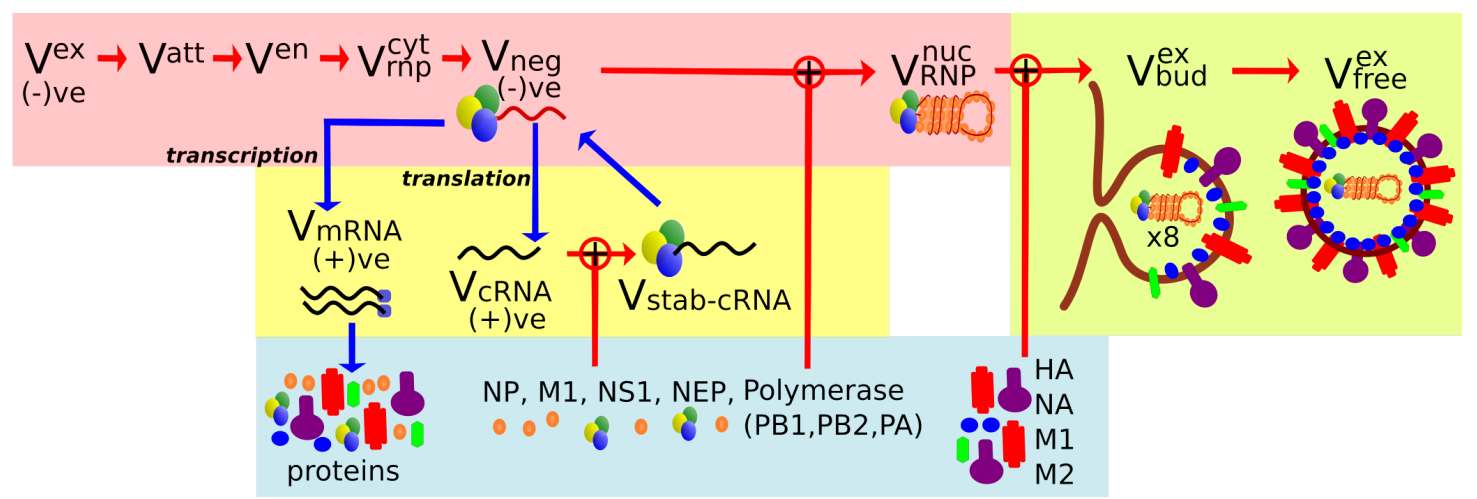

Figure 2.1: Diagram of intracellular IAV infection. Depiction of the replication steps as represented in the MM, showing the progression of replication and indicating which steps require viral proteins in order to proceed.

Extracellular virions, $V^{\mathrm{Ex}}$, represent the virions used to infect cells, which attach to receptors on the cell surface at a rate of $k^{\text {att }}$, and detaches at a rate of $k^{\text {det }}$. The available number of receptors is the difference between the number of total sites $N^{\text {sites }}$ and those occupied by attached virions $V^{\text {att }}$. Virions are cleared at a rate of c at stages prior to endocytosis, $V^{\mathrm{En}}$, which occurs at a rate of $k^{\mathrm{en}}$.

The viral segments in the nucleus are used as templates to produce viral mRNA, $V^{\mathrm{mRNA}}$, at a rate of $r_{\mathrm{mRNA}}^{\mathrm{prod}} \cdot V^{\text {neg }}$ and $V^{\mathrm{mRNA}}$ are degraded by $r_{\mathrm{vRNA}}^{\mathrm{deg}}$ and $r_{\mathrm{mRNA}}^{\mathrm{deg}}$ respectively. Assuming that $N_{\text {rib }}$ can be on one average length mRNA at a time, protein is translated at a rate of $k_{\text {pro }}^{\text {synth }}$. Protein is sub-categorised by those which require import back into the nucleus, Protein, and those transported to the membrane, Protein ${ }^{\mathrm{Memb}}$, at a rate of $k_{\text {pro }}^{\text {memb }}$.

The import of proteins into the nucleus then allows for the production of stabilized cRNA, $V^{\text {stab-cRNA }}$, non-stabilized cRNA, $V^{\text {cRNA }}$. It has been shown that non-stabilized cRNA, is always produced during infection at a rate of $k_{\mathrm{cRNA}}^{\mathrm{prod}}$, by $V^{\text {neg }}$, however is rapidly degraded at rate, $r_{\text {cRNA }}^{\text {deg }}$, when insufficient proteins are available. Proteins are required to stabilize cRNA, at a rate of $k_{\mathrm{cRNA}}^{\mathrm{stab}}$, dependant upon there being sufficient protein to satisfy that $N_{\text {pro }}^{\text {stab }}$ will be used per stabilized cRNA produced. $V^{\text {stab-cRNA }}$ is degraded at a rate of $r_{\text {cRNA }}^{\text {deg }}$, and produces progeny, $V^{\text {neg }}$, at a rate of $k_{\text {stab-cRNA }}^{\text {progeny-prod }}$. 
$V^{\text {neg }}$ is assembled into $V$ rnp ${ }^{\text {nucleus }}$, at a rate of $k_{\text {vRNP }}^{\text {assembly }}$, with the number of proteins, $N_{\mathrm{vRNP}}^{\mathrm{prot}}$, being lost per vRNP produced. vRNP are then degraded at a rate of $r_{\mathrm{vRNP}}^{\mathrm{deg}}$ or exported and packaged at a rate of $r_{\mathrm{vRNP}}^{\mathrm{pack}}$ into $V_{\mathrm{ex}}^{\text {bud }}$. Once budded, the progeny virion is released at a rate of $r_{\mathrm{vRNP}}^{\text {release }}$ to $V_{\mathrm{ex}}^{\text {free }}$. Both budding virions, and released virions are cleared at a rate $\mathrm{c}$.

Our simplified intracellular IAV mathematical model is given by:

$$
\begin{aligned}
& \frac{\mathrm{d} V^{\mathrm{Ex}}}{\mathrm{d} t}=k^{\mathrm{det}} V^{\mathrm{att}}-k^{\mathrm{att}}\left(1-V^{\mathrm{att}} / N^{\mathrm{sites}}\right) V^{\mathrm{Ex}}-c V^{\mathrm{Ex}} \\
& \frac{\mathrm{d} V^{\mathrm{att}}}{\mathrm{d} t}=k^{\mathrm{att}}\left(1-V^{\mathrm{att}} / N^{\mathrm{sites}}\right) V^{\mathrm{ex}}-k^{\mathrm{det}} V^{\mathrm{att}}-k^{\mathrm{en}} V^{\mathrm{att}}-c V^{\mathrm{Att}} \\
& \frac{\mathrm{d} V^{\mathrm{En}}}{\mathrm{d} t}=k^{\mathrm{en}} V^{\text {att }}-k^{\text {fusion }} V^{\mathrm{en}} \\
& \frac{\mathrm{d} V \mathrm{rnp}^{\mathrm{cyt}}}{\mathrm{d} t}=8 k^{\text {fusion }} k_{\text {fusion }}^{\text {fract }} V^{\mathrm{en}}-k_{\mathrm{seg}}^{\mathrm{imp}} V \mathrm{rnp}^{\mathrm{cyt}} \\
& \frac{\mathrm{d} V^{\text {neg }}}{\mathrm{d} t}=k_{\mathrm{seg}}^{\mathrm{imp}} V \operatorname{rnp}^{c y t}+k_{\text {stab-cRNA }}^{\text {progeny-prod }} V^{\text {stab-cRNA }}-k_{\text {vRNP }}^{\text {assembly }} V^{\text {neg }} \frac{\text { Protein }}{N_{\text {vRNP }}^{\text {prot }}}-r_{\text {vRNA }}^{\text {deg }} V^{\text {neg }} \\
& \frac{\mathrm{d} V^{\mathrm{mRNA}}}{\mathrm{d} t}=r_{\mathrm{mRNA}}^{\mathrm{prod}} V^{\mathrm{neg}}-r_{\mathrm{mRNA}}^{\mathrm{deg}} V^{\mathrm{mRNA}} \\
& \frac{\mathrm{dProtein}}{\mathrm{d} t}=N_{\text {rib }} k_{\text {pro }}^{\text {synth }} \frac{5.5}{8} V^{\mathrm{mRNA}}-k_{\text {cRNA }}^{\text {stab }} V^{\text {cRNA }} \text { Protein }-k_{\text {vRNP }}^{\text {assembly }} V^{\text {neg }} \text { Protein } \\
& \frac{\text { dProtein }^{\text {Memb }}}{\mathrm{d} t}=N_{\text {rib }} k_{\text {pro }}^{\text {synth }} \frac{2.5}{8} V^{\text {mRNA }}-k_{\text {pro }}^{\text {memb }} \operatorname{Protein}^{\text {Memb }} \\
& \frac{\mathrm{d} V^{\mathrm{cRNA}}}{\mathrm{d} t}=k_{\mathrm{cRNA}}^{\mathrm{prod}} V^{\mathrm{neg}}-k_{\mathrm{stab} \mathrm{cRNA}}^{\mathrm{prod}} V^{\mathrm{cRNA}} \frac{\text { Protein }}{N_{\text {pro }}^{\text {stab }}}-r_{\mathrm{cRNA}}^{\mathrm{deg}} V^{\mathrm{cRNA}} \\
& \frac{\mathrm{d} V^{\text {stab-cRNA }}}{\mathrm{d} t}=k_{\text {stab cRNA }}^{\text {prod }} V^{\text {cRNA }} \frac{\text { Protein }}{N_{\text {pro }}^{\text {stab }}}-r_{\text {cRNA }}^{\text {deg }} V^{\text {stab-cRNA }} \\
& \frac{\mathrm{d} V \mathrm{rnp}^{\text {nucleus }}}{\mathrm{d} t}=k_{\mathrm{vRNP}}^{\text {assembly }} V^{\text {neg }} \frac{\text { Protein }}{N_{\mathrm{vRNP}}^{\text {prot }}}-r_{\mathrm{vRNP}}^{\text {pack } \operatorname{Vrnp}}{ }^{\text {nucleus }}-r_{\mathrm{vRNP}}^{\text {deg } \operatorname{Vrnp}}{ }^{\text {nucleus }} \\
& \frac{\mathrm{d} V_{\mathrm{ex}}^{\text {bud }}}{\mathrm{d} t}=\frac{1}{8} r_{\mathrm{vRNP}}^{\text {pack }} V \mathrm{rnp}^{\text {nucleus }}-r_{\mathrm{vRNP}}^{\text {release }} V_{\mathrm{ex}}^{\text {bud }}-c V_{\mathrm{ex}}^{\text {bud }} \\
& \frac{\mathrm{d} V_{\mathrm{ex}}^{\text {free }}}{\mathrm{d} t}=r_{\mathrm{vRNP}}^{\text {release }} V_{\mathrm{ex}}^{\text {bud }}-c V_{\mathrm{ex}}^{\text {free }}
\end{aligned}
$$


The values for the MM parameters are reported in Table 2.6, with the data set used to determine those values denoted where applicable. Our MM has 13 variables and 25 parameters, as compared to the MM by Heldt et al. which, has 25 variables and 48 parameters.

Table 2.4: Summary of our variable naming and description.

\begin{tabular}{|c|c|}
\hline Our parameters & description \\
\hline$V^{E x}$ & The number of virions in the extracellular medium, MOI \\
\hline$V^{A t t}$ & $\begin{array}{l}\text { The number of virions attached to the binding sites of low and } \\
\text { high affinity }\end{array}$ \\
\hline$V^{E n}$ & The number of virions in the early endosome \\
\hline Vrnp $p^{c y t}$ & vRNPs (viral ribonucleoproteins) 8 released into the cytoplasm. \\
\hline$V^{\text {neg }}$ & $\begin{array}{l}\text { Nuclear vRNPs which then synthesize mRNA and cRNA and } \\
\text { progeny vRNP (vRNA-NP-PB1-PB2-PA). }\end{array}$ \\
\hline$V^{m R N A}$ & mRNAs produced from $V^{n e g}$ \\
\hline Protein & $\begin{array}{l}\text { The number of proteins represents the segments imported into the } \\
\text { nucleus for RNA stabilization, } \mathrm{PB} 1, \mathrm{~PB} 2, \mathrm{PA}, \mathrm{NP}, \mathrm{NEP} \text { and } \mathrm{M} 1 \text { ) }\end{array}$ \\
\hline Protein ${ }^{\text {Memb }}$ & $\begin{array}{l}\text { The transmembrane proteins M2, HA and NA transported to the } \\
\text { apical membrane of the cell }\end{array}$ \\
\hline$V^{c R N A}$ & Synthesized replicative intermediate cRNA synthesized by $V^{\text {neg }}$ \\
\hline$V^{s t a b-c R N A}$ & Stabilized cRNA available for production of $V^{n e g}$ \\
\hline Vrnp $p^{\text {nucleus }}$ & Progeny vRNPs (NEP-M1-vRNP complex) \\
\hline$V_{e x}^{b u d}$ & Cytoplasmic virions budding at the surface in a $7+1$ configuration \\
\hline$V_{e x}^{f r e e}$ & Progeny virions \\
\hline
\end{tabular}

Table 2.5: Summary of our degradation rates. These parameter values were determined through MM fitting of Kawakami et al. RNA data.

\begin{tabular}{lll}
\hline Our parameters & value & description \\
\hline$r_{m R N A}^{\text {deg }}$ & $2.28254 \cdot 10^{-1} \mathrm{~h}^{-1}$ & Degradation rate of mRNA \\
$r_{v R N A}^{d e g}$ & $26452.6 \mathrm{~h}^{-1}$ & Degradation rate of vRNA \\
$r_{v R N P}^{d e g}$ & $3.30282 \cdot 10^{-27} \mathrm{~h}^{-1}$ & Degradation rate of vRNP \\
$r_{c R N A}^{\text {deg }}$ & $4.1547 \cdot 10^{-2} \mathrm{~h}^{-2}$ & Degradation rate of cRNA \\
$r_{\text {stabcRNA }}^{\text {deg }}$ & $1.9374 \cdot 10^{-2} \mathrm{~h}^{-1}$ & Degradation rate of stabilized cRNA
\end{tabular}




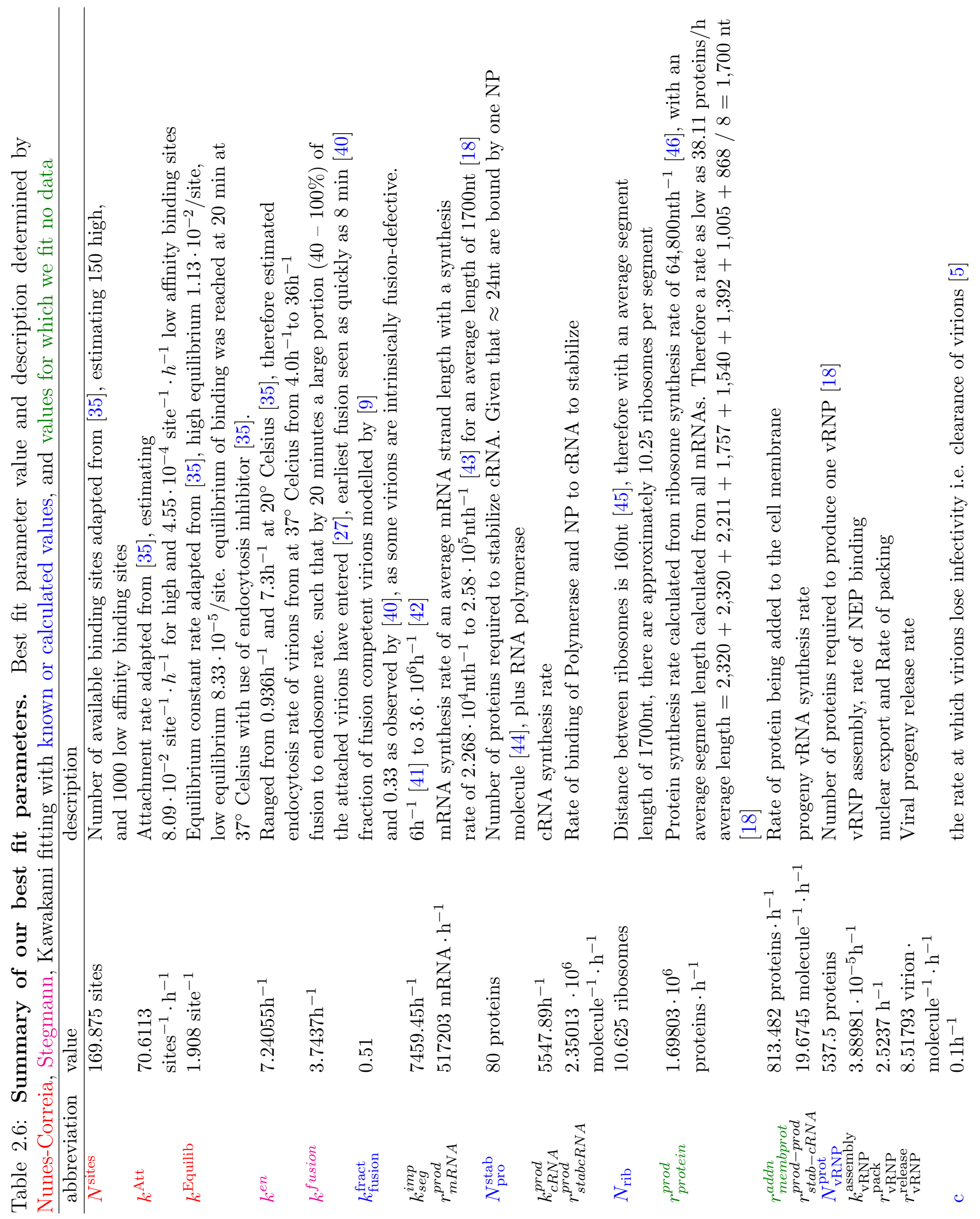




\section{Chapter 3}

\section{Results}

\subsection{Kinetics of attachment of free virions}

We first examine the use of high and low affinity binding sites by IAV for entry into the cell using data published by Nunes-Correia et al., shown in Figure 3.3 [35]. Nunes-Correia et al. studied the virus-cell binding at varying input IAV concentrations to produce scatchard plots of influenza virus binding to MDCK cells. They report attachment via two binding site types, high and low affinity, 150 sites and 1000 sites respectively, as illustrated in 3.1. We represent high and low affinity with one averaged attachment rate and determined MM via two mode entry was an unnecessary complication.

Influenza possesses an attachment protein, hemagglutinin, which binds to the sugar epitope of a receptor i.e., the sialic acid of a glycoprotein on the surface of cells. Nunes-Correia et al. postulate that the different rates are due to attachment of hemagglutinin to its preferred sugar epitope, sialic acid, and attachment via a non-specific, perhaps even non-sialic acid, ligand at a lower affinity [35]. Nycholat et al. illustrated that different hemagglutinin subtypes (or within subtypes) preferred different terminal glycans, which can affect entry rates [1]. Additionally, in a profiling study by Wang et al. it was found that the contribution to bind- 
ing affinity came from both core and terminal sugars on hemagglutinin receptor glycans, but also from hemagglutinin structure glycosylation itself, which is strain specific. They subjected glycans that are normally present on the surface of cells and hemagglutinin to enzymes that cut surface sugars shorter, and through glycan microarray profiling showed increased binding affinity to hemagglutinin. They found that fewer complex groups present on the surface of hemagglutinin and/or the microarray (representing the cell surface) resulted in decreased binding specificity but more overall binding [47]. This illustrates that glycosylation is important for entry of the virus, acting as a internalization co-receptor [1]. As such, there exists an additional level of regulation of binding affinity directly from the glycans.

Hemagglutinin undergoes yearly genetic shift, as such the preference for different receptors is constantly evolving. Thus, the non-specific binding should not be tied down to one value as it can be different depending on the strain and cell type used for testing. If we get too specific, then we run the risk being unable to properly characterize other strains with different specificities. Since it has been shown that IAV can infect a cell even when a cell is subjected to an enzyme that cuts surface sugars, a general entry term can reflect a larger group of interactions. As such, it would be advantageous to not over specify binding for one strain thus excluding the kinetics of different strains.

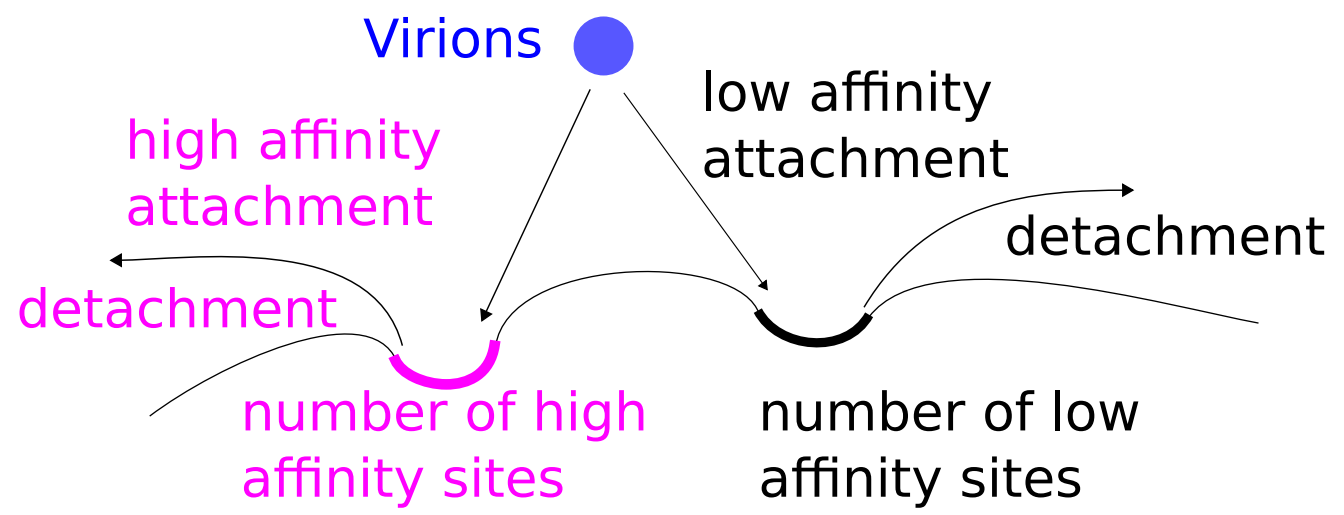

Figure 3.1: Scheme of the influenza virus attachment. Attachment at both high (fuchsia) and low (black) affinity sites. Endocytosis and fusion are assumed to be the same for virions regardless of their attachment site type. 
Heldt et al.'s high and low affinity attachment:

$$
\begin{aligned}
& \overbrace{\frac{\mathrm{d} V^{E x}}{\mathrm{~d} t}}^{\text {MOI }}=\overbrace{\left(k_{h i}^{A t t} / k_{h i}^{E q}\right) V_{h i}^{A t t}}^{\begin{array}{c}
\text { disociation from } \\
\text { high affinity sites }
\end{array}}+\overbrace{\left(k_{l o}^{A t t} / k_{l o}^{e q}\right) V_{l o}^{A t t}}^{\begin{array}{c}
\text { disociation from } \\
\text { low affinity sites }
\end{array}} \\
& -\overbrace{\left(k_{h i}^{A t t}\left(B_{h i}^{t o t}-V_{h i}^{A t t}\right)\right.}^{A}+\overbrace{\left.k_{l o}^{A t t}\left(B_{l o}^{t o t}-V_{l o}^{A t t}\right)\right)} V^{E x}
\end{aligned}
$$

virions attached to low affinity binding sites

$$
\frac{\overbrace{\mathrm{d} V_{l o}^{A t t}}}{\mathrm{~d} t}
$$

virions attached to high affinity binding sites

$$
\frac{\overbrace{\mathrm{d} V_{h i}^{A t t}}^{\mathrm{d} t}}{\mathrm{~d}}
$$

$$
=\overbrace{k_{l o}^{A t t}\left(B_{l o}^{t o t}-V_{l o}^{A t t}\right) V^{E x}}^{\begin{array}{c}
\text { attachment to the } \\
\text { free low affinity sites }
\end{array}}-\overbrace{\left(k_{l o}^{A t t} / k_{l o}^{e q}+k^{E n}\right) V_{l o}^{A t t}}^{\begin{array}{c}
\text { dissociation or endocytosis* low } \\
\text { (*same for high \& low })
\end{array}}
$$$$
\text { dissociation or endocytosis* high }
$$

$$
=\overbrace{k_{h i}^{A t t}\left(B_{h i}^{t o t}-V_{h i}^{A t t}\right) V^{E x}}^{\begin{array}{c}
\text { attachment to the } \\
\text { free high affinity sites }
\end{array}}-\overbrace{\left(k_{h i}^{A t t} / k_{h i}^{e q}+k^{E n}\right) V_{h i}^{A t t}}^{\begin{array}{c}
\text { dissociation or endocytosis* hig } \\
\text { (*same for high \& low })
\end{array}}
$$


Our proposed single attachment:

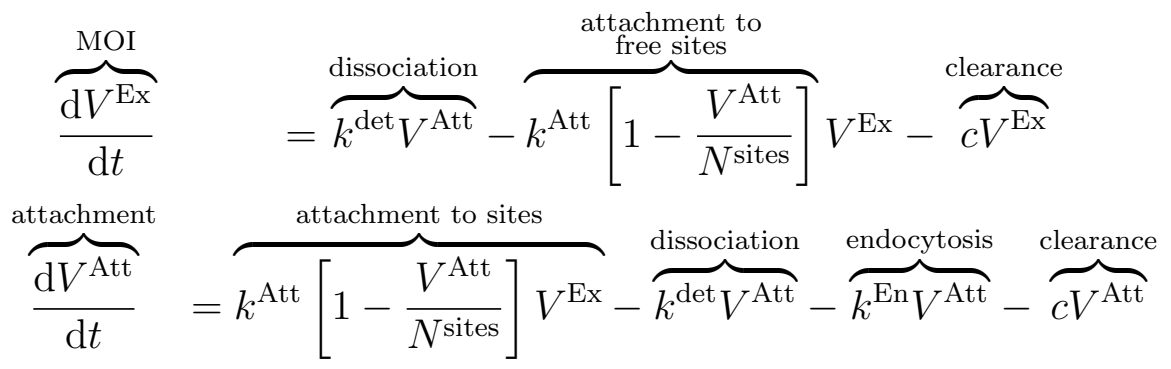

with the following relation:

$$
k^{\text {Equilib }}=k^{A t t} / k^{\text {det }}
$$

In the MM by Heldt et al. two attachment types were considered, low affinity attachment, $V_{l o}^{A t t}$, and high affinity attachment, $V_{h i}^{A t t}$, equivalent to our general attachment term, $V^{\text {Att }}$, a simplification which does not affect the MMs function. Heldt et al.'s MM represents low and high affinity site attachment rates $\left(k_{l o}^{A t t}\right.$ and $\left.k_{h i}^{A t t}\right)$, while our MM represents an averaged attachment rate of free virions $\left(k^{\mathrm{Att}}\right)$. Heldt et al.'s binding equilibrium rates $k_{l o}^{e q}$ and $k_{h i}^{e q}$ are equivalent to our detachment rate, $k^{\text {det}}$, due to the relation shown above. In Heldt et al.'s MM, virions can move between the supernatant, $V^{\mathrm{Ex}}$, and either attachment type continuously, until they result in an infection. A key difference in our MM is the inclusion of a clearance term $c$, to account for viral loss of infectivity. The addition of a clearance term means that a portion of the virions in the extracellular medium, $V^{\mathrm{Ex}}$, can be removed from the system, more alike biological events. 
We ensured that our 1-mode entry could reach binding equilibrium by 20 minutes as seen in Figure 3.2. Heldt et al.'s 2-mode MM reached 95\% attachment equilibrium by 20 minutes, due primarily to the effect of low affinity binding. However, when considering only high affinity binding equilibrium for 2-mode, it is reached shortly after 2 minutes, which can be recapitulated via 1-mode attachment, shown in Figure 3.2. Heldt et al.'s 1 mode MM has 150 and 1000 high and low affinity binding sites respectively, while our 1 mode has $\approx 170$ sites total.

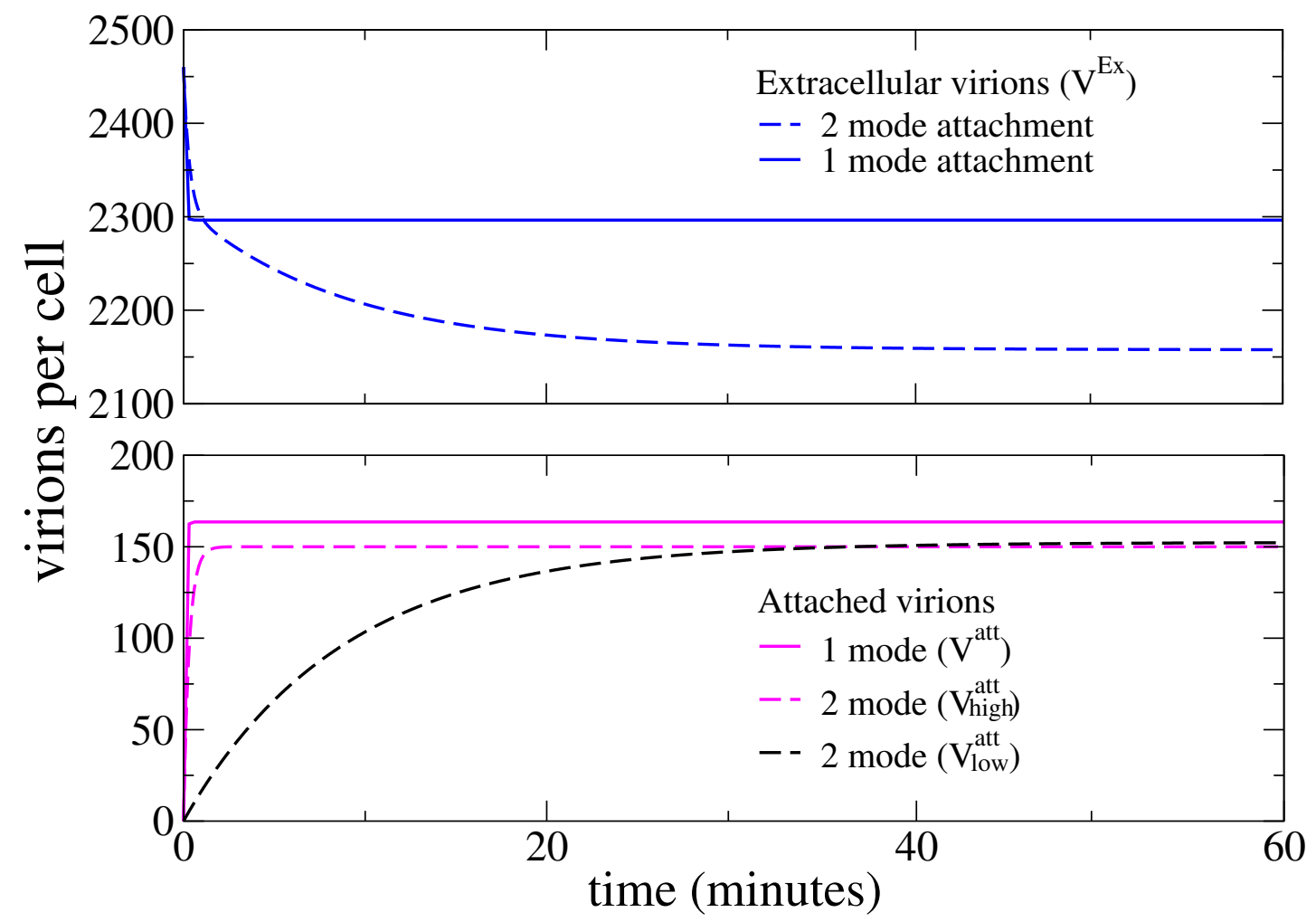

Figure 3.2: Comparison of fusion equilibrium for 1- and 2-mode attachment. Attachment values predicted by the MMs for both Heldt et al.'s 2-mode attachment (dashed) and our 1-mode attachment (solid). The MOI was set to 2460 virions initially attached as to encompass the largest inocula experimentally accounted for by Nunes-Correia et al., see Figure 3.3 for experimental data. 
To determine the relationship between the number of virions added, and the resultant equilibrium levels of free and attached virions after 20 minutes, the following equations were used to solve for $V^{e x}$ and $V^{\text {att }}$ :

$$
\begin{aligned}
0 & =k^{\text {att }}\left(N^{\text {sites }}-V^{\text {att }}\right) V^{e x}-k^{\text {det }} V^{\text {att }} \\
\cdots & =k^{\text {att }}\left(N^{\text {sites }}-V^{\text {att }}\right) V^{e x}-k^{\text {det }} V^{\text {att }} \\
0 & =\frac{k^{\text {det }} V^{\text {att }}}{k^{\text {att }}\left(N^{\text {sites }}-V^{\text {att }}\right)} \\
V^{e x} & \frac{k^{\text {det }}\left(S-V^{\text {ex }}\right)}{k^{\text {att }}\left(N^{\text {sites }}-S+V^{\text {ex }}\right)} \\
V^{e x} & =k^{\text {Equilib }}\left(S-V^{\text {ex }}\right) \\
\left(V^{\text {ex }}\right)^{2}-S V^{\text {ex }}+N^{\text {sites }} V^{\text {ex }} & =k^{\text {Equilib }} S-k^{\text {Equilib }} V^{\text {ex }} \\
0 & =\left(V^{\text {ex }}\right)^{2}+\left(N^{\text {sites }}-S+k^{\text {Equilib }}\right) V^{\text {ex }}-k^{\text {Equilib }} S \\
V^{\text {ex }} & =\frac{-\left(N^{\text {sites }}-S+k^{\text {Equilib }}\right)+\sqrt{\left(N^{\text {sites }}-S+k^{\text {Equilib }}\right)^{2}+4 k^{\text {Equilib } S}}}{2}
\end{aligned}
$$

and

$$
\begin{aligned}
& 0=k^{a t t}\left(N^{\text {sites }}-V^{a t t}\right) V^{e x}-k^{d e t} V^{a t t} \\
& k^{\text {det }} V^{\text {att }}=k^{\text {att }}\left(N^{\text {sites }}-V^{\text {att }}\right) V^{e x} \\
& V^{a t t}=\frac{k^{a t t}}{k^{d e t}}\left(N^{\text {sites }}-V^{a t t}\right)\left(S-V^{a t t}\right) \\
& V^{a t t} \frac{k^{\text {det }}}{k^{\text {att }}}=N^{\text {sites }} S-N^{\text {sites }} V^{a t t}-S V^{\text {att }}+\left(V^{\text {att }}\right)^{2} \\
& 0=\left(V^{\text {att }}\right)^{2}-\left(N^{\text {sites }}+S+k^{\text {Equilib }}\right) V^{\text {att }}+N^{\text {sites }} S \\
& V^{\text {att }}=\frac{\left(N^{\text {sites }}+S+k^{\text {Equilib }}\right)-\sqrt{\left(N^{\text {sites }}+S+k^{\text {Equilib }}\right)^{2}+4 N^{\text {sites }} S}}{2}
\end{aligned}
$$

where

$$
\frac{k^{\text {detach }}}{k^{\text {att }}}=k^{\text {Equilib }}
$$

Where $S$ represents the totals virions in the system (i.e. the inoculum), moving between the supernatant $\left(V^{e x}\right)$ and attachment to cell receptors $\left(V^{\text {att }}\right)$. Based on these expressions we calculate the $k^{\text {att }}, k^{\text {detach }}$ and $N^{\text {att }}$ rates. 
Based on the above equations, the amount of binding sites seems to limit the amount of bound virions. However, this does not match the data from NunesCorreia et al., at high MOIs, which would seem to illustrate that binding increases as MOI increases indefinitely. This suggests that the number of available binding sites is much greater than input virions. These very high MOIs, however, may not be representative of actual infections which are typically initiated with much lower MOIs. As such, we focused on developing the MM to match data for lower MOI data points.

As we no longer explicitly account for two attachment rates, we determined what number of attachment sites accounts for both low and high affinity site in one term, resulting in best representing Nunes-Correia et al. data at low MOIs. To do so, data was simulated while disregarding up to 4 data points from the higher MOIs, as seen in Figure 3.3. Table 3.1 shows the parameter values for the MM fit with all data points used or 1, 2, 3, or 4 of the highest total virion data excluded.

Table 3.1: Best fit parameters for attachment. Fits with all Nunes-Correia et al. free and attached virion data, with incremental exclusion of high MOI points.

\begin{tabular}{lllll}
\hline $\begin{array}{l}\text { data or number } \\
\text { of removed points }\end{array}$ & $\begin{array}{l}\text { number of } \\
\text { attachment sites }\end{array}$ & $\begin{array}{l}\text { attachment } \\
\text { rate }\end{array}$ & $\begin{array}{l}\text { equilibrium } \\
\text { rate }\end{array}$ & SSR/point \\
\hline all data kept & 445.005 & 65.4676 & 1.12251 & 0.0244513 \\
last data point & 334.291 & 77.1774 & 1.29804 & 0.0187503 \\
last 2 data points & 243.758 & 80.0209 & 1.5409 & 0.012798 \\
last 3 data points & 169.875 & 70.6113 & 1.908 & 0.00524038 \\
last 4 data points & 134.81 & 57.7649 & 2.21892 & 0.00345269
\end{tabular}




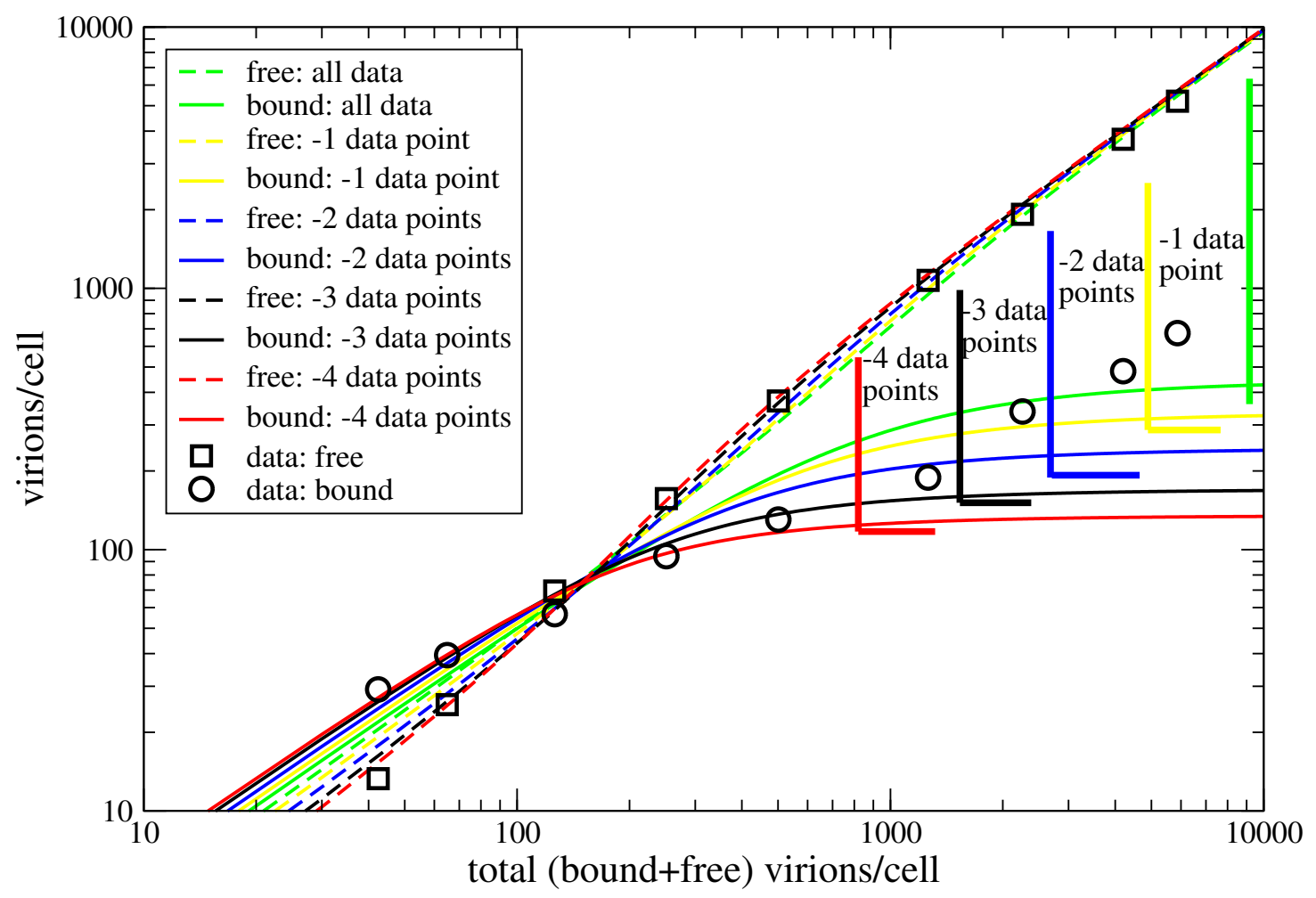

Figure 3.3: MM fits to bound and free virions vs total virions for multiple number of attachment sites. Nunes-Correia et al. infected MDCK cells at various MOIs in the presence of endocytosis inhibitors, where free and attached were enumerated at 20 minutes post infection. The simulation used all values, or disregarded up to the four highest MOIs [35].

Both MMs represent the free and attached virions at lower MOIs well, shown in Figure 3.4. We found that the MM fit, excluding the highest three MOI points, resulted in a simulation which catered well to all data points. We predict the number of general binding sites to be $\approx 170$. These parameter estimates were then used with fusion data. 


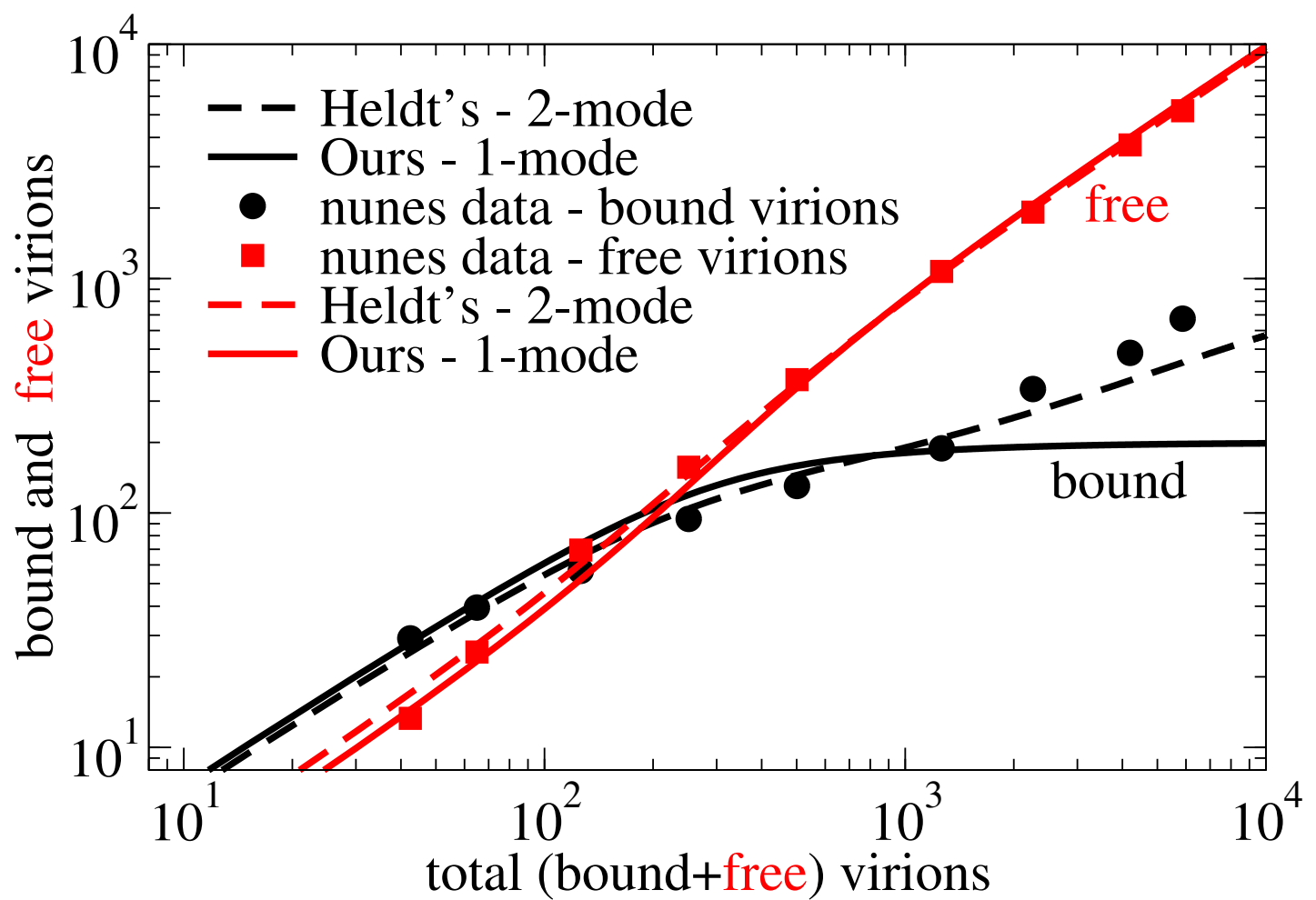

Figure 3.4: Comparison of Heldt et al.'s MM and our MM fit to free and attached virions. Although Heldt et al.'s MM has high and low affinity attachment terms and ours has one general entry term, both MMs do comparatively well at fitting the data. Our MM parameters are catered to lower MOIs since the highest 3 pairings of free and attached virions excluded during fitting of NunesCorreia et al. data.

Heldt et al.'s MM is able to fit the shape of the data at higher bound values, resulting a MM that estimates that attachment increases as MOI increases, however as stated, these very high MOIs are unlikely to be used in experiments or even to occur naturally. As such, we optimized the MM to the lower MOI data points, as seen in Figure 3.3. We estimated that there were 169.875 sites available for binding, with an attachment rate of 70.6113 sites $^{-1} \cdot \mathrm{h}^{-1}$ and an equilibrium constant of 1.908 site $^{-1}$ by excluding the last 3 data points, those being greater than an MOI of 2000 [35]. 


\subsection{Kinetics of virion entry and fusion}

We have shown that 2-mode attachment can be reduced in Heldt et al.'s MM and we progressed to examine endocytosis and release of vRNP segments into the cytoplasm. The following equations are from Heldt et al.'s MM:

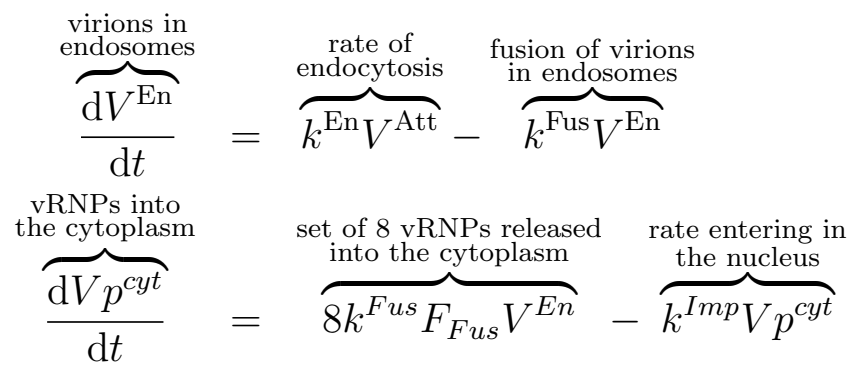

The following equations are from our MM:

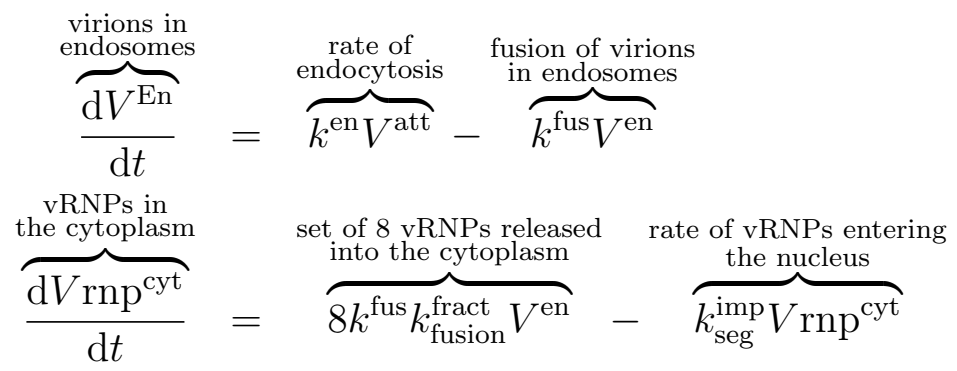

After attachment virions will endocytose at a rate of $k^{E n}$, represented by $V^{E n}$, where after they will fuse with early endosomes. Fusion occurs at a rate of $k^{\text {fusion, }}$, where the fusion competent virions, $k_{\text {fus }}^{\text {fract }}$, release eight negative sense viral strands from the endosome into the cytoplasm, $V \mathrm{rnp}^{\mathrm{cyt}}$. From here, the segments are imported into the nucleus at a rate $k_{\text {seg }}^{\mathrm{imp}}$, and are denoted as $V^{\text {neg }}$, as seen in Figure 2.1. No changes were made to the MM representation at this point.

We then examined if the endocytosis term needed to be explicitly written, or if the transition between attachment and viral proteins in the cytoplasm and nucleus 
could otherwise be represented as:

$$
\begin{aligned}
\frac{\mathrm{d} V^{\text {att }}}{\mathrm{d} t} & =k^{\text {att }}\left(1-V^{\text {att }} / N^{\text {sites }}\right) V^{\text {ex }}-k^{\text {det }} V^{\text {att }}-k^{\text {fus }} V^{\text {att }}-c V^{\text {Att }} \\
\frac{\mathrm{d} V \mathrm{rnp}^{\text {cyt }}}{\mathrm{d} t} & =8 k^{\text {fus }} k^{\text {fus }} V^{\text {att }}-k_{\text {seg }}^{\text {imp }} V \mathrm{rnp}^{\text {cyt }}
\end{aligned}
$$

i.e. omitting the $V^{\text {En }}$ term that appears in Heldt et al.'s MM between $V^{\text {att }}$ and $V \mathrm{rnp}^{\mathrm{cyt}}$.

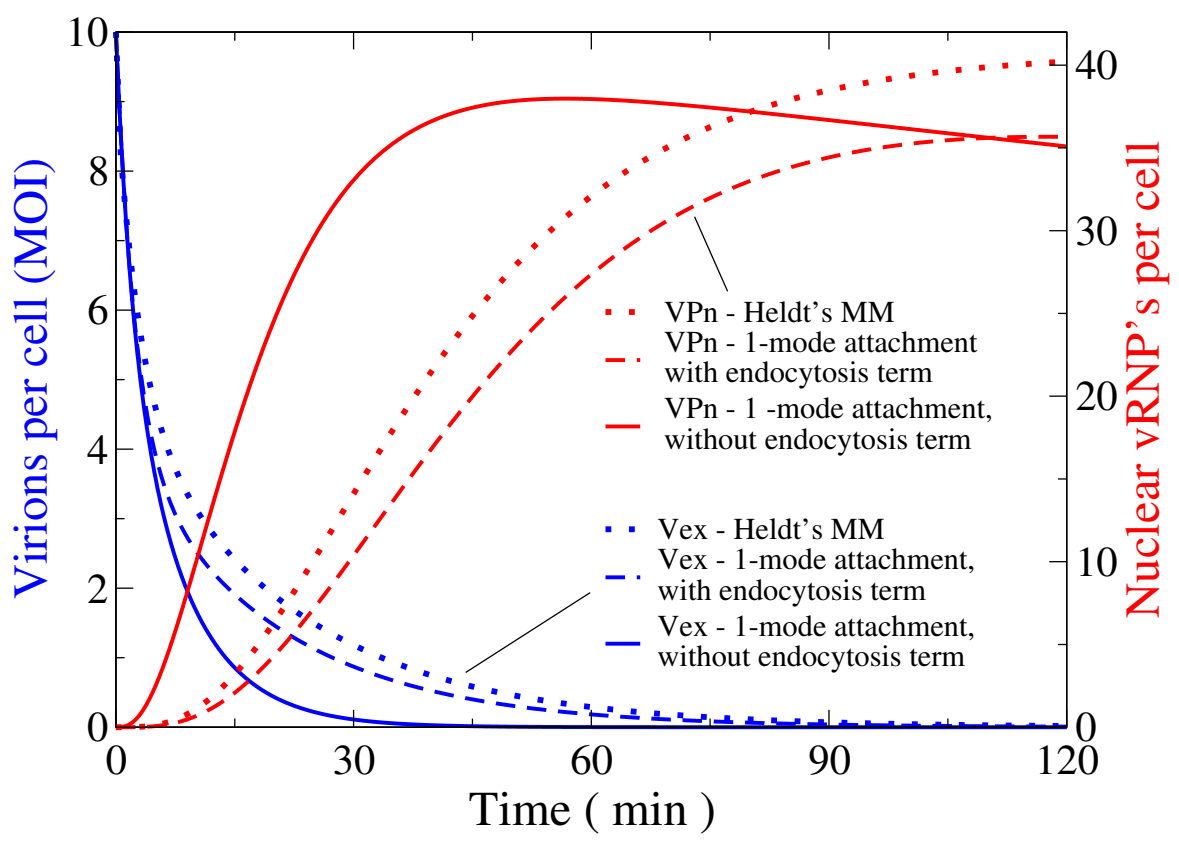

Figure 3.5: Comparison of virion entry from Heldt et al.'s and our MM. Our MM (with only one attachment term - dashed) reaches lower nuclear vRNP segment amounts than Heldt et al.'s MM (2 attachment terms - dots), however, both reach their maximum between 60 to 120 minutes post infection. Removal of the endocytosis term (in the 1-mode attachment term - solid) results in an earlier peak of nuclear vRNPs, approximately 45 minutes post infection. Infection was carried out at an MOI of 10 , resulting in 80 possible segments however, only $\approx 40$ will enter the nucleus as $51 \%$ get degraded prior. Both 1-mode attachment MMs were tested with the number of binding sites adjusted to 200 . 
As seen in Figure 3.5, the removal of the endocytosis term changes infection kinetics and would require adjustment of parameters. For the purposes of our MM, we keep the endocytosis term, and then turn to virion endosomal fusion events.

The number of virions fusing with endosomes was studied by Stegmann et al. at an MOI of 100. The first fusion events occur within 5 min after adsorption, with most fusion competent virions escaping late endosomes within 80 min. However, half of the adsorbed virions fail to fuse and are degraded in lysosomes [27]. In our MM the total number of fused virions can be obtained by integrating $\mathrm{k}^{\text {fus }} \mathrm{V}^{\mathrm{en}}$ over time which agrees well with experimental fusion data shown in Figure 3.6.

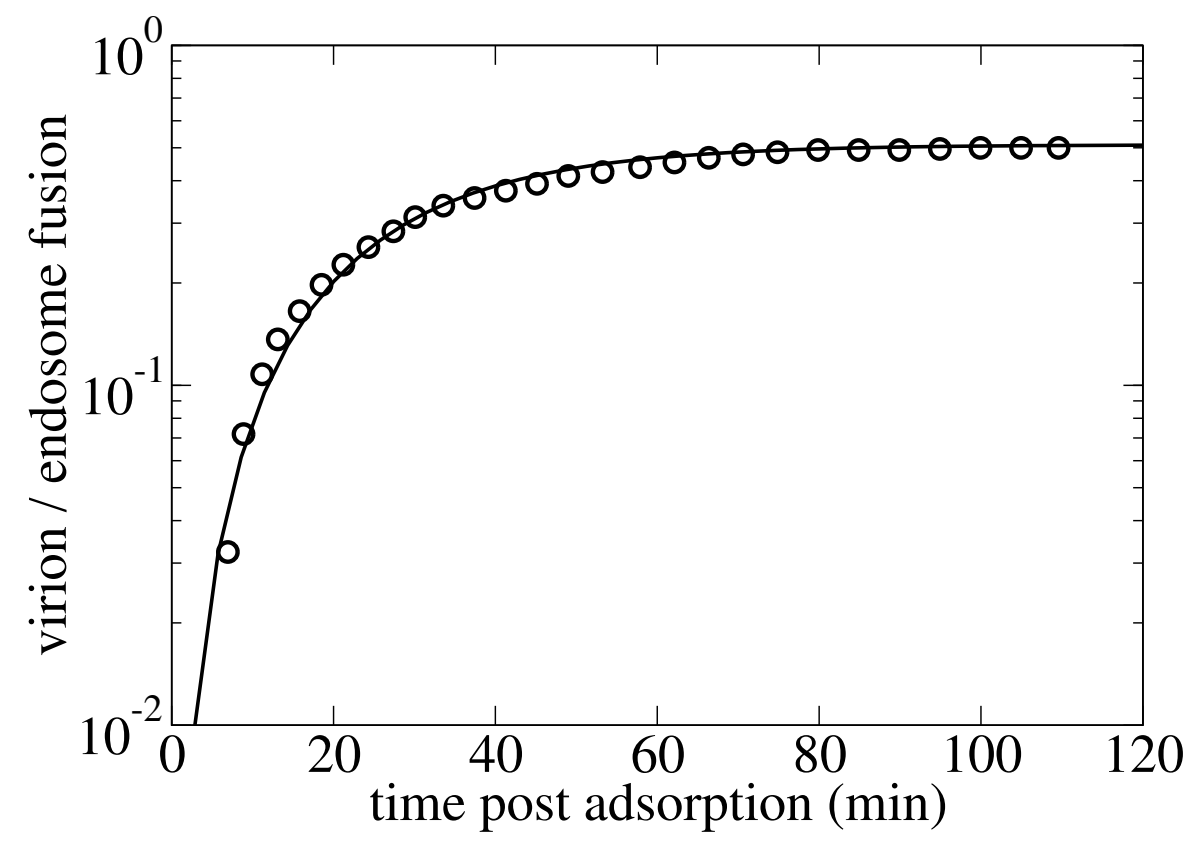

Figure 3.6: The percentage of virions fused out of total number of cellassociated virions. In this experiment Stegmann et al. infected MDCK cells with R-18 labelled IAV (strain NIB26) to measure fusion to endosomes. Cells were incubated for 1 hour at $0^{\circ} \mathrm{C}$, and washed so that unattached R-18 was removed, then viewed under florescence microscopy with continuous stirring where florescence is proportional to the fusion. Approximately half of the adsorbed virions fail to fuse and are degraded in lysosomes [27]. 
We determined the endocytosis rate $\left(k^{\mathrm{en}}\right)$ to be $7.24 \mathrm{~h}^{-1}$ which, is the same value for both high and low affinity attachment. The rate is within the range expected from Nunes-Corria et al. with the fusion rate being $3.7 \mathrm{~h}^{-1}\left(k^{\text {fus }}\right)$. The fraction of fusion competent virions $\left(k_{\text {fusion }}^{\text {fract }}\right)$ was set by experimental data from Stegmann et al. , where $51 \%$ of virions were found to be capable of fusing. The parameters determined from fitting of viral fusion data were used to compare infection kinetics of endocytosed and nuclear virions of our MM against Heldt et al.'s MM. As Figure 3.7 shows, 1-mode attachment and entry can properly represent infection kinetics.

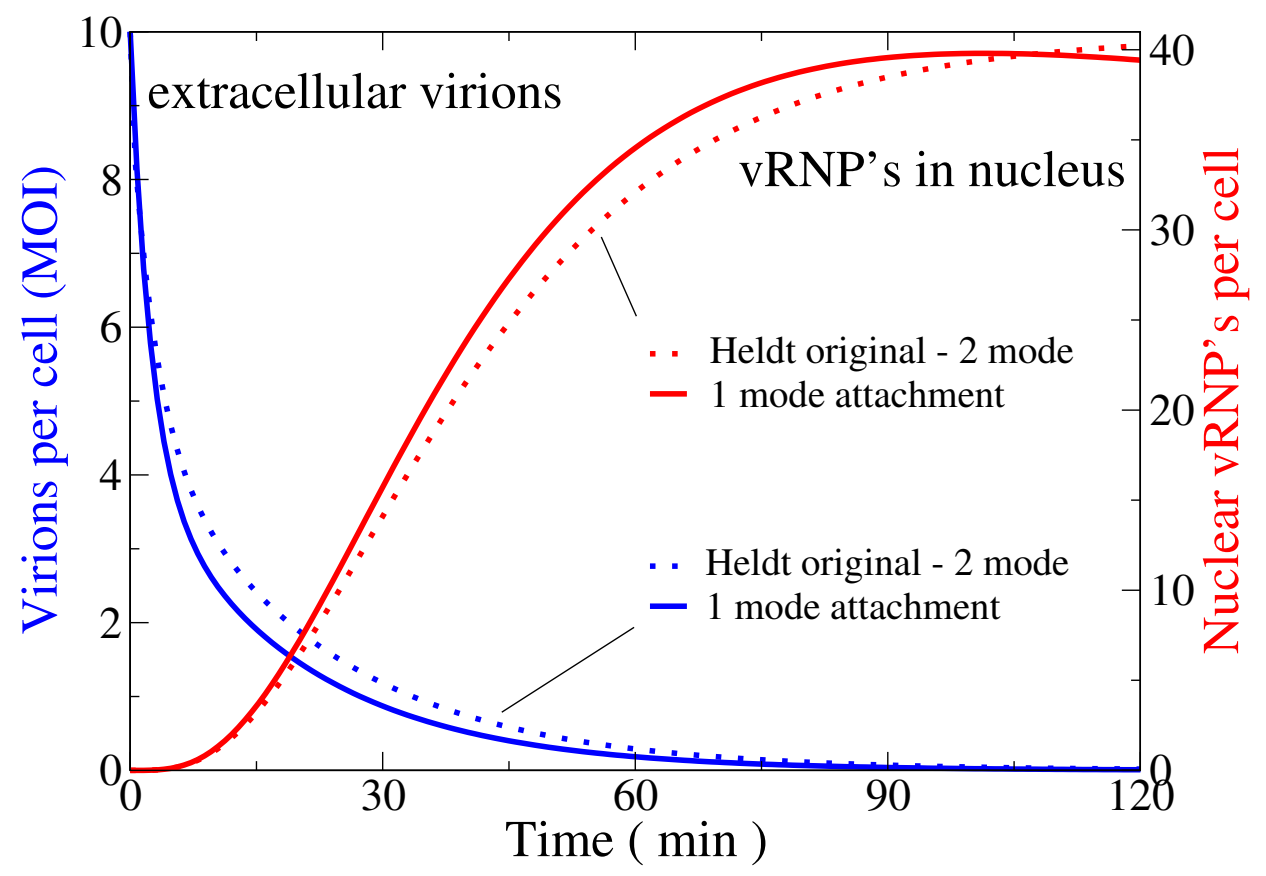

Figure 3.7: Rate of endocytosis and nuclear import. The MM was initiated with 10 virions in the extracellular medium (blue), and their appearance in the nucleus as vRNPs (red). Infection kinetics can be captured reasonably well when modelling virus entry solely through one mode of attachment [9]. 


\subsection{Kinetics of replication from fusion to progeny release}

The largest area of variable and parameter reduction by our MM, compared to that of Heldt et al., occurs in the steps between the import of vRNP into the nucleus to the assembly of progeny virions. In agreement with virological knowledge, cRNA, which requires stabilization by proteins, is required to produce progeny vRNA. Heldt et al. account for the production of stabilized cRNA through the incremental appropriation of proteins required. Produced $c R N A, \mathrm{R}^{C}$, is first bound by viral polymerase $\left(R_{R d R p}^{C}\right)$, then NP proteins $(C p)$. This stabilized cRNA, is used as a template in the synthesis of vRNA, $R^{V}$. The vRNA is stabilized by viral polymerase $\left(R_{R d R p}^{V}\right)$, then NP proteins to produce progeny $\mathrm{vRNP}\left(V p^{n u c}\right)$.

Heldt et al.'s MM from vRNP to cRNA to progeny vRNP:

$$
\begin{aligned}
& \overbrace{\frac{\mathrm{d} V p^{n u c}}{\mathrm{~d} t}}^{\text {nuclear VRNP }}=\overbrace{k^{I m p} V p^{c y t}}^{\begin{array}{c}
\text { rate entering in } \\
\text { the nucleus }
\end{array}}+\overbrace{k_{N P}^{\text {Bind } P_{N P} R_{R d R p}^{V}}}^{\begin{array}{c}
\text { vRNA-plymerase-NP complex } \\
=\text { vRNP }
\end{array}}-\overbrace{k_{M 1}^{\text {Bind } P_{M 1} V p^{n u c}}+\overbrace{k_{R n p}^{D e g} V p^{n u c}}^{\text {vRNP-M1 complex }}}^{\text {degradation }} \\
& \overbrace{\frac{\mathrm{d} R^{C}}{\mathrm{~d} t}}^{\text {cRNA }}=\overbrace{k_{C}^{S y n} V p^{\text {nuc }}}^{\text {cRNA synthesis }}-\overbrace{k_{R d R p}^{\text {Bind } P_{R d R p} R^{C}}}^{\begin{array}{c}
\text { cRNP formation } \\
R_{R d R p}^{C}
\end{array}}-\overbrace{k_{R}^{D e g} R^{C}}^{\begin{array}{c}
\text { degraded by } \\
\text { nucleases }
\end{array}}
\end{aligned}
$$

viral polymerase \& cRNA complexes

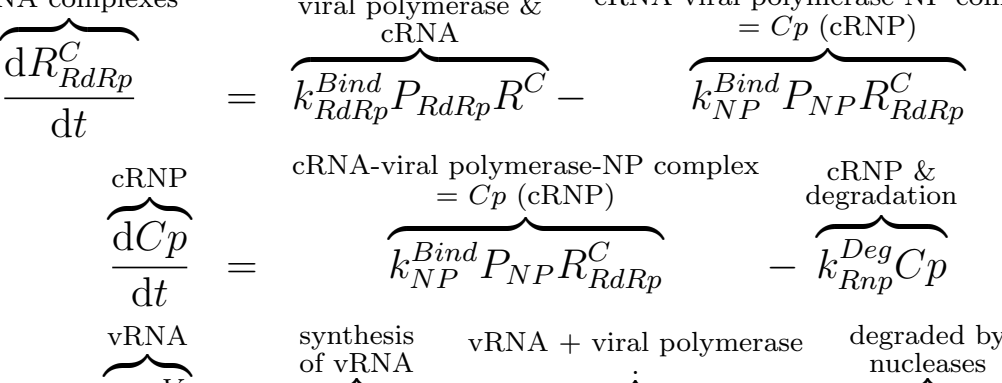

$$
\begin{aligned}
& \overbrace{\frac{\mathrm{d} R^{V}}{\mathrm{~d} t}}=\overbrace{k_{V}^{S y n} C p}^{\text {of vRNA }}-\overbrace{k_{R d R p}^{\text {Bind } P_{R d R p} R^{V}}}^{\text {vin }}-\overbrace{k_{R}^{\text {Deg }} R^{V}}^{\text {nucleases }}
\end{aligned}
$$

viral polymerase \& vRNA complex

$$
\overbrace{\frac{\mathrm{d} R_{R d R p}^{V}}{\mathrm{~d} t}}^{\text {RRA + viral polymerase }}=\overbrace{k_{R d R p}^{B i n d} P_{R d R p} R^{V}}^{\text {vRNA-viral polymerase-NP complex }}-\overbrace{k_{N P}^{B i n d} P_{N P} R_{R d R p}^{V}}^{\text {vRRNP }}-\overbrace{k_{R R d R p}^{D e g} R_{R d R p}^{V}}^{\text {Rvrr degradation }}
$$


We model the synthesis of cRNA $\left(V^{\text {cRNA }}\right)$ and the subsequent stabilized form of cRNA ( $\left.V^{\text {stab-cRNA }}\right)$ i.e. cRNA with NP proteins and viral polymerase. The equivalent process mathematically modelled by Heldt et al. individually accounts for the sequential addition rates of viral polymerase and NP to produce stabilized cRNA. We do not consider those intermediate steps where RNA will have bound a viral polymerase but not NP. By considering only successful complexes, we can reduce the number of equations in the MM representing replication as follows:

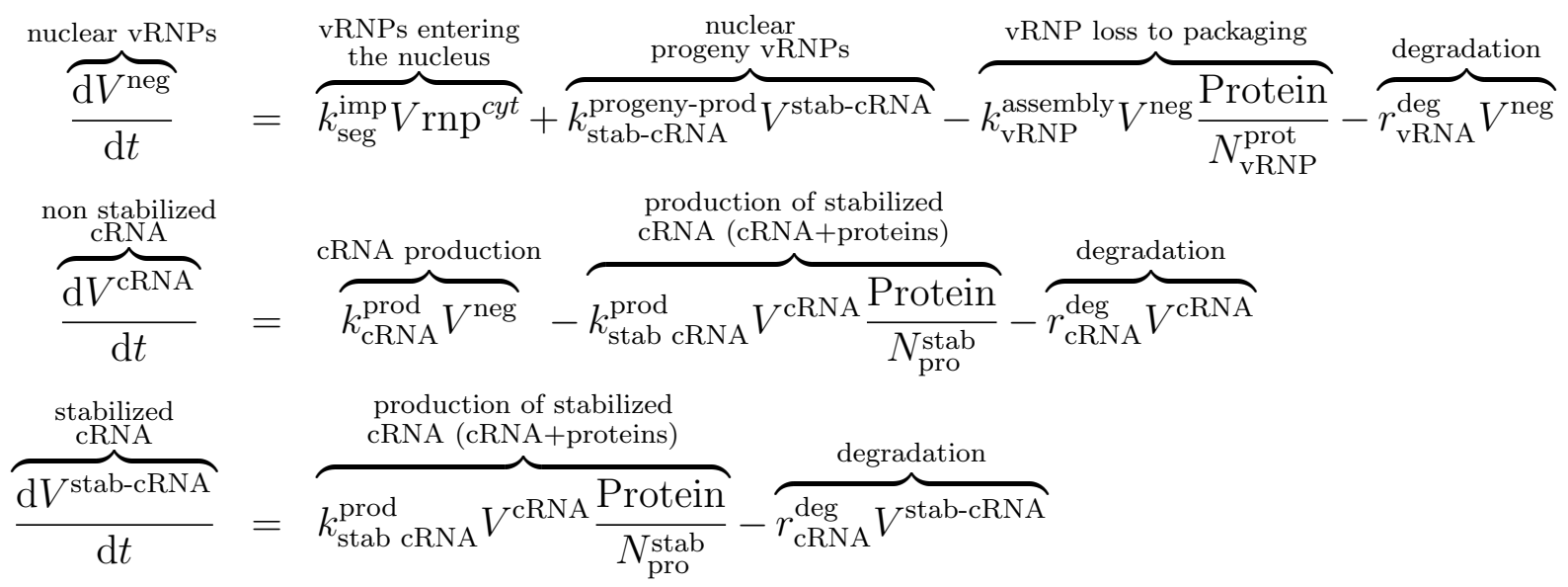

After endosomal escape and the release of 8 segments into the cytoplasm $\left(\mathrm{VP}^{\mathrm{cyt}}\right)$, the segments are imported into the nucleus (Vneg). The vRNP segments produce mRNA, which in turn produces proteins, that are either transported to the cell membrane, which become the coat of the progeny virion, or are imported back into the nucleus to stabilize cRNA and vRNPs.

Kawakami et al.'s experimental data, specifically the amount of mRNA, cRNA and vRNA, was used to fit mRNA, cRNA and vRNA variables in our MM. In the MM the amount of mRNA within a cell is represented by $V^{\mathrm{mRNA}}$ and $V^{\mathrm{cRNA}}$ and $V^{\text {stab-cRNA }}$ represent the total cRNA. vRNA experimental values are represented mathematically as the sum of $V^{\text {ex }}, V^{\text {att }}, V^{\text {en }}, V^{\text {neg }}, \operatorname{Vrnp}{ }^{\text {nucleus }}$ and $V_{\mathrm{ex}}^{\text {bud }}$.

For production of cRNA, there are currently two theories: switch and stabilization. The switching hypothesis concludes that after a virus has produced sufficient amount of mRNA it will task switch into making cRNA. However, recently in 
support of the stabilization theory, it has been shown that when a cell expresses required protein prior to infection, cRNA is detectable at early time points. Suggesting that cRNA is always made, but requires free viral polymerase and NP to be present to bind the nascent transcript to stabilize it [16]. Our MM requires proteins to be imported back into the nucleus to stabilize cRNA, $V^{\text {cRNA }}$, which would otherwise get degraded quickly by cellular pathways. In order to produce stabilized $\mathrm{cRNA}, V^{\text {stab-cRNA }}$, which can produce progeny vRNA, our MM requires sufficient viral proteins.

cRNA gets produced at a rate of $k_{\mathrm{cRNA}}^{\mathrm{prod}}$ and gets degraded at a rate of $r_{\mathrm{cRNA}}^{\mathrm{deg}}$, while the rate of production of one stabilized cRNA is $k_{\mathrm{cRNA}}^{\mathrm{stab}}$, which requires 80 proteins, and degrades at a rate of $r_{\text {stab-cRNA }}^{\text {deg }}$ The approximate number of proteins required to stabilize cRNA was determined by adding the three proteins required for formation of viral polymerase and the number of NP proteins on an average length vRNA segment. One NP is required per 24 nucleotides on a 1700 nucleotide long segment, representing an average length vRNA segment. Without sufficient protein production, stabilized cRNA would not be produced, which agrees with data from Vreede et al., who studied the stabilization hypothesis. They found that cRNA production was impeded when protein synthesis was inhibited, but was produced when cells were transfected with the required proteins prior to infection [16].

Proteins are an integral part of IAV replication, required for stabilization of both cRNA and vRNA, as well as incorporation into the nucleocapsids as transmembrane proteins. The production of mRNA segments $R_{i}^{M}$ is calculated based on the length $L_{i}$ of each viral segment, at synthesis rate, $k_{M}^{S y n}$, where $i$ is the segment type. Heldt et al.'s MM accounts for the production of different mRNA segments and viral proteins, while ours generalizes mRNA and protein production. 
While Heldt et al.'s MM represents production of mRNA using the following 8 equations:

$$
\begin{aligned}
\frac{\mathrm{d} R_{P B 1}^{M}}{\mathrm{~d} t} & =\frac{k_{M}^{S y n}}{L_{P B 1}} \frac{V p^{n u c}}{8}-k_{M}^{D e g} R_{P B 1}^{M} \\
\frac{\mathrm{d} R_{P B 2}^{M}}{\mathrm{~d} t} & =\frac{k_{M}^{S y n}}{L_{P B 2}} \frac{V p^{n u c}}{8}-k_{M}^{D e g} R_{P B 2}^{M} \\
\frac{\mathrm{d} R_{P A}^{M}}{\mathrm{~d} t} & =\frac{k_{M}^{S y n}}{L_{P A}} \frac{V p^{n u c}}{8}-k_{M}^{D e g} R_{P A}^{M} \\
\frac{\mathrm{d} R_{H A}^{M}}{\mathrm{~d} t} & =\frac{k_{M}^{S y n}}{L_{H A}} \frac{V p^{n u c}}{8}-k_{M}^{D e g} R_{H A}^{M} \\
\frac{\mathrm{d} R_{N A}^{M}}{\mathrm{~d} t} & =\frac{k_{M}^{S y n}}{L_{N A}} \frac{V p^{n u c}}{8}-k_{M}^{D e g} R_{N A}^{M} \\
\frac{\mathrm{d} R_{N P}^{M}}{\mathrm{~d} t} & =\frac{k_{M}^{S y n}}{L_{N P}} \frac{V p^{n u c}}{8}-k_{M}^{D e g} R_{N P}^{M} \\
\frac{\mathrm{d} R_{M 1}^{M}}{\mathrm{~d} t} & =\frac{k_{M}^{S y n}}{L_{M 1}} \frac{V p^{n u c}}{8}-k_{M}^{D e g} R_{M 1}^{M} \\
\frac{\mathrm{d} R_{N S 1}^{M}}{\mathrm{~d} t} & =\frac{k_{M}^{S y n}}{L_{N S 1}} \frac{V p^{n u c}}{8}-k_{M}^{D e g} R_{N S 1}^{M}
\end{aligned}
$$


and the following 10 equations to represent protein production:

$$
\begin{aligned}
\frac{\mathrm{d} P_{P B 1}}{\mathrm{~d} t} & =\frac{k_{P}^{S y n}}{D_{R i b}} R_{2}^{M}-k^{R d R p} P_{p B 1} P_{p B 2} P_{p A} \\
\frac{\mathrm{d} P_{P B 2}}{\mathrm{~d} t} & =\frac{k_{P}^{S y n}}{D_{R i b}} R_{1}^{M}-k^{R d R p} P_{p B 1} P_{p B 2} P_{p A} \\
\frac{\mathrm{d} P_{P A}}{\mathrm{~d} t} & =\frac{k_{P}^{S y n}}{D_{R i b}} R_{3}^{M}-k^{R d R p} P_{p B 1} P_{p B 2} P_{p A} \\
\frac{\mathrm{d} P_{R d R p}}{\mathrm{~d} t} & =k^{R d R p} P_{p B 1} P_{p B 2} P_{p A}-k_{R d R p}^{\text {Bind }} P_{R d R p}\left(R^{V}+R^{C}\right)-\left(N_{P_{R d R p}}-8\right) r^{R e l} \\
\frac{\mathrm{d} P_{N P}}{\mathrm{~d} t} & =\frac{k_{P}^{S y n}}{D_{R i b}} R_{5}^{M}-\frac{L_{V}}{N_{N P}^{N u c}} k_{N P}^{\text {Bind }} P_{N P}\left(R_{R d R p}^{V}+R_{R d R p}^{C}\right)-\left(N_{P_{N P}}-8 \frac{L_{V}}{N_{N P}^{N u c}}\right) r^{R e l} \\
\frac{\mathrm{d} P_{M 1}}{\mathrm{~d} t} & =\frac{k_{P}^{S y n}}{D_{R i b}}\left(1-F_{S p 17}\right) R_{7}^{M}-\frac{L_{V}}{N_{N P}^{N u c}} k_{M 1}^{B i n d} P_{M 1} V p^{N u c}-\left(N_{P_{M 1}}-8 \frac{L_{V}}{N_{M 1}^{N u c}}\right) r^{R e l} \\
\frac{\mathrm{d} P_{N E P}}{\mathrm{~d} t}= & \frac{k_{P}^{S y n}}{D_{R i b}} F_{S p 18} R_{8}^{M}-\frac{L_{V}}{N_{N E P}^{N u c}} k^{E x p} P_{N E P} V p_{M 1}^{n u c}-\left(N_{P_{N E P}}-8 \frac{L_{V}}{N_{N E P}^{N u c}}\right) r^{R e l} \\
\frac{\mathrm{d} P_{H A}}{\mathrm{~d} t}= & \frac{k_{P}^{S y n}}{D_{R i b}} R_{4}^{M}-N_{P_{H A}} r^{R e l} \\
\frac{\mathrm{d} P_{N A}}{\mathrm{~d} t}= & \frac{k_{P}^{S y n}}{D_{R i b}} R_{6}^{M}-N_{P_{N A}} r^{R e l} \\
\frac{\mathrm{d} P_{M 2}}{\mathrm{~d} t}= & \frac{k_{P}^{S y n}}{D_{R i b}} F_{S p 17} R_{7}^{M}-N_{P_{M 2}} r^{R e l}
\end{aligned}
$$

our MM simplifies this process to just 3 equations:

$$
\begin{aligned}
\frac{\mathrm{d} V^{\mathrm{mRNA}}}{\mathrm{d} t} & =r_{\mathrm{mRNA}}^{\mathrm{prod}} V^{\mathrm{neg}}-r_{\mathrm{mRNA}}^{\mathrm{deg}} V^{\mathrm{mRNA}} \\
\frac{\mathrm{dProtein}}{\mathrm{d} t} & =N_{\mathrm{rib}} k_{\text {pro }}^{\mathrm{synth}} \frac{5.5}{8} V^{\mathrm{mRNA}}-k_{\mathrm{cRNA}}^{\mathrm{stab}} V^{\mathrm{cRNA}} \text { Protein }-k_{\mathrm{vRNP}}^{\text {assembly }} V^{\text {neg }} \text { Protein } \\
\frac{\mathrm{dProtein}{ }^{\mathrm{Memb}}}{\mathrm{d} t} & =N_{\mathrm{rib}} k_{\mathrm{pro}}^{\mathrm{synth}} \frac{2.5}{8} V^{\mathrm{mRNA}}-k_{\mathrm{pro}}^{\mathrm{memb}} \operatorname{Protein}^{\text {Memb }}
\end{aligned}
$$

This simplification is reasonable as we only consider average segment lengths. Heldt et al. model the production of each segment, taking into consideration the length of the segment and how many ribosomes can fit on an mRNA segment to transcribe protein. This approach is required in cases where unique segments are required for specific events i.e. NP binding to cRNA, which may be rate limiting 
when made in low quantities. We see however, in Figure 3.8 that proteins in Heldt et al.'s MM are made in abundance that although they have different rates of production, they all become non-rate limiting early on. As such our simplification to the production rate of an average mRNA and protein segment can be sufficient.

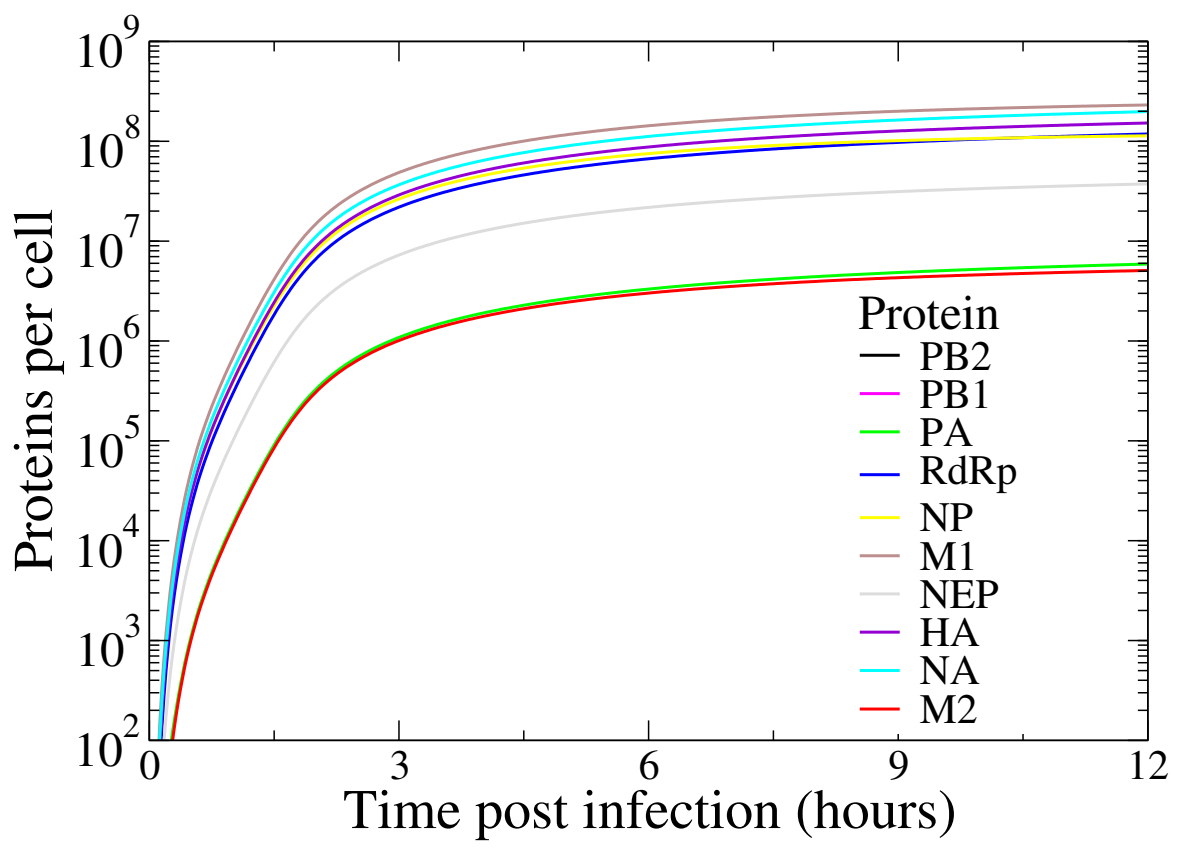

Figure 3.8: Protein expression based on Heldt et al.'s MM. Reproduction of Heldt et al.'s protein level simulations. Each segment is expressed in varying orders of magnitude (2 orders) but all display similar kinetics. Proteins are quickly in excess of amount required and do not limit replication.

The import of proteins into the nucleus stabilizes the cRNA, thus allowing production of progeny vRNA which, also requires protein for formation of vRNPs. Proteins affect the switch from transcription and translation to export and, although we utilize proteins in our MM we do not use literature data to constrain Protein and Protein ${ }^{\mathrm{Memb}}$. We calculate the number of ribosomes that can fit on one average length mRNA segment to be $10.625, N_{\text {rib }}$, however the production of proteins $k_{\text {pro }}^{\text {synth }}$, is not well identified. We consider that protein segment levels in our MM are significantly high (reaching a maximum of $2 \cdot 10^{8}$ proteins per segment) and are thus not a limiting factor. 
We do not consider the trafficking of the envelope proteins between the endoplasmic reticulum and the budding site. This multi-step pathway is normally used by the cell to identify misfolded proteins and proteins requiring post translational modification, as such we assume the cell culture used in experimentation is healthy. We assumed that the materials required for the virus to properly reproduce, such as ribosomes, are fully functional in a cell. We also assume that building blocks, such as amino acids and nucleotides, are sufficiently abundant that they don't hinder replication. The synthesis of the membrane proteins (M2, HA and NA) is done by the host ribosomes, and the amount of amino acids consumed in one cell by the infection was previously mathematically modelled by Sidorenko et al. Based on dry weight analysis, it is estimated that there are $3.1 \times 10^{10}$ free amino acids/cell $[48,49]$, a number they found to be sufficiently high that it does not present itself as a rate limiting factor for infection $[10,50]$.

Heldt et al. use 18 variables to represent mRNA synthesis and protein translation, which we represent in our MM by 3 variables. We represent all mRNAs $\left(V^{\mathrm{mRNA}}\right)$ and proteins (Protein and Protein $\left.{ }^{\mathrm{Memb}}\right)$ through average synthesis rates based on a 1700 nt long segment of vRNA. Heldt et al. account for each individual segment lengths during transcription and translation, and through examination of the mRNA and protein levels, we find that although shorter segments are produced in higher quantities, they all reach significantly high levels. Additionally we saw that the kinetics of proteins were sufficiently similar that we felt the MM could be simplified by having an average length segment, which we calculated to be 1700 nt long. This length is used to calculate how many NP proteins are used to stabilize one cRNA, with one NP circling 24 nt, plus PB1, PB2 and PA as the viral polymerase. We also calculate that 537.5 proteins are required to coat one vRNP prior to nuclear export [18]. 
Lastly we consider the packaging and release of progeny virions. Heldt et al.'s MM represent assembly and release using the following 3 equations:

vRNP-M1 complex

$$
\frac{\overbrace{\mathrm{d} V p_{M 1}^{n u c}}^{\text {in nucleus }}}{\mathrm{d} t}
$$

Cytoplasmic NEP-M1-vRNP complexes

$$
\frac{\overbrace{\mathrm{d} V p_{M 1}^{c y t}}^{\mathrm{d} t}}{\begin{array}{c}
\text { assembly } \\
\text { and release }
\end{array}}
$$

$$
=\overbrace{k_{M 1}^{\text {Bind } P_{M 1} V p^{n u c}}}^{\begin{array}{c}
\text { vRNP-M1 } \\
\text { complexes }
\end{array}}-\overbrace{k^{E x p} P_{N E P} V p_{M 1}^{n u c}}^{\begin{array}{c}
\text { vRNP-NP-M1-NEP complex being } \\
\text { exported from nucleus }
\end{array}}+\overbrace{k_{R n p}^{D e g} V p_{M 1}^{n u c}}^{\text {degradation }}
$$

$$
\begin{aligned}
\overbrace{\frac{\mathrm{d} V^{\text {Rel }}}{\mathrm{d} t}=} & r^{\text {Rel }}=k^{\text {Rel }} V p_{M 1}^{c y t} \prod \frac{P_{R d R p}}{P_{R d R p}+K_{V^{R e l}} N_{P_{R d R p}}} \frac{P_{H A}}{P_{H A}+K_{V \text { Rel }} N_{P_{H A}}} \\
& \frac{P_{N P}}{P_{N P}+K_{V R e l} N_{P_{N P}}} \frac{P_{N A}}{P_{N A}+K_{V R e l} N_{P_{N A}}} \frac{P_{M 1}}{P_{M 1}+K_{V^{R e l}} N_{P_{M 1}}} \\
& \frac{P_{M 2}}{P_{M 2}+K_{V \text { Rel }} N_{P_{M 2}}} \frac{P_{N E P}}{P_{N E P}+K_{V \text { Rel }} N_{P_{N E P}}}
\end{aligned}
$$

and our MM represents this process with 3 equations:

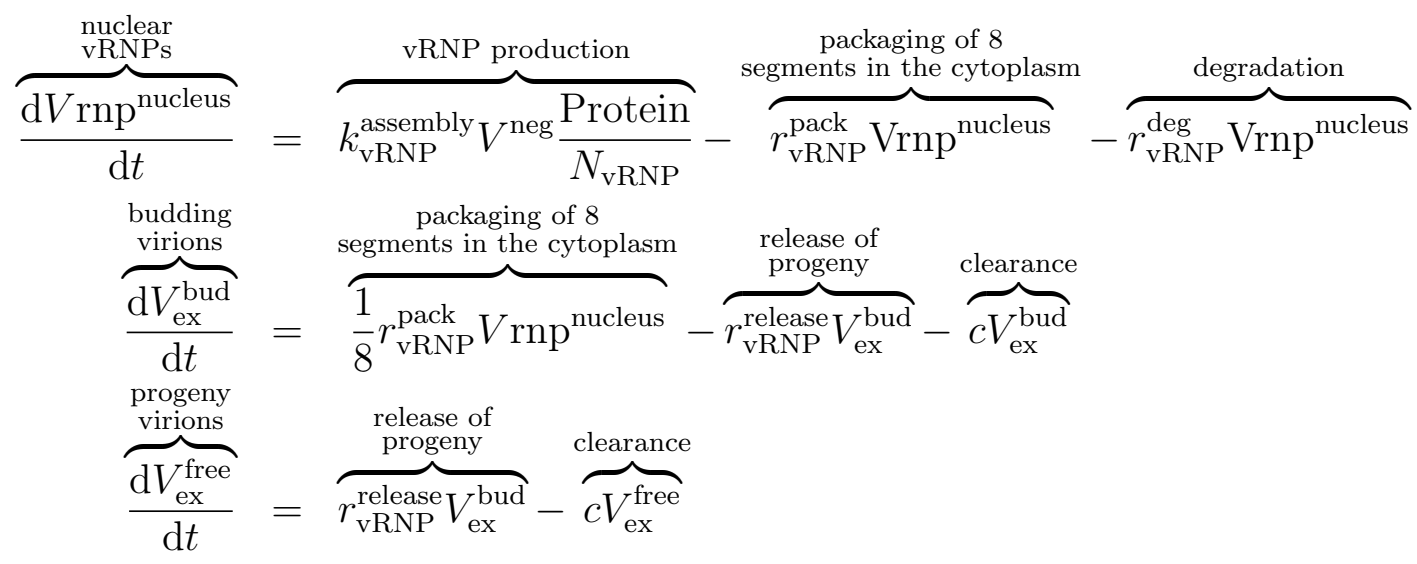

In Heldt et al.'s MM nuclear vRNPs $\left(V p_{M 1}^{n u c}\right)$ are exported to the cytoplasm $\left(V p_{M 1}^{c y t}\right.$, at a rate of $\left.k^{E x p}\right)$ where eight $v R N P s$ and proteins, are packaged and bud from the cell surface $\left(V^{R e l}\right)$. In the same fashion of production of cRNA 
and vRNA, individual proteins, such as M1 and NEP $\left(P_{M 1}, P_{N E P}\right)$ are added sequentially to vRNPs, at a rates of $k_{M 1}^{B i n d}$ and $k^{E x p}$ respectively. The rate of viral release, $r^{\text {Rel }}$ includes the virion assembly rate $r^{R e l}$. We proceed through this in an opposite manner; we represent the gaining of proteins in one step ( $V \mathrm{rnp}^{\text {nucleus }}$ by rate $k_{\mathrm{vRNP}}^{\text {assembly }}$ ), and include a term to represent budding, $V_{\mathrm{ex}}^{\text {bud }}$, with a rate of $r_{\mathrm{vRNP}}^{\text {pack }}$. Heldt et al.'s decay of $V p_{M 1}^{n u c}$ and $V p_{M 1}^{c y t}$ (by rate $k_{R n p}^{D e g}$ and $k_{R n p}^{D e g}$ respectively) is the equivalent to our $V \mathrm{rnp}^{\text {nucleus }}$ (at a rate of $k_{R n p}^{D e g}$ ). In Heldt et al.'s MM, the release of virions occurs when there is sufficient protein of each type per virion. We consider that protein availability is not a rate limiting step here, and we represent the release rate $\left(r_{\mathrm{vRNP}}^{\text {release }}\right)$ of budding virions, $V_{\mathrm{ex}}^{\text {bud }}$, to the extracellular medium, $V_{\mathrm{ex}}^{\text {free }}$.

We account for budding explicitly, as we cater to the incorporation of antivirals, in this case neuraminidase inhibitors, which functions by blocking release at the budding step. We also incorporate a clearance rate to both budding and free virions which represents physical degradation a virion particle or loss of infectivity.

Heldt et al. mathematically modelled the transition between protein synthesis and progeny viral genome well, taking into account the stabilization hypothesis for complementary RNA which requires certain viral proteins in the nucleus. Although this MM simulates experimental data, it does so though adjustment of parameters i.e. values are adjusted so that one portion of the infection can better represent a data set. In total there are six distinct sets of parameters, resulting in the adjustment of ten parameters, used for modelling. When one examines these changes model wide it can result in large differences in values attained for all monitored variables. 

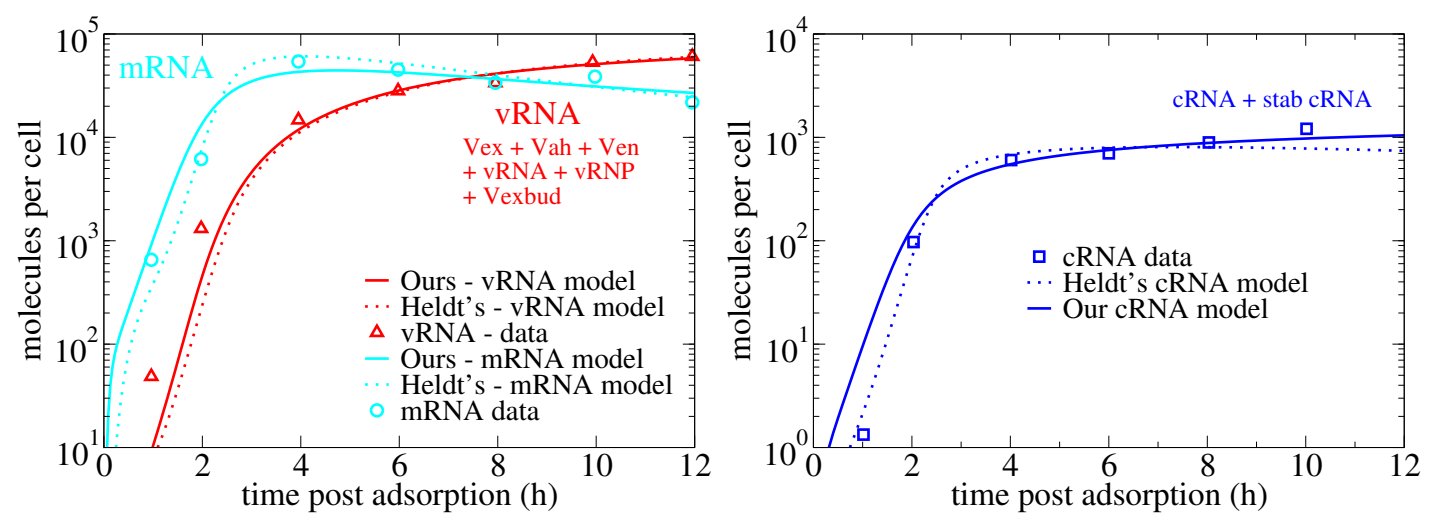

Figure 3.9: Comparing our MM and fitted parameters to experimental mRNA, vRNA and cRNA data. In this experiment MDCK cells were infected with IAV strain A/WSN/33 (H1N1) at an MOI of 10. Average numbers of RNA copies in an infected cell were determined by strand-specific real-time RT-PCR [Data digitized from Kawakami, 2011 and MM from Heldt et al., 2012] [9,36].

The values for the fitted parameters are reported in Table 2.6, with the data set used to determine those values denoted where applicable. Our MM has 13 variables and 25 parameters, a reduction of 19 variables and 23 parameters compared to Heldt et al.'s MM. Comparing our MM (initial infection to release) and fitted parameters to the experimental data yielded good results.

\subsection{Extracellular validation of the intracellular MM}

After validation of the intracellular kinetics we use the MM to simulate in silico 'experiments' and from these to make predictions about the kinetics of infection. However, can one cell represent a whole cell culture to bridge the divide? To do so, we must first make an assumption that one cell can represent a whole cell culture, which it can when all cells are infected at the same time. 
By infecting a cell culture with a high virus MOI, all cells will be infected at approximately the same time, meaning that they will all progress through infection mostly simultaneously, in a single cycle, as seen in Figure 3.10. The type of infection kinetics can then be compared to that of a single cell. Although one may assume that given an MOI of 1 infectious virion per cell, each cell would be infected by one virion. But it is likely that some cells will get, 2, 1, or no virions. This means that cells which were inoculated at the same time can theoretically be offset in the timing of their actual infection.

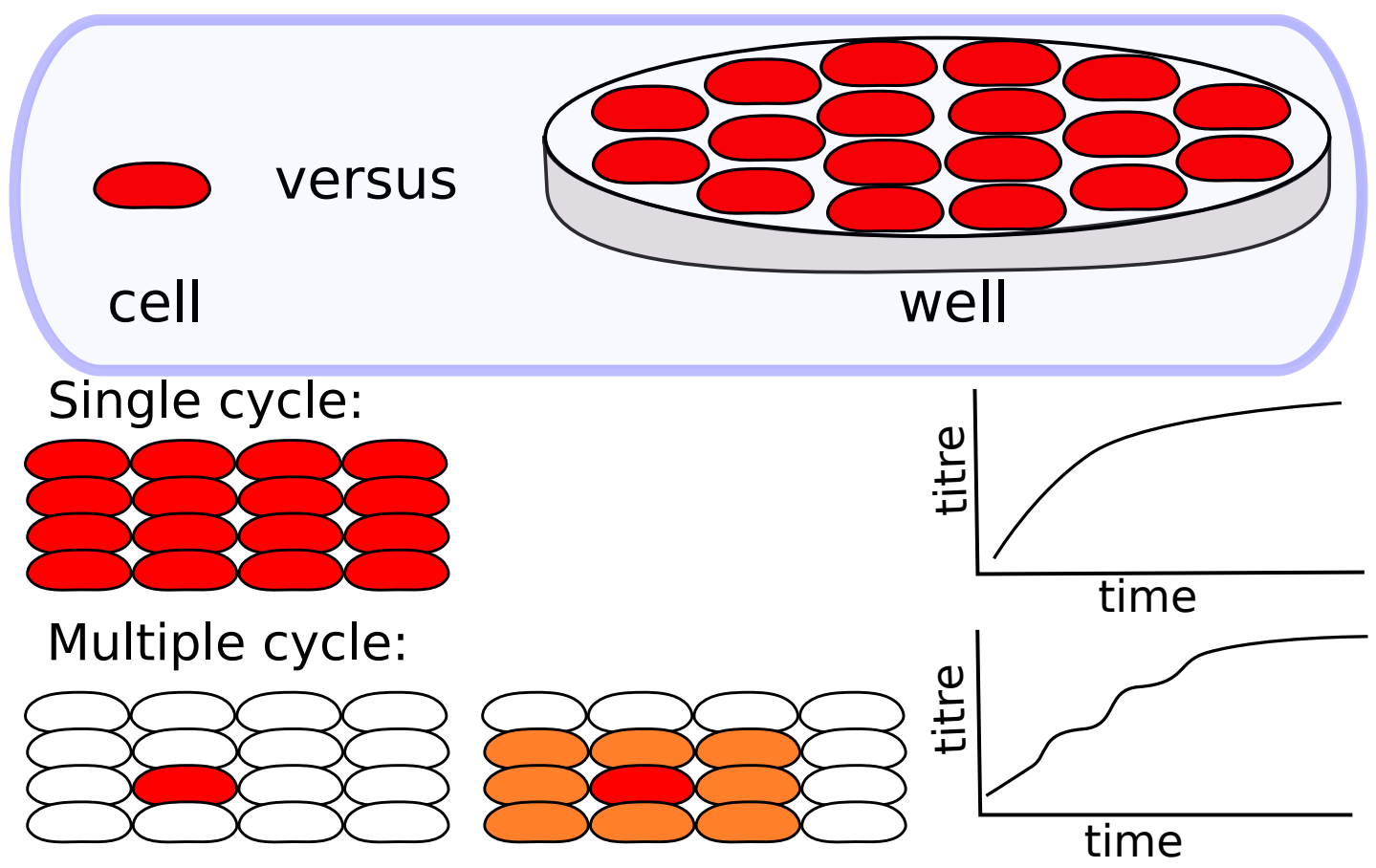

Figure 3.10: Single vs multiple cycle experiments. The behaviour of cell populations can be consistent to that of a single cell when infected at high enough MOIs that all cells would become infected at the same time. Low MOI infections have rounds of infection, placing cells at various stages of infection at any given time.

Our MM for viral replication in a single cell cannot be compared to viral kinetics from infections at low MOIs, as only a portion of cells get infected, and they would therefore go through rounds of infection asynchronously, as the infection spreads 
from cell to cell. We could only account for such low MOI infections if we utilized our MM in a multicellular model and tracked all infections and their neighbouring uninfected target cells. Previously, large MOI experiments were excluded from parameter extraction, however, here we state that high MOIs are required. To clarify this, the difference is the interpretation of what is considered 'large' or 'high'. In attachment experiments MOIs of > 2000 virions per cell were considered too large to be biologically relevant, however, for cell culture experiments considered by our MM, high MOIs are those $>4$ virions per cell. High MOI experiments result in synchronous cell infection, which allows us to compare the kinetics of cell cultures to that of a single cell.

Though our MM is intracellular, it must also capture extracellular kinetics $\left(\mathrm{V}^{\mathrm{ex}}\right)$, the experimentally observed growth kinetics of the extracellular virus concentration in the supernatant. We compare our MM against Heldt et al.'s MM in capturing an infection at an MOI of 4, using parameters determined though fitting. We find both MMs do well in predicting the viral titre time course observed experimentally, as shown in Figure 3.11. 


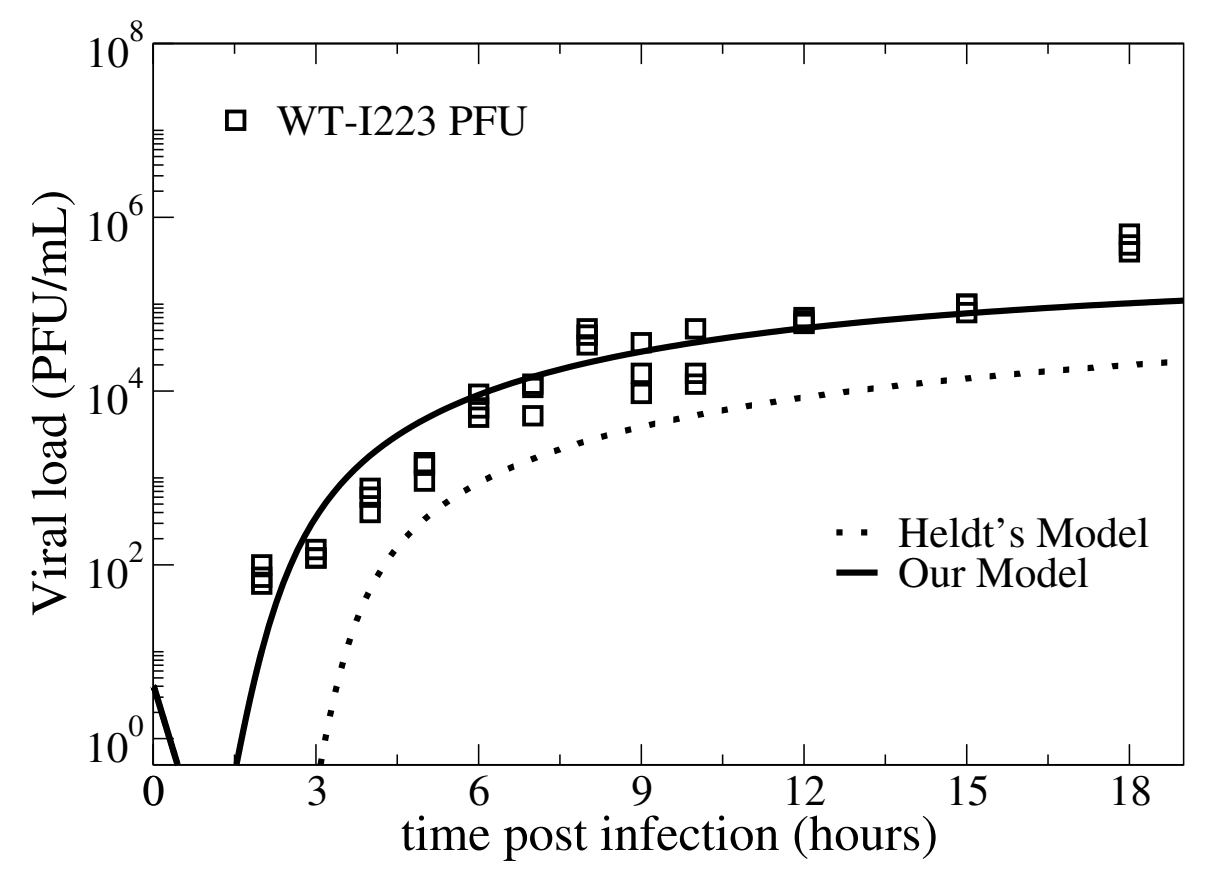

Figure 3.11: Comparing the two MMs to extracellular viral kinetics. Single-cycle IAV infection with H1N1pdm09 WT-I223 at an MOI of 4 was conducted on Madin-Darby canine kidney cells modified to have similar expression of $\alpha-2,6$ sialic acid receptors as those found on human upper respiratory epithelial cells. PFU experimental data points were collected in triplicate at each time point. We simulated Heldt et al.'s MM (dashed line) and our MM (solid line) under experimental conditions outlined in the publication (Data digitized from Paradis 2015, Heldt et al.'s MM re-simulated) [9,38].

We adjusted the infection in our MM to match the conditions of the experiments, and looked at the progeny virions produced in Figure 3.11. Our MM does well at predicting the progeny virions, and does so with 23 fewer parameters and 19 less variables than Heldt et al.'s MM. Although our MM does better in this instance, it does not imply that our MM is better. Each experiment can yield slightly different results due to the IAV strain used, experimental variability, and the averaging of cell behaviour from populations, such that one might argue that given a different experiment Heldt et al.'s MM would better match the data. 
Although Heldt et al.'s MM can accurately capture all kinetics of infection, it does so through adjustment of parameters to produce different figures. Included as an example is Figure 3.12, where parameters used by the MM to produce late infection kinetics are reincorporated for early events. Parameters used when mathematically modelling viral production cannot be used for recapitulating attachment of free virions. This adjustment of rates is done to match experimental data at different times in the infection process, i.e. not in cases such as setting $\mathrm{k}^{\mathrm{en}}$ to zero when endocytosis inhibitors were used in experimentation. Our newly developed concise MM for the intracellular kinetics of IAV infection in vitro could function with one set of 'best fit' parameters, reported in Table 2.6, can reproduce literature data, i.e. attachment, fusion, and RNA data, without requiring changes in the rates of different processes.

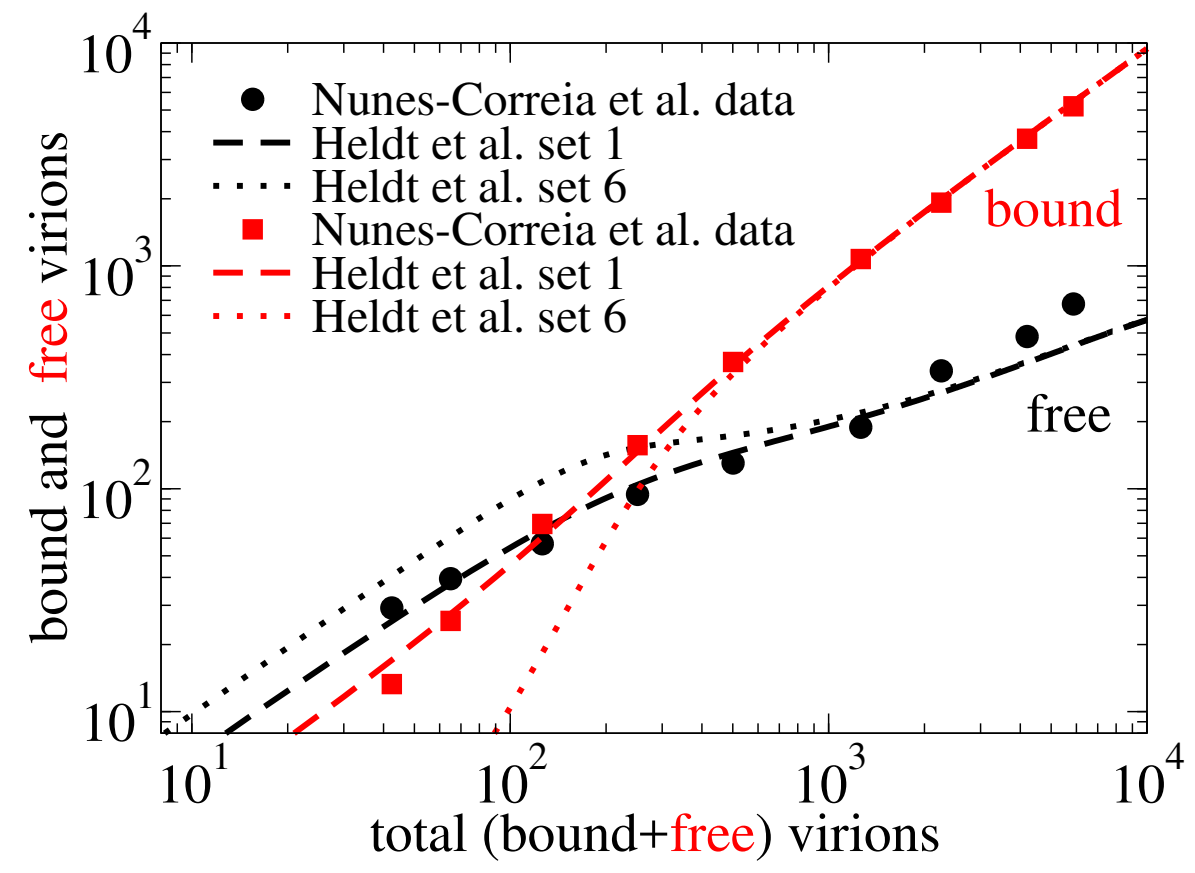

Figure 3.12: Bound and free virions estimated with Heldt et al.'s MM. We simulated Heldt et al.'s MM catered to viral release (set 6 - dotted line) and when used to determine attachment and detachment rates (set 1 - dashed line) to match experimental conditions by Nunes-Correia et al. (Data digitized from Nunes-Correia et al., Heldt et al.'s MM re-simulated) [9, 35]. 


\subsection{Using the MM to ask key virological ques- tions}

\subsubsection{Replication kinetics under super-infection}

After illustrating that our MM is sufficiently capable of modelling intra- and extracellular data, we use the MM to preform in silico experiments. These simulations allow us to estimate the kinetics of infection in vitro under conditions which may otherwise be under the limit of detection. Experimentation has a great deal of cell to cell variability, and often results are a product of an average cell behaviour, which can be misleading if all cells are not synchronously infected.

If two genetically different viruses infect the same cell at the same time, both can replicate, and this could result in reassortment. We explore the effect of timing and inoculum concentration on superinfection. Antigenetic shift due to superinfection results from the re-assortment of viral RNA. However, IAV employs methods of blocking of superinfection, namely by expression of the surface protein neuraminidase which cuts off sialic acid receptors that are required for attachment of virions. This leads to the question of how long after initial infection can a cell be superinfected, thus resulting in the re-assortment of genetic information? This question has been loosely addressed using time of addition curves, i.e. experiments which use offset secondary infections to affect initial infections. Early on, secondary infections greatly affect infection kinetics but, after some time additional virions entering the system have no effect on the initial replication $[51,52]$.

Virions are imperfect, and can bind cellular debris or can enter a cell but unsuccessfully produce an infection [9]. To further our MM, we intend on incorporating the effect of a naturally occurring phenomenon known as defective interfering particles (DIPs), since they play a large role in infection kinetics. DIPs are virus particles that possess an incomplete genome, and often cannot complete infection by themselves, but are not easily quantifiable. When DIPs co-infect a cell with a productively infectious virion, the DIPs out-compete productively infectious virions by hijacking their means of replication i.e. stealing their polymerase. The 
presence of DIPs in a sample will be observed as a decrease in infectious viral yield, because DIPs reduce the number of productively infectious virions produced via competition $[52,53]$.

What happens to the virus production rate when a cell is singly vs multiply infected is affected by the amount of virions infecting a cell. A representative cell was singly infected at time zero in our MM with one virion, rinsed at 30 minutes post infection, and its virus production was compared to that for superinfected cells with varying inoculum.

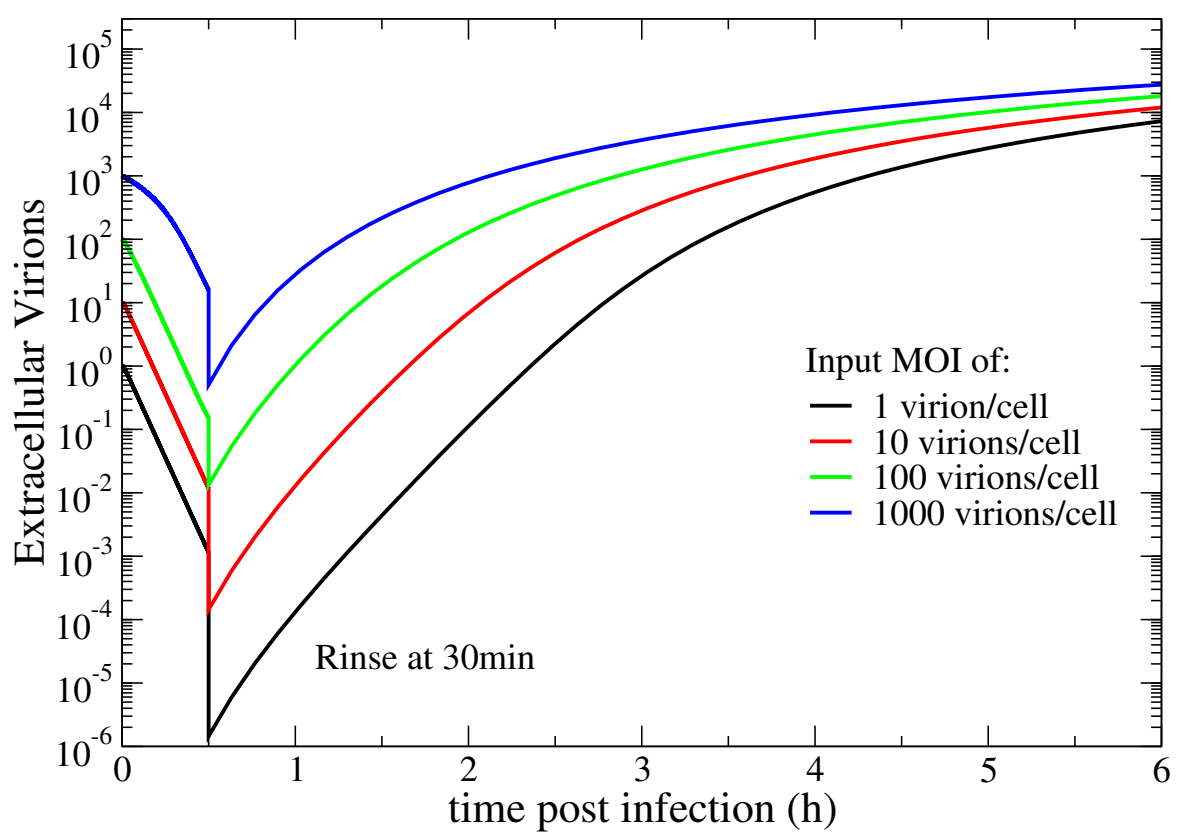

Figure 3.13: Predicting viral titres for increasing input MOIs. Cells were infected at time zero with inoculum of various concentrations which were allowed to adsorb for 30 minutes and were then rinsed.

Given the MM parameters determined, our MM predicts that increased input MOI results in shorter eclipse phase, i.e. hastened virus production, as seen in Figure 3.13. 


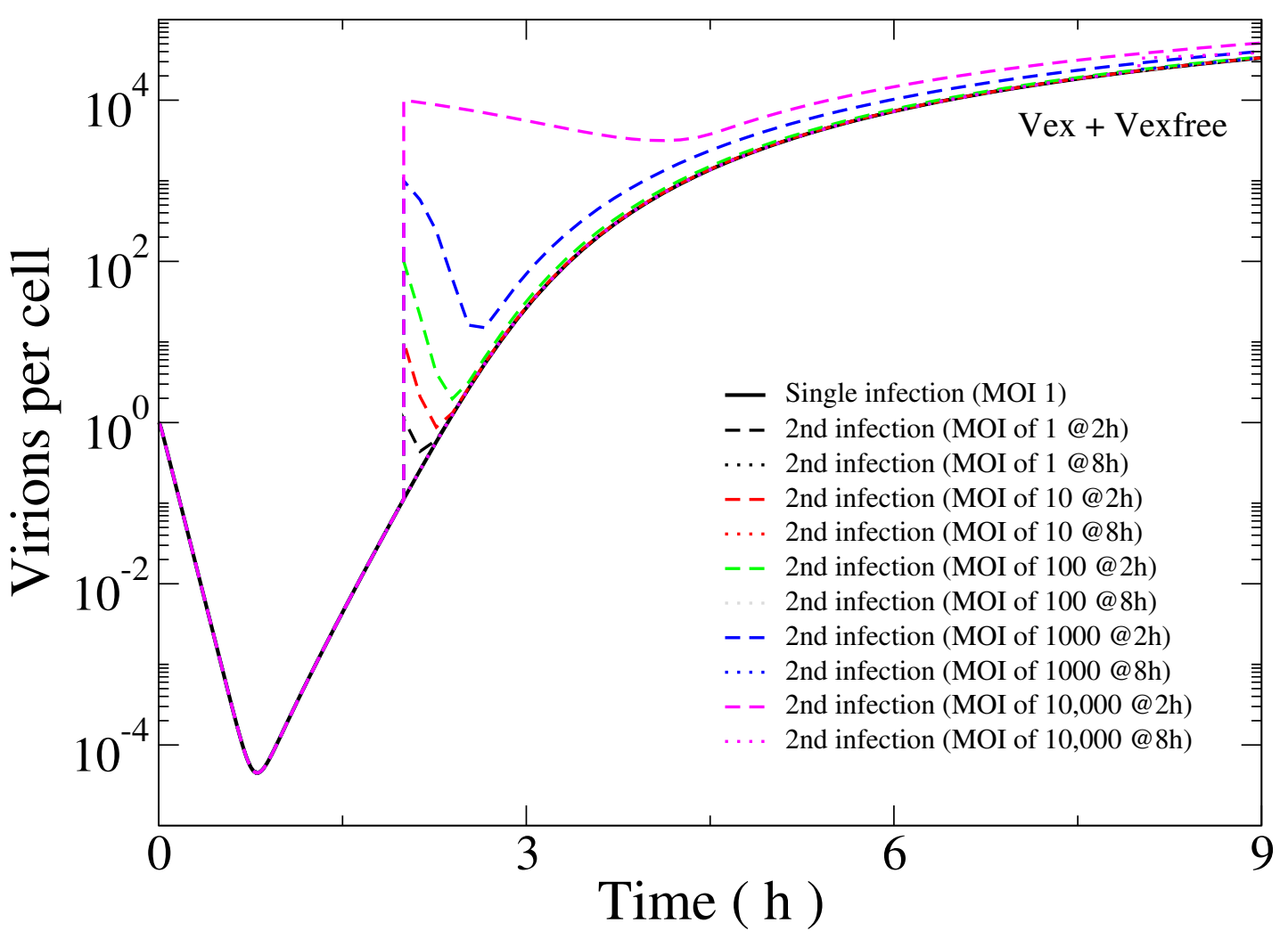

Figure 3.14: Effect of superinfection timing on production. The MM simulated the addition of 1 virion at time 0 , with secondary infections of various MOIs at either $2 \mathrm{~h}$ or $8 \mathrm{~h}$ post initial infection. At $2 \mathrm{~h}$, the addition of 100 virions has little to no effect on viral release, while the addition of 1000 virions hastens the release slightly. There is no discernible effect when secondary inoculums are administered at $8 \mathrm{~h}$, unless a significantly large inoculum of 10,000 virions/cell is added.

The kinetics of infection are not largely affected by the addition of virions at later times points, indicating that secondary infections can be obscured when additions are smaller than the progeny virions produced. The effect of superinfection at early time points, shown in Figure 3.15, have a greater impact the earlier the secondary inoculum is administered. 


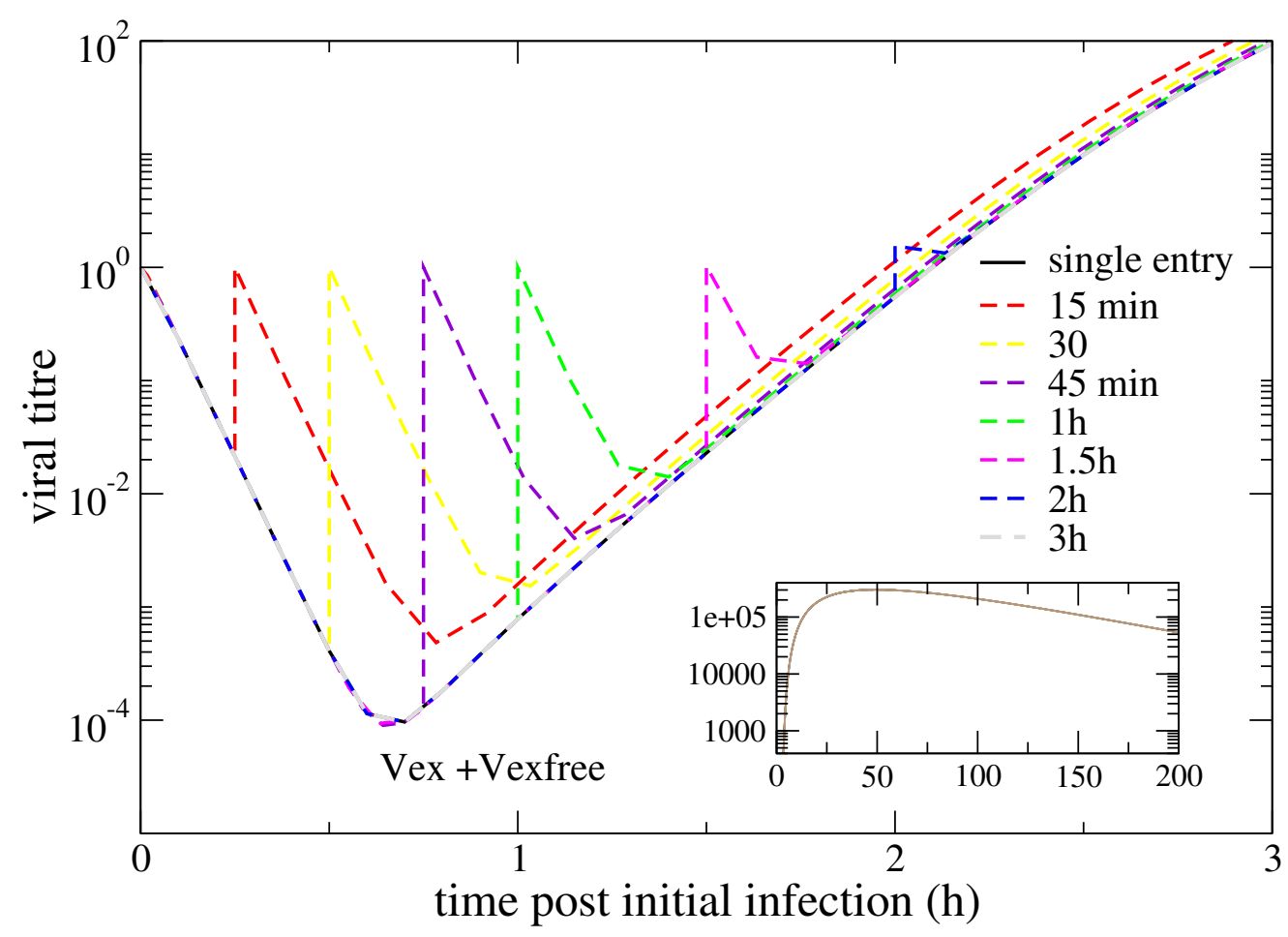

Figure 3.15: Effect of early superinfection timing on production. At time zero, a cell is inoculated with one virion, where after secondary infections by another single virion after which a secondary inoculum consisting of a single virion is added at various times up to $3 \mathrm{~h}$ post initial inoculum.

The addition of 1 virion followed by a second single virion after various hours produces a hastened production, where larger effects are seen with earlier administration of secondary inocula. However, as the Figure 3.15 inset illustrates, the overall kinetics are not significantly affected. As illustrated also in Figure 3.14, secondary inoculations which are on the same order of magnitude as the viral release at the same time can be masked by the latter. For this reason, we used secondary inocula of 100 and 1000 virions/cell, administered at earlier time points, shown in Figure 3.16. It is interesting to note that Figure 3.15 seems to illustrate that by $1 \mathrm{~h}$ secondary infections have no impact on infection kinetics. However, by simulating the addition of larger inocula, we can see that an impact still occurs at $2 \mathrm{~h}$ (Figure $3.16)$. 


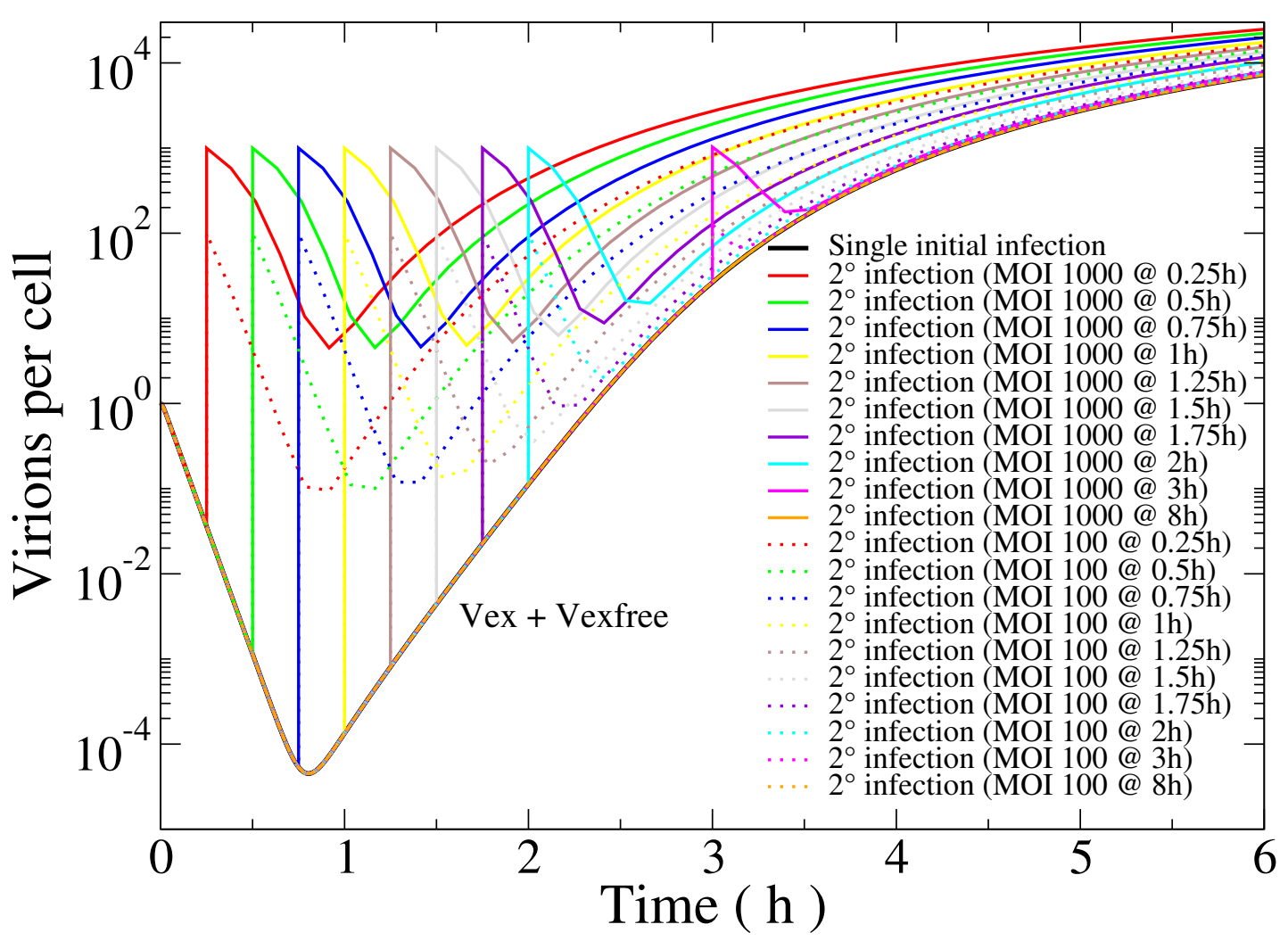

Figure 3.16: Effect of superinfection dose on production. Addition of 1 initial virion followed by subsequent infections at various times up to 8 hours at MOIs of 100 and 1000 virion/cell.

Testing large secondary infections can allow us to see how long superinfection can have an impact on infection. Figure 3.16 illustrates the addition of 1 initial virion at time $0 \mathrm{~h}$, followed by subsequent infections at various times at MOIs of $100 \& 1000$. When added within $2-3 \mathrm{~h}$ post-infection, the second inoculum shortens the eclipse phase, but does not impact the viral yield. It has no apparent impact if added $3 \mathrm{~h}$ or more after the initial infection. 


\subsubsection{Efficacy of different antiviral targets}

When taken within the first 2 days of infection, antiviral treatment can lessen the symptoms associated with the infection, and for those with underlying conditions, can be the difference between a mild illness or a hospitalization. There are several classes of antiviral inhibitors: fusion inhibitors, which block the M2 protein channel, viral polymerase inhibitors, which cause mutations during replication, and neuraminidase inhibitors, which compete for occupation of the active site of NA. Our MM must be able to represent the mode of action of all of these key antiviral targets, shown in Figure 3.17. 


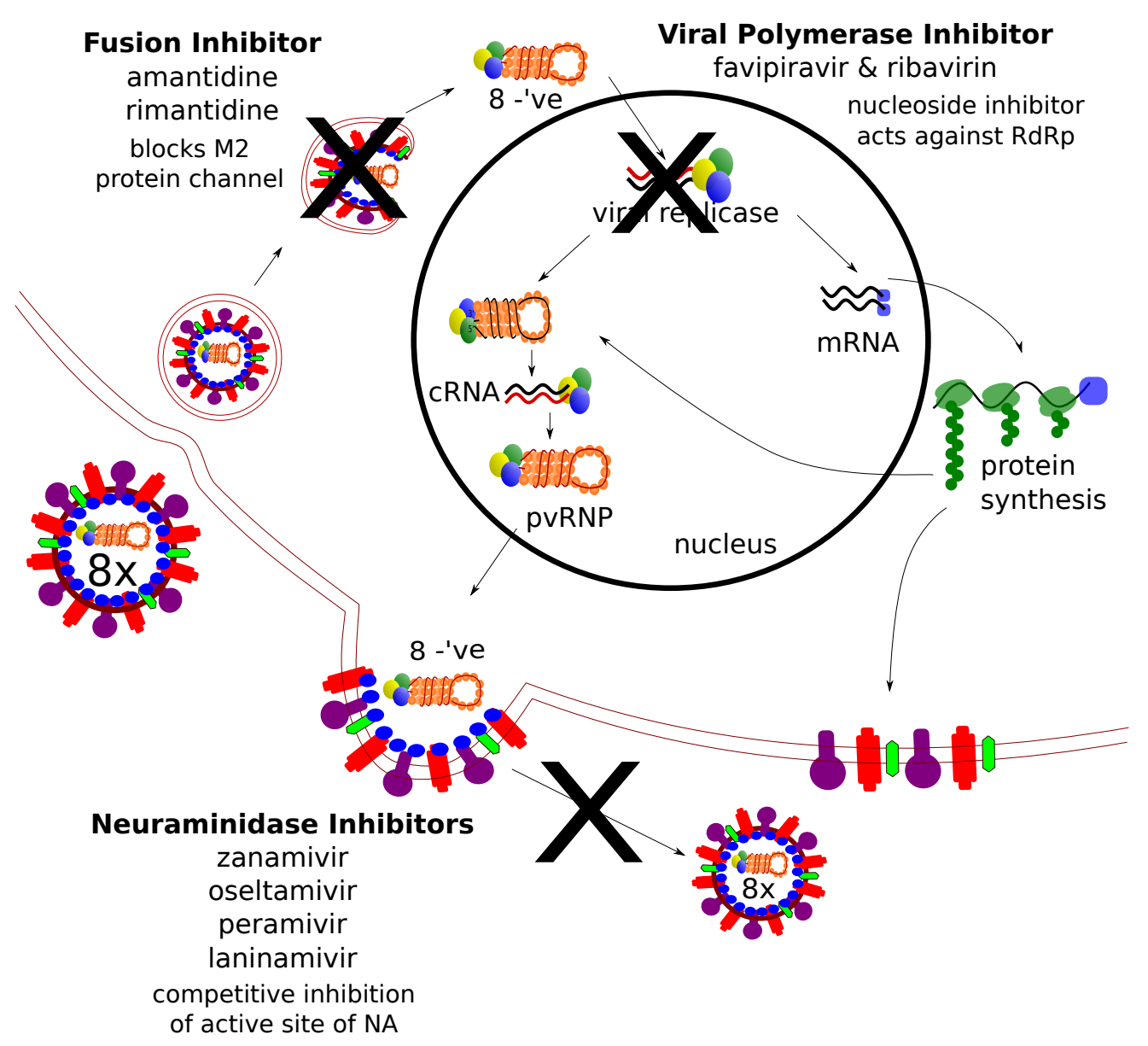

Figure 3.17: Antiviral mode of action. The MM was built such that common antivirals could be incorporated easily, such as inhibitors of fusion, viral polymerase inhibitors, and neuraminidase inhibitors.

As we incorporate antivirals into our MM, they will be represented by the addition of $(1-\varepsilon)$ term, where $\varepsilon$ is the efficacy of the drug, which increases with increased drug concentration. Fusion inhibitors are incorporated where a virion will uncoat after acidification in the endosome. Viral polymerase inhibitors are incorporated where RNA dependant RNA polymerase acts, such as in the production of progeny $\mathrm{V}^{\text {neg }}$. Neuraminidase inhibitors mimic the natural substrate of NA and fit into the active site preventing the budding of progeny from the host cell to the extracellular medium. As such, they are included into the MM where they block the release of budded virions. 
Mathematically, we can represent the action of these different inhibitors in our MM as:

$$
\begin{aligned}
& \frac{\mathrm{d} V^{\mathrm{Ex}}}{\mathrm{d} t}=k_{\mathrm{det}} V^{\mathrm{Att}}-k_{\mathrm{hi}}^{\mathrm{Att}}\left(1-V^{\mathrm{att}} / N^{\mathrm{sites}}\right) V^{\mathrm{Ex}}-\mathrm{c} V^{\mathrm{Ex}} \\
& \frac{\mathrm{d} V^{\mathrm{Att}}}{\mathrm{d} t}=k^{\mathrm{Att}}\left(1-V^{\mathrm{att}} / N^{\mathrm{sites}}\right) V^{\mathrm{ex}}-k_{\mathrm{det}} V^{\mathrm{att}}-k^{\mathrm{en}} V^{\mathrm{att}}-\mathrm{c} V^{\mathrm{att}} \\
& \frac{\mathrm{d} V^{\mathrm{En}}}{\mathrm{d} t}=k^{\mathrm{en}} V^{\mathrm{att}}-k^{\mathrm{fus}} V^{\mathrm{en}} \\
& \frac{\mathrm{d} V \mathrm{rnp}^{\mathrm{cyt}}}{\mathrm{d} t}=8 k^{\mathrm{fus}}(1-\varepsilon) k_{\text {fus }}^{\mathrm{fract}} V^{\mathrm{en}}-k_{\mathrm{seg}}^{\mathrm{imp}} V \mathrm{rnp}^{\mathrm{cyt}} \\
& \frac{\mathrm{d} V^{\text {neg }}}{\mathrm{d} t}=k_{\mathrm{seg}}^{\mathrm{imp}} V \operatorname{rnp}^{\text {cyt }}+(1-\varepsilon) k_{\text {stab-cRNA }}^{\text {progeny-prod }} V^{\text {stab-cRNA }} \\
& -k_{\mathrm{vRNP}}^{\text {assembly }} V^{\text {neg }} \frac{\text { Protein }}{N_{\mathrm{vRNP}}^{\text {prot }}}-r_{\mathrm{vRNA}}^{\mathrm{deg}} V^{\text {neg }} \\
& \frac{\mathrm{d} V^{\mathrm{mRNA}}}{\mathrm{d} t}=(1-\varepsilon) r_{\mathrm{mRNA}}^{\mathrm{prod}} V^{\mathrm{neg}}-r_{\mathrm{mRNA}}^{\mathrm{deg}} V^{\mathrm{mRNA}} \\
& \frac{\mathrm{dProtein}}{\mathrm{d} t}=N_{\text {rib }} k_{\text {pro }}^{\text {synth }} \frac{5.5}{8} V^{\mathrm{mRNA}}-k_{\text {cRNA }}^{\text {stab }} V^{\text {cRNA }} \text { Protein }-k_{\text {vRNP }}^{\text {assembly }} V^{\text {neg }} \text { Protein } \\
& \frac{\text { dProtein }^{\text {Memb }}}{\mathrm{d} t}=N_{\text {rib }} k_{\text {pro }}^{\text {synth }} \frac{2.5}{8} V^{\text {mRNA }}-k_{\text {pro }}^{\text {memb }} \operatorname{Protein~}^{\text {Memb }} \\
& \frac{\mathrm{d} V^{\mathrm{cRNA}}}{\mathrm{d} t}=(1-\varepsilon) k_{\mathrm{cRNA}}^{\mathrm{prod}} V^{\mathrm{neg}}-k_{\mathrm{cRNA}}^{\mathrm{stab}} V^{\mathrm{cRNA}} \frac{\text { Protein }}{N_{\mathrm{pro}}^{\text {stab }}}-r_{\mathrm{cRNA}}^{\mathrm{deg}} V^{\mathrm{cRNA}} \\
& \frac{\mathrm{d} V^{\text {stab-cRNA }}}{\mathrm{d} t}=k_{\mathrm{cRNA}}^{\text {stab }} V^{\text {cRNA }} \frac{\text { Protein }}{N_{\text {pro }}^{\text {stab }}}-r_{\text {stab-cRNA }}^{\text {deg }} V^{\text {stab-cRNA }} \\
& \frac{\mathrm{d} V \mathrm{rnp}^{\text {nucleus }}}{\mathrm{d} t}=k_{\mathrm{vRNP}}^{\text {assembly }} V^{\text {neg }} \frac{\text { Protein }}{N_{\mathrm{vRNP}}^{\text {prot }}}-r_{\mathrm{vRNP}}^{\text {pack }} \operatorname{Vrnp}^{\text {nucleus }}-r_{\mathrm{vRNP}}^{\text {deg } \operatorname{Vrnp}}{ }^{\text {nucleus }} \\
& \frac{\mathrm{d} V_{\mathrm{ex}}^{\text {bud }}}{\mathrm{d} t}=\frac{1}{8} r_{\mathrm{vRNP}}^{\text {pack }} V \operatorname{rnp}{ }^{\text {nucleus }}-(1-\varepsilon) r_{\mathrm{vRNP}}^{\text {release }} V_{\mathrm{ex}}^{\text {bud }}-\mathrm{c} V_{\mathrm{ex}}^{\text {bud }} \\
& \frac{\mathrm{d} V_{\mathrm{ex}}^{\text {free }}}{\mathrm{d} t}=(1-\varepsilon) r_{\mathrm{vRNP}}^{\text {release }} V_{\mathrm{ex}}^{\mathrm{bud}}-\mathrm{c} V_{\mathrm{ex}}^{\text {free }}
\end{aligned}
$$

Fusion inhibitors affect the release of virions into the cytoplasm $V \mathrm{rnp}^{\text {cyt }}$, which we capture in our MM as reducing the amount fusion events, $\left(k_{\text {fus }}^{\text {fract }}\right)$. Viral polymerase inhibitors affect viral polymerase, which is required for several integral processes; mRNA $\left(V^{\mathrm{mRNA}}\right)$, cRNA $\left(V^{\mathrm{cRNA}}\right)$ and vRNA $\left(V^{\text {neg }}\right)$ production, which we capture in our MM as reducing the amount fusion events, effectively reducing $k_{\mathrm{cRNA}}^{\mathrm{prod}}, r_{\mathrm{mRNA}}^{\mathrm{prod}}$ and $k_{\text {stab-cRNA }}^{\text {progeny-prod }}$ respectively. Lastly, the action of neuraminidase inhibitors is cap- 
tured in our MM as reducing the rate of release of virions $\left(r_{\text {vRNP }}^{\text {release }} V\right)$, causing a reduction in the production rate, progeny virions $\left(V_{\mathrm{ex}}^{\text {free }}\right)$.
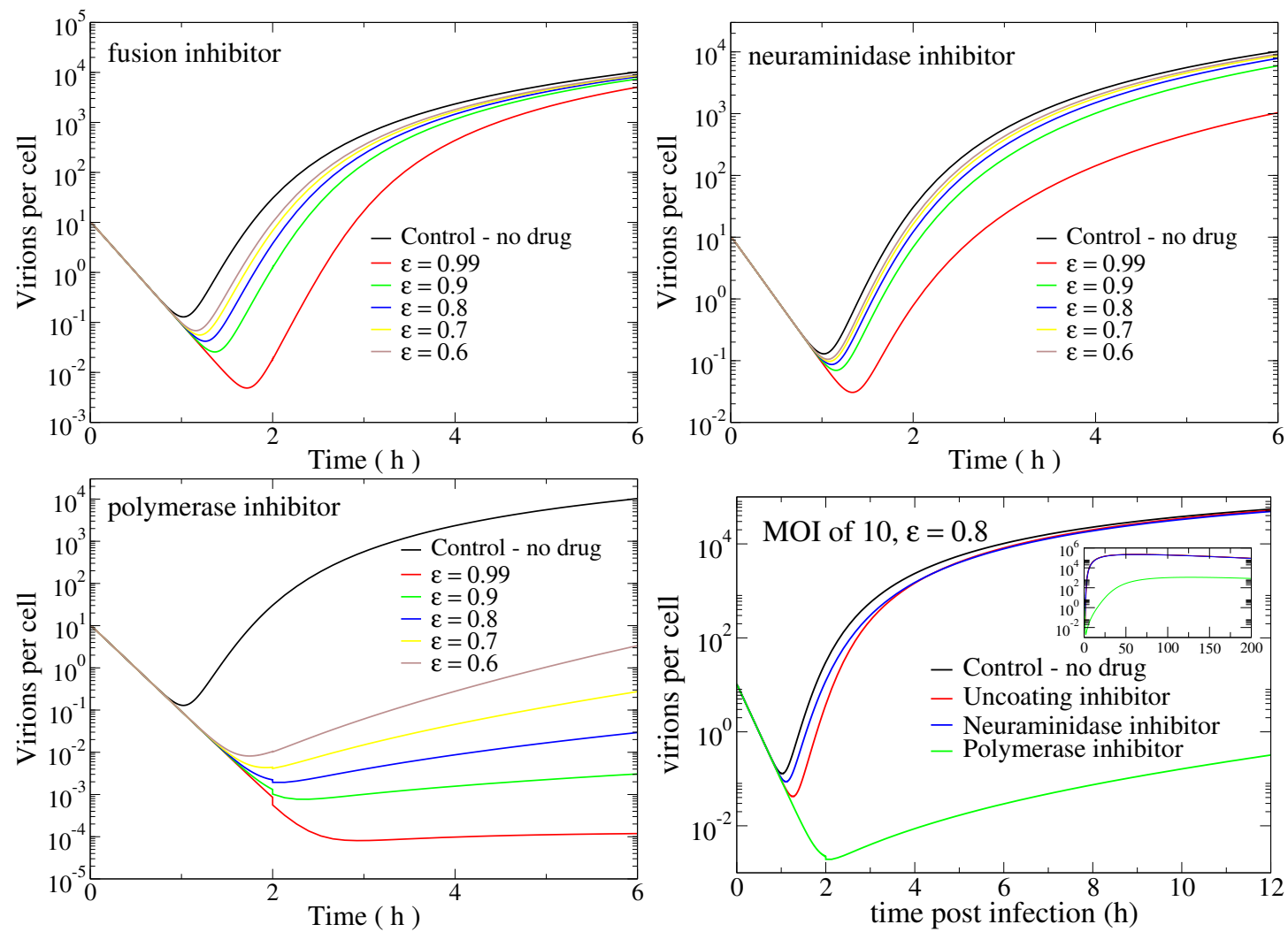

Figure 3.18: Effect of antiviral treatment at various efficacies. Infections were initialized by an MOI of 10 and contrasted against efficacies of $0.6,0.7,0.8$, 0.9 , and 0.99 for various inhibitors.

As we have shown previously, when more virions are present initially, production is hastened, as sufficient protein are available to stabilize cRNA and vRNA. Conversely, production is delayed with lower inocula. Increased efficacy of fusion inhibitors results in a delayed release, similar in nature to an infection with a lower MOI. Assuming a perfect infection where, each virion can lead to an infection, an inoculum of 10 virions per cell with a drug efficacy of $90 \%,(\varepsilon=0.9)$ should result in an infection. Although the MM can predict infection for low MOI experiments, experimentally an infection would not occur for one cell. For example, if the initial 
inoculum was smaller, or the efficacy of the drug increased (to say a $\varepsilon$ of 0.99) then mathematically $1 / 10$ of a virion would produce an infection. Although we can mathematically model this, it is biologically untrue that it would lead to an infection, as this represents less than one segment, from the 8 total segments in IAV, producing an infection. In order to account for this accurately, we would have to use a stochastic MM, rather than a deterministic one. Consistent with our earlier predictions that superinfection with increased inoculum resulted in hastened production, an effective decrease in inoculum results in a delayed production, shown in Figure 3.18.

Neuraminidase can be used as a marker of successful infection as it is trafficked to the cell surface during infection, and is required for the detachment of virions from host membranes. Neuraminidase inhibitors block the active site of neuraminidase which, normally cleaves sialic acid to free progeny virions, thus targeting the release of progeny virions. We found that increased efficacy of a neuraminidase inhibitor results in a delayed release. Progeny virions are not impeded in production but, rather, in their rate of budding, as seen in Figure 3.18. Resistant variants to these inhibitors are limited, as mutations which decrease the effectiveness of the drug also tend to decrease the competitive fitness of the virion [32].

The kinetics of IAV infection with treatment with neuraminidase inhibitors were mathematically modelled by Dobrovolny et al. who found that increasing efficacy of the drug caused a delay and reduction of progeny [54]. Our findings agree with the delay predicted in Dobrovolny et al.'s MM and biological data as neuraminidase inhibitors prevent the virus from budding off. Our MM is dissimilar to experimental data in that the MM predicts progeny virions always reaches the same viral titre in the end, regardless of antiviral efficacy, as opposed experimentally where fewer virions are produced with increased treatment. In our mathematical representation of infection, neuraminidase inhibitors act by blocking the release of nuclear vRNPs via $\mathrm{r}_{\mathrm{vRNP}}^{\text {release }}$, however, do not affect the production of said nuclear vRNPs. Both MMs sustain production until 2.5 days, thus our single cell MM appears to agree with current multicellular MMs. 
Viral polymerase inhibitors are broad range nucleoside analogues for RNA and DNA elongation. They structurally resemble adenosine and guanine and, as such, they get competitively misincorporated by viral RNA dependant viral polymerase, affecting the rate of mRNA, cRNA and vRNA synthesis. Figure 3.18 shows that increased efficacy of polymerase inhibitors results in reduced viral production, with results shown up to 200 hours post infection. With increasing drug concentration fewer progeny virions are produced overall. Unlike fusion inhibitors, which require one successful entry breach in order for the remainder of the replication cycle to proceed as usual, polymerase inhibitors affect all steps.

Increased efficacy of drug utilized during infection resulted in delayed release of progeny, as seen in Figure 3.18. Fusion and neuraminidase inhibitors had no effect on total virions released, while polymerase inhibitors reduced progeny. At an efficacy of $80 \%$ polymerase inhibitors were predicted to have the largest impact. 


\section{Chapter 4}

\section{Discussion}

Our MM is capable of reproducing data for various stages of infection; attachment, fusion, mRNA, vRNA and cRNA, and release of progeny virions. When compared to Heldt et al.'s MM, ours is smaller by 19 variables and 23 parameters. However, it is still capable of reproducing important events. We ensured that the processes represent biological events, and utilized data to validate our MM where available. When a MM works well, it can allow us to predict the course of experiments; where we otherwise lack data, or where measurable output might be below the limit of detection, or where there is a large error in experimental data collection.

As we do not explicitly incorporate DIPs into our current MM, we preformed an imperfect test to evaluate the impact of superinfection with more than one virion. We test how disturbances in infection amounts and timing can change the course of infection, The increased input MOI results in shorter eclipse phase $(\approx 1 \mathrm{~h})$ but no change in the total viral yield. As shown in Figure 3.13 our MM predicts that there is a predetermined amount of progeny virion, and that the addition of more infectious virions allows for earlier production of progeny. It has been shown by Bellett and Cooper and Nayak et al. that an increased concentration of initial inoculum results in a lower virus yield. Superinfection events can result in a reduced amount of standard virus, as progeny DIPs hijack components required for infectious viral particle assembly. DIPs may not be recorded in a plaque assays, as 
they can fail to produce a productive infection [51,52]. Our MM lacks the ability to predict this reduction in virus yield as we do not incorporate DIPs. This is a future point of improvement of the MM, as DIPS have potential use as antiviral agents. Characterizing their influence with MMs could play a critical role, especially as strains of IAV are becoming resistant to commonly used antivirals [55].

We considered infections initialized by a single virion to investigate the length of time after the initial infection where a secondary infection can occur (superinfection window) in our MM, and to what point it can have an effect i.e. how late would a large change, DIP or otherwise, have an impact on infection. The MM does not predict that more virus is made when infected at a higher MOI, but rather that it is made faster. The impact of superinfection decreases as a function of delay: after 2-3 hours it no loner has an impact. This superinfection window has been studied and estimated to be up to $5 \mathrm{~h}$ post initial infection $[51-53,56]$.

Heldt et al.'s MM had programmed shutdown of infection, i.e. cessation of production, due to a massive export of vRNPs from the nucleus, resulting in no genetic material with which to continue replication. Our MM also has a large production of progeny virions, and also ceases production by 72 hours, as seen in Figure 3.15 insert. This means that our MM shuts down without the need for the mathematical incorporation of cellular signals such as ER stress or toxicity. Second pulse of virions cannot spur it into a second round of infection. This is because once there are sufficient progeny vRNP and proteins made, there begins a massive export of template vRNP from the nucleus to the cytoplasm. Template vRNP loss to budding virions results in the production of less mRNA and cRNA, which decay over time. Further adjustments to the MM will have to be preformed to overcome the loss of all nuclear vRNPs. To maintain sufficient nuclear vRNPs, to continue the production of progeny vRNPs, we can impose a duration of time which a vRNP will stay in the nucleus before being exported to the cytoplasm.

In future work, our MM could be adjusted such that cell death will be consistent with known cellular life spans, including cell response such as interferon, and programmed cell death [37]. By scaling our MM to multicellular, and including the addition of cellular regeneration, we may be able to produce a sustained infection 
consistent to that seen in multicellular infections, between $12-14 \mathrm{~h}$ [57]. Lastly the omission of ER stress, toxicity or immune response may cause us to predict a lower total virus yield than currently, as those factors would reduce progeny production. The inclusion of ER stress, toxicity or interferon may cause a higher loss of virions, thus causing the MM to require a higher production to maintain predictions of mRNA, cRNA and vRNA.

There is no 'typical' human influenza A viral infection, due to strains, cell type, and cell-to-cell variation, but we can compare averaged behaviour. With our MM, we can predict the effects of antiviral treatment, causing delay and reduction of viral titre $[37,38]$. Using our MM we can contrast and compare a plethora of results, and use them to compare circulating strains and guide decisions for prescribed antivirals.

Through the use of MMs, Paradis et al. showed that there was a significant lengthening of the eclipse phase between H275Y and I223V substituted H1N1pdm09 strain as compared to the wild-type. Dobrovolny et al. illustrated, using MMs, the effects of antivirals at varying doses when administered at various points during infection with IAV, which had the largest reduction of viral titre when administered early and with high efficacy. Dobrovolny et al. illustrated that normally, treatment with antivirals can be affective if administered within 48 hours of infection, but that for more severe strains of IAV, treatment can still be effective when administered up to 6 days post infection, as high viral titres are sustained longer $[38,54]$. Armed with this, we can forge forward, aiming to determine which antivirals are most effective against new strains, and which antiviral combination therapies will less likely lead to resistance mutation.

We have constructed a MM which captures the intracellular kinetics of IAV replication. Using this MM with experimental data, we can see that although it has fewer parameters and variables compared to Heldt et al.'s model, it still captures the key features of IAV infection. The MM predicts that viral production is hastened with increased inoculum, and forecasts the effect of antivirals with different mode of actions. However, these predictions remain to be further validated (beyond the scope of this thesis). Future projects from this research encompass 
predicting the expected efficacy in vivo as well as the mode of action and efficacy of novel drug compounds. This work will hopefully lead to better understanding of IAV and aid in the development of new antiviral drugs. Such extensions of the present MM may then be used to determine the mode of action of antiviral compounds. 


\section{Bibliography}

[1] Corwin M. Nycholat, Ryan McBride, Damian C. Ekiert, Rui Xu, Janani Rangarajan, Wenjie Peng, Nahid Razi, Michel Gilbert, Warren Wakarchuk, Ian A. Wilson, and James C. Paulson. Recognition of sialylated poly-LacNAc on Nand O-linked glycans by human and avian influenza a virus hemagglutinins. Angew. Chem. Int. Ed. Engl., 51(20):4860-4863, May 2012.

[2] Robert B Couch. Orthomyxoviruses. In Samuel Baron, editor, Medical Microbiology. 4th edition, chapter 58. University of Texas Medical Branch at Galveston, Galveston, Texas, 1996. http://www.ncbi.nlm.nih.gov/books/ NBK8611/.

[3] Song A, Myojo K, Laudenslager J, Harada D, Miura T, Suzuki K, KuniKamochi R, Soloff R, Ohgami K, and Kanda Y. Evaluation of a fully human monoclonal antibody against multiple influenza a viral strains in mice and a pandemic H1N1 strain in nonhuman primates. Antiviral Res., 111:60-68, November 2014.

[4] Public Health Agency of Canada. Influenza. 2016. http://www. phac-aspc. gc.ca/influenza/flu-stat-eng.php.

[5] Catherine A.A. Beauchemin, James J. McSharry, George L. Drusano, Jack T. Nguyen, Gregory T. Went, Ruy M. Ribeiro, , and Alan S. Perelson. Modeling amantadine treatment of inuenza A virus in vitro. J. Virol., 254(2):439-451, July 2008. 
[6] Perelson AS, Neumann AU, Markowitz M, Leonard JM, and Ho DD. HIV1 dynamics in vivo: virion clearance rate, infected cell life-span, and viral generation time. Science, 271(5255):1582-1586, March 1996.

[7] Holder BP, Liao LE, Simon P, Boivin G, and Beauchemin CA. Design considerations in building in silico equivalents of common experimental influenza virus assays. Autoimm., 44(4):282-293, June 2011.

[8] Prasith Baccam, Catherine Beauchemin, Catherine A. Macken, Frederick G. Hayden, , and Alan S. Perelson. Kinetics of influenza A virus infection in humans. J. Virol., 80(15):7590-7599, August 2016.

[9] Frank S. Heldt, Timo Frensing, and Udo Reichl. Modeling the intracellular dynamics of influenza virus replication to understand the control of viral RNA synthesis. J. Virol., 86(15):7806-7817, August 2012.

[10] Sidorenko Y and Reichl U. Structured model of influenza virus replication in MDCK cells. Biotechnol. Bioeng., 88(1):1-14, October 2004.

[11] Nicole M. Bouvier and Peter Palese. The biology of influenza viruses. Vaccine, 26(Suppl 4):D49-D53, September 2008.

[12] Julia Dubois, Olivier Terrier, and Manuel Rosa-Calatrava. Influenza viruses and mRNA splicing: Doing more with less. mBio., 5(3), May 2014.

[13] Patricia Resa-Infante, Núria Jorba, Rocio Coloma, and Juan Ortín. The influenza rna synthesis machine: Advances in its structure and function. RNA Biol., 8(2):207215, March 2011. http://dx.doi.org/10.4161/rna. 8.2.14513.

[14] Donna M. Tscherne and Adolfo García-Sastre. Virulence determinants of pandemic influenza viruses. J. Clin. Invest., 121(1):6-13, January 2011.

[15] Jorba N, Coloma R, and Ortín J. Genetic trans-complementation establishes a new model for influenza virus RNa transcription and replication. PLoS Pathog., 5(5):1-14, May 2009. 
[16] FT Vreede and GG Brownlee. Influenza virion-derived viral ribonucleoproteins synthesize both mrna and crna in vitro. jvi-abb, 81(5):2196-2204, 2007.

[17] Duncan Paterson and Ervin Fodor. Emerging roles for the influenza a virus nuclear export protein (NEP). PLoS Pathog., 8(12):1-8, December 2012.

[18] Lamb RA and Krug RM. Orthomyxoviridae: the viruses and their replication. In Knipe DM and Howley PM, editors, Fields virology, 4th Edition. Lippincott Williams \& Wilkins, Philadelphia, PA, 2001. .

[19] Zheng W and Tao YJ. Structure and assembly of the influenza a virus ribonucleoprotein complex. FEBS Lett., 587(8):1206-1214, April 2013.

[20] Yi-ying Chou, Reza Vafabakhsh, Sultan Doğanay, Qinshan Gao, Taekjip Ha, and Peter Palese. One influenza virus particle packages eight unique viral rnas as shown by fish analysis. Proceedings of the National Academy of Sciences, 109(23):9101-9106, 2012.

[21] Takeshi Noda and Yoshihiro Kawaoka. Packaging of influenza virus genome: robustness of selection. Proceedings of the National Academy of Sciences, 109(23):8797-8798, 2012.

[22] Takeshi Noda, Hiroshi Sagara, Albert Yen, Ayato Takada, Hiroshi Kida, R Holland Cheng, and Yoshihiro Kawaoka. Architecture of ribonucleoprotein complexes in influenza A virus particles. Nature, 439(7075):490-492, 2006.

[23] Centre for Disease Control. Antiviral drug resistance. 2016. http://www . cdc . gov/flu/professionals/antivirals/antiviral-drug-resistance.htm.

[24] Julia Hütter, Jana V Rödig, Dirk Höper, Peter H Seeberger, Udo Reichl, Erdmann Rapp, and Bernd Lepenies. Toward animal cell culture-based influenza vaccine design: Viral hemagglutinin N-glycosylation markedly impacts immunogenicity. The Journal of Immunology, 190(1):220-230, 2013.

[25] Tokiko Watanabe, Maki Kiso, Satoshi Fukuyama, Noriko Nakajima, Masaki Imai, Shinya Yamada, Shin Murakami, Seiya Yamayoshi, Kiyoko IwatsukiHorimoto, Yoshihiro Sakoda, et al. Characterization of h7n9 influenza a viruses isolated from humans. Nature, 501(7468):551-555, 2013. 
[26] Rui Xu, Robert $\mathrm{P}$ de Vries, Xueyong Zhu, Corwin M Nycholat, Ryan McBride, Wenli Yu, James C Paulson, and Ian A Wilson. Preferential recognition of avian-like receptors in human influenza a h7n9 viruses. Science, 342(6163):1230-1235, 2013.

[27] T Stegmann, J G Orsel, J D Jamieson, and P J Padfield. Limitations of the octadecylrhodamine dequenching assay for membrane fusion. Biochemistry, 307(Pt 3):875-878, May 1995.

[28] Shane Crotty, David Maag, Jamie J Arnold, Weidong Zhong, Johnson YN Lau, Zhi Hong, Raul Andino, and Craig E Cameron. The broad-spectrum antiviral ribonucleoside ribavirin is an RNA virus mutagen. Nature medicine, 6(12):1375-1379, 2000.

[29] Kalyan Das, James M Aramini, Li-Chung Ma, Robert M Krug, and Eddy Arnold. Structures of influenza a proteins and insights into antiviral drug targets. Nature structural \& molecular biology, 17(5):530-538, 2010.

[30] Wei-juan $\mathrm{Xu}$, Ning Wei, Yi Xu, and Shao-hua Hu. Does amantadine induce acute psychosis? a case report and literature review. Neuropsychiatric disease and treatment, 12:781, 2016.

[31] Arnold S Monto. Antivirals and influenza: frequency of resistance. The Pediatric infectious disease journal, 27(10):S110-S112, 2008.

[32] Kim JH, Resende R, Wennekes T, Chen HM, Bance N, Buchini S, Watts AG, Pilling P, Streltsov VA, Petric M, Liggins R, Barrett S, McKimmBreschkin JL, Niikura M, and Withers SG. Mechanism-based covalent neuraminidase inhibitors with broad-spectrum influenza antiviral activity. Science, 340(6128):71-75, April 2013.

[33] Yousuke Furuta, Kazumi Takahashi, Masako Kuno-Maekawa, Hidehiro Sangawa, Sayuri Uehara, Kyo Kozaki, Nobuhiko Nomura, Hiroyuki Egawa, and Kimiyasu Shiraki. Mechanism of action of t-705 against influenza virus. Antimicrob. Agents Chemother., 49(3):981-986, March 2005. 
[34] Takashi Nagata, Alan K Lefor, Manabu Hasegawa, and Masami Ishii. Favipiravir: a new medication for the Ebola virus disease pandemic. Disaster medicine and public health preparedness, 9(01):79-81, 2015.

[35] Isabel Nunes-Correia, Jo uo Ramalho-Santos, Shlomo Nir, and Maria C. Pedroso de Lima. Interactions of influenza virus with cultured cells: Detailed kinetic modeling of binding and endocytosis. Biochemistry, 38(3):1095-1101, December 1999.

[36] Eiryo Kawakami, Tokiko Watanabe, Ken Fujii, Hideo Goto, Shinji Watanabe, Takeshi Noda, and Yoshihiro Kawaoka. Strand-specific real-time RT-PCR for distinguishing influenza vrna, crna, and mrna. vm-abb, 173(1):1-6, 2011.

[37] Benjamin P Holder, Philippe Simon, Laura E Liao, Yacine Abed, Xavier Bouhy, Catherine AA Beauchemin, and Guy Boivin. Assessing the in vitro fitness of an oseltamivir-resistant seasonal A/H1N1 influenza strain using a mathematical model. PLoS One, 6(3):e14767, 2011.

[38] Eric G Paradis, Lady Tatiana Pinilla, Benjamin P Holder, Yacine Abed, Guy Boivin, and Catherine AA Beauchemin. Impact of the H275Y and I223V mutations in the neuraminidase of the 2009 pandemic influenza virus in vitro and evaluating experimental reproducibility. PloS one, 10(5):e0126115, 2015.

[39] John W. Eaton, David Bateman, Søren Hauberg, and Rik Wehbring. GNU Octave version 4.0.0 manual: a high-level interactive language for numerical computations. 2016.

[40] Melike Lakadamyali, Michael J Rust, Hazen P Babcock, and Xiaowei Zhuang. Visualizing infection of individual influenza viruses. Proceedings of the $\mathrm{Na}$ tional Academy of Sciences, 100(16):9280-9285, 2003.

[41] Hazen P Babcock, Chen Chen, and Xiaowei Zhuang. Using single-particle tracking to study nuclear trafficking of viral genes. Biophysical journal, 87(4):2749-2758, 2004.

[42] Katharina Ribbeck and Dirk Görlich. Kinetic analysis of translocation through nuclear pore complexes. The EMBO journal, 20(6):1320-1330, 2001. 
[43] Xavier Darzacq, Yaron Shav-Tal, Valeria de Turris, Yehuda Brody, Shailesh M Shenoy, Robert D Phair, and Robert H Singer. In vivo dynamics of rna polymerase ii transcription. Nature structural \& molecular biology, 14(9):796$806,2007$.

[44] Agustín Portela and Paul Digard. The influenza virus nucleoprotein: a multifunctional RNA-binding protein pivotal to virus replication. Journal of general virology, 83(4):723-734, 2002.

[45] Yoav Arava, Yulei Wang, John D Storey, Chih Long Liu, Patrick O Brown, and Daniel Herschlag. Genome-wide analysis of mRNA translation profiles in saccharomyces cerevisiae. Proceedings of the National Academy of Sciences, 100(7):3889-3894, 2003.

[46] Alexander S Spirin. Ribosome structure and protein biosynthesis. Benjamin/Cummings Pub. Co., Advanced Book Program, 1986.

[47] Cheng-Chi Wang, Juine-Ruey Chen, Yung-Chieh Tseng, Che-Hsiung Hsu, YuFu Hung, Shih-Wei Chen, Chin-Mei Chen, Kay-Hooi Khoo, Ting-Jen Cheng, Yih-Shyun E. Cheng, Jia-Tsrong Jan, Chung-Yi Wu, Che Ma, and Chi-Huey Wong. Glycans on influenza hemagglutinin affect receptor binding and immune response. Proc. Natl. Acad. Sci. U.S.A., 106(43):18137-18142, October 2009.

[48] Bruce Alberts, Alexander Johnson, Julian Lewis, Martin Raff, Keith Roberts, and Peter Walter. FIX ME. How cells read the genome: From DNA to protein. In Bruce Alberts, Alexander Johnson, Julian Lewis, Martin Raff, Keith Roberts, and Peter Walter., editors, Molecular Biology of the Cell 4th Ed., chapter 6. Garland Science, New York, NY, 2002. http://www.ncbi.nlm. nih.gov/books/NBK21050/.

[49] Nelson DL, Cox MM, and Lehninger AL FIX ME. Biosynthesis of amino acids, nucleotides, and related molecules. In Nelson DL, Cox MM, and Lehninger AL, editors, Lehninger principles of biochemistry, 3rd Edition. Worth Publishers, New York, NY, 2000. 
[50] Ashley York and Ervin Fodor. Biogenesis, assembly, and export of viral messenger ribonucleoproteins in the influenza a virus infected cell. RNA Biol., 10(8):1274-1282., August 2013.

[51] DP Nayak, K Tobita, J M Janda, A R Davis, and B K De. Homologous interference mediated by defective interfering influenza virus derived from a temperature-sensitive mutant of influenza virus. J. Virol., 28(1):375-386, October 1978.

[52] AJD Bellett and PD Cooper. Some properties of the transmissible interfering component of vesicular stomatitis virus preparations. Microbiology, 21(3):498$509,1959$.

[53] Philip I Marcus, John M Ngunjiri, and Margaret J Sekellick. Dynamics of biologically active subpopulations of influenza virus: plaque-forming, noninfectious cell-killing, and defective interfering particles. jvi-abb, 83(16):8122-8130, 2009.

[54] Dobrovolny HM, Gieschke R, Davies BE, Jumbe NL, and Beauchemin CA. Neuraminidase inhibitors for treatment of human and avian strain influenza: A comparative modeling study. J. Theor. Biol., 269(1):234-244, 2011.

[55] Nigel J Dimmock and Andrew J Easton. Defective interfering influenza virus RNAs: time to reevaluate their clinical potential as broad-spectrum antivirals. Journal of virology, 88(10):5217-5227, 2014.

[56] Laura E. Liao, Shingo Iwami, and Catherine A. A. Beauchemin. (in)validating experimentally-acquired knowledge about influenza a defective interfering particles. Journal of the Royal Society Interface, 2016.

[57] OP Zhirnov, TE Konakova, T Wolff, and H-D Klenk. Ns1 protein of influenza a virus down-regulates apoptosis. Journal of virology, 76(4):1617-1625, 2002. 